Portland State University

PDXScholar

Dissertations and Theses

Dissertations and Theses

Winter 2-22-2013

\title{
Joint Angle Tracking with Inertial Sensors
}

Mahmoud Ahmed El-Gohary

Portland State University

Follow this and additional works at: https://pdxscholar.library.pdx.edu/open_access_etds

Part of the Artificial Intelligence and Robotics Commons, and the Other Electrical and Computer Engineering Commons

Let us know how access to this document benefits you.

\section{Recommended Citation}

El-Gohary, Mahmoud Ahmed, "Joint Angle Tracking with Inertial Sensors" (2013). Dissertations and Theses. Paper 661.

https://doi.org/10.15760/etd.661

This Dissertation is brought to you for free and open access. It has been accepted for inclusion in Dissertations and Theses by an authorized administrator of PDXScholar. Please contact us if we can make this document more accessible: pdxscholar@pdx.edu. 
Joint Angle Tracking with Inertial Sensors

by

Mahmoud Ahmed El-Gohary

A dissertation submitted in partial fulfillment of the requirements for the degree of

Doctor of Philosophy

in

Electrical and Computer Engineering

\author{
Dissertation Committee: \\ James McNames, Chair \\ Dan Hammerstrom \\ Gerardo Lafferriere \\ Andres La Rosa \\ Martin Siderius
}

Portland State University

2013 


\section{Abstract}

The need to characterize normal and pathological human movement has consistently driven researchers to develop new tracking devices and to improve movement analysis systems. Movement has traditionally been captured by either optical, magnetic, mechanical, structured light, or acoustic systems. All of these systems have inherent limitations. Optical systems are costly, require fixed cameras in a controlled environment, and suffer from problems of occlusion. Similarly, acoustic and structured light systems suffer from the occlusion problem. Magnetic and radio frequency systems suffer from electromagnetic disturbances, noise and multipath problems. Mechanical systems have physical constraints that limit the natural body movement.

Recently, the availability of low-cost wearable inertial sensors containing accelerometers, gyroscopes, and magnetometers has provided an alternative means to overcome the limitations of other motion capture systems. Inertial sensors can be used to track human movement in and outside of a laboratory, cannot be occluded, and are low cost. To calculate changes in orientation, researchers often integrate the angular velocity. However, a relatively small error or drift in the measured angular velocity leads to large integration errors. This restricts the time of accurate measurement and tracking to a few seconds. To compensate that drift, complementary data from accelerometers and magnetometers are normally integrated in tracking systems that utilize the Kalman filter (KF) or the extended Kalman filter (EKF) to fuse the nonlinear inertial data. Orientation estimates are only accurate for brief moments when the body is not moving and acceleration is only due to gravity. Moreover, suc- 
cess of using magnetometers to compensate drift about the vertical axis is limited by magnetic field disturbance.

We combine kinematic models designed for control of robotic arms with state space methods to estimate angles of the human shoulder and elbow using two wireless wearable inertial measurement units. The same method can be used to track movement of other joints using a minimal sensor configuration with one sensor on each segment. Each limb is modeled as one kinematic chain. Velocity and acceleration are recursively tracked and propagated from one limb segment to another using Newton-Euler equations implemented in state space form. To mitigate the effect of sensor drift on the tracking accuracy, our system incorporates natural physical constraints on the range of motion for each joint, models gyroscope and accelerometer random drift, and uses zero-velocity updates. The combined effect of imposing physical constraints on state estimates and modeling the sensor random drift results in superior joint angles estimates. The tracker utilizes the unscented Kalman filter (UKF) which is an improvement to the EKF. This removes the need for linearization of the system equations which introduces tracking errors.

We validate the performance of the inertial tracking system over long durations of slow, normal, and fast movements. Joint angles obtained from our inertial tracker are compared to those obtained from an optical tracking system and a high-precision industrial robot arm. Results show an excellent agreement between joint angles estimated by the inertial tracker and those obtained from the two reference systems. 


\section{Dedication}

For my mother Zahira \& my father Ahmed;

my sisters Gamila \& Fatma; my brothers Ali, Mohamad \& Khalid; and for Greer. 


\section{Acknowledgements}

I would like to express my gratitude to the many people who made working on my Ph.D. a wonderful experience. Foremost, I am deeply indebted to my advisor Dr. James McNames. It has been a real pleasure working with you. You have guided and supported me. You expected great things from me with such sheer confidence, that I eventually felt that same confidence in myself. I admire your hard work and high academic standards, which have set an example of excellence for all your students and myself. I continue to be inspired to meet a high level of achievement, a standard you modeled for me. Above all, you have become a true friend, thank you.

During this work, I have collaborated with many colleagues for whom I have great regard. I wish to extend my warmest thanks to all those who have helped me with my work in the Biomedical Signal Processing Laboratory at PSU, and in the Balance Disorders Laboratory at OHSU.

I owe my most sincere gratitude to Greer Carver for her love and encouragement. Thank you for your support when I have needed it the most. To quote one of your favorite poets, Khalil Gibran"1, "And think not you can direct the course of love, for love, if it finds you worthy, directs your course." Your love and friendship have changed the course of my life beyond my wildest dreams.

Finally, I would like to thank my family for their infinite support throughout my life. Their unconditional love and understanding has encouraged me to work hard and value education; to capitalize on the gifts given to me. My mother's firm and kind-

\footnotetext{
${ }^{1}$ The Prophet, by Kahlil Gibran
} 
hearted personality taught me to be faithful to my dreams and never bend to difficulty. My father's gentle example taught me that humility is grace and compassion is power. Though my mother and father are no longer here in the flesh, I feel their love still, and their sage advice is no more than a memory away. Thank you my brothers and sisters, with you I discovered the joy that radiates from life and love that endures. Because of you, no matter how far away I may be, I am able to carry home with me. 


\section{Table of Contents}

$\begin{array}{lll}\text { Abstract } & \text { i }\end{array}$

Dedication

Acknowledgements iv

List of Tables $\quad$ ix

List of Figures $\quad$ xi

1 Introduction 1

1.1 Biomedical Applications . . . . . . . . . . . . . 2

1.2 Sports and Virtual Reality Applications . . . . . . . . . . . 5

1.3 Motion Capture Technologies . . . . . . . . . . . . . . . . 6

1.4 Inertial Measurement Unit . . . . . . . . . . . . . . . 9

1.5 Objectives and Significance . . . . . . . . . . . . . 10

1.6 Dissertation Overview . . . . . . . . . . . . . . 12

2 Literature Review $\quad 14$

2.1 Inertial Sensors Algorithms . . . . . . . . . . . . . . 14

2.2 Limitations of Previous Studies . . . . . . . . . . . . . . 20

2.3 Addressing The Limitations . . . . . . . . . . . . . . . 21 
3 Kinematics of Human Motion 23

3.1 Basic Movement Description . . . . . . . . . . . . 23

3.2 Kinematic Chains and Model Development . . . . . . . . 25

3.2.1 Denavit-Hartenberg Convention .......... 26

3.2.2 Denavit-Hartenberg Parameters . . . . . . . . . 28

3.2.3 Link Transformations . . . . . . . . . . . . . 30

3.3 Velocity and Acceleration Propagation . . . . . . . . . . . 31

3.3.1 Netwon-Euler Equations . . . . . . . . . . . . 32

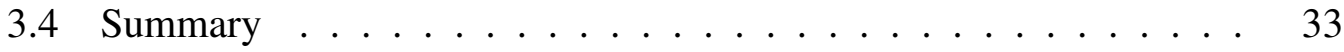

4 Joint Angles Estimation $\quad 34$

4.1 Estimation and Filtering . . . . . . . . . . . . 34

4.2 State Space Models . . . . . . . . . . . . . . . . . . . 36

4.2.1 State Model . . . . . . . . . . . . . . 37

4.2.2 Observation Model . . . . . . . . . . . . . . 38

4.2.3 Nonlinear State Estimator . . . . . . . . . . . . 38

4.3 Model Validation Using Synthetic Data . . . . . . . . . . . . . . . 39

4.3.1 Elbow and Forearm Model . . . . . . . . . . . . . . . 40

4.3.2 Shoulder Model . . . . . . . . . . . . . . . . . . . . 41

4.4 Results .......................... 41

4.5 Summary ........................... 45

5 Performance Assessment 46

5.1 Study Protocol . . . . . . . . . . . . . . . . . 46

5.1.1 Data Collection ................ 48

5.2 Shoulder and Elbow Measurement Model . . . . . . . . . . . . 49

5.3 Results ......................... 52

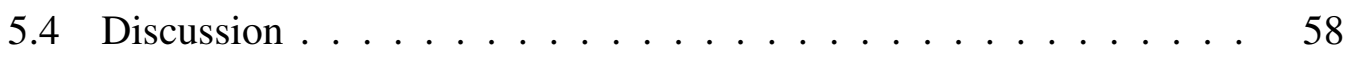


6 Modified Arm Model 61

6.1 Robot Arm Joint Angles . . . . . . . . . . . . . . . . 62

6.1.1 Baseline Performance Results ........... 65

6.2 Modeling Sensor Random Drift . . . . . . . . . . . . . . . . 74

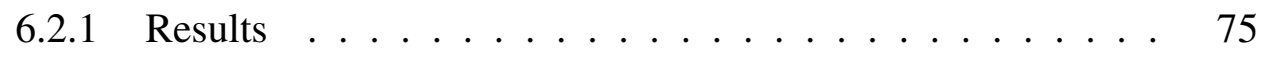

6.3 Anatomical Constraints in Shoulder and Elbow . . . . . . . . . 77

6.3 .1 Results .................... 78

6.4 Zero-Velocity Updates . . . . . . . . . . . . . . . 79

6.4 .1 Results .................... 80

6.5 Modified Model with Bias, Constraints, and Zero-Velocity Updates . 80

6.5.1 Results .................. 81

6.6 Discussion . . . . . . . . . . . . . . . . . 84

7 Nonlinear State Estimation $\quad 87$

7.1 Extended Kalman Filter . . . . . . . . . . . . . . . . . . 88

7.1.1 Extended Kalman Filter Recursions . . . . . . . . . . . . 89

7.2 Unscented Kalman Filter . . . . . . . . . . . . . . . . . . . . 90

7.2.1 Unscented Kalman Filter Recursions . . . . . . . . . . . . . 90

7.3 Particle Filters . . . . . . . . . . . . . . . . . 92

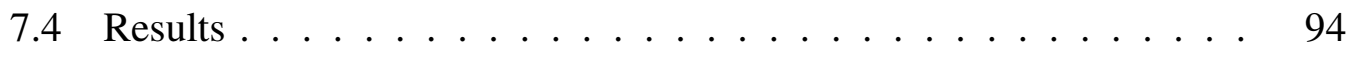

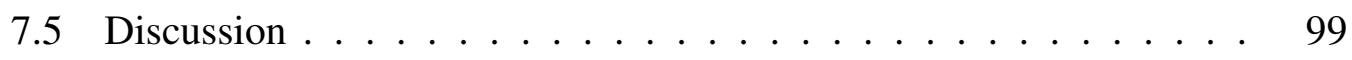

8 Summary And Conclusion $\quad 101$

8.1 Summary ............................. 101

8.2 Contributions ......................... 104

8.3 Conclusion ......................... 107

$\begin{array}{lr}\text { References } & 108\end{array}$ 


\section{List of Tables}

3.1 Denavit-Hartenberg parameters for the shoulder and arm model. The parameter $l_{\mathrm{u}}$ is upper arm length and $l_{\mathrm{f}}$ is the forearm length. The parameters $a_{i}, \alpha_{i}$ and $d_{i}$ are constant, while $\theta_{i}$ is the joint variable that changes when link $i$ rotates with respect to link $i-1$. . . . . . . . 30

4.1 User-specified parameters and sample rate for the unscented Kalman filter-based joint angle tracker. $I$ represents an identity matrix. . . . . .

4.2 Average correlation $r$, and root mean squared error RMSE between true and estimated arm angles. . . . . . . . . . . . . . .

5.1 Average correlation $r$, RMSE, and peak-to-peak error between optical and inertial angles of shoulder and elbow joints. . . . . . . . . . 52

5.2 Average correlation $r$, average RMSE and peak-to-peak average error between optical and inertial estimates of shoulder, elbow and forearm angles during prescribed activities at normal movement rate. . . . . .

6.1 Maximum operating speed and motion range for the robot arm. . . . . 63

6.2 Denavit-Hartenberg parameters for the 6 DOFs arm model. . . . . . . . 64

6.3 Average rotational rate, correlation $r$, and RMSE in angle estimates. . . 65

6.4 RMSE between inertial and true robot angles during medium and fast

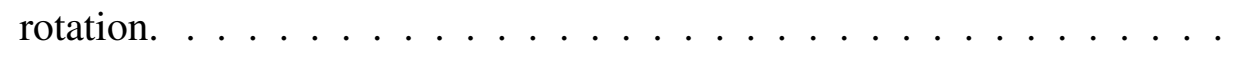


6.5 RMSE of inertial angle estimates using the modified dynamic equations with bias estimates, compared to baseline results of angles during slow

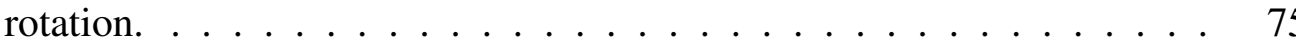

6.6 RMSE of inertial angle estimates using algorithm with physical constraints compared to baseline results during slow rotation. . . . . . . . . 78

6.7 RMSE of inertial angle estimates using zero-velocity updates compared to baseline results during slow rotation. . . . . . . . . . . . . 80

6.8 RMSE of inertial angle estimates with modified arm model compared to baseline results during medium-speed arm rotation. . . . . . . . . . .

7.1 User-specified parameters and sample rate used with the three filter. I represents an identity matrix. . . . . . . . . . . . . . . 94

7.2 Root mean squared error (RMSE) in degrees between true robot arm angles and estimates using the EKF, UKF and PF with 500 and 2500

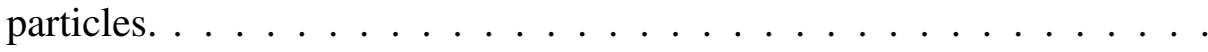




\section{List of Figures}

1.1 Optical motion systems utilize digital cameras to capture markers attached to different body segments. During his performance in "The Polar Express" movie, Tom Hanks wore 152 markers on his face and 48 body markers. The body and facial motion were captured by 72 cameras [71].

1.2 Example of mechanical sensor device (Gypsy). An electromechanical suit manufactured by Animazoo that contains a combination of 37 potentiometers and 2 gyroscopes. Published with permission from Animazoo UK Ltd, Brighton, UK. . . . . . . . . . . . . . . . . . . .

1.3 Opal Inertial measurement units used in this research (APDM Inc., Portland, Oregon, USA). Each unit contains triaxial accelerometers, gyroscopes, and magnetometers. . . . . . . . . . . . . . .

1.4 Reflective markers and Opal inertial sensors placement on the arm of one of the subjects. . . . . . . . . . . . . .

3.1 The arm is modeled with 5 degrees of freedom. Three at the shoulder to allow for flexion/extension, abduction/adduction and internal/external rotation. The fourth allows elbow flexion/extension, and the last allows forearm pronation/supination. . . . . . . . . . . . 
3.2 Diagram of the arm model with Frame 0 as the static reference frame. Frames 1 through 3 represent shoulder flexion/extension, abduction/adduction and internal/external rotation, respectively. Frames 4 through 5 represent the elbow flexion/extension and forearm pronation/supination. . . . .

3.3 The kinematic function of a link is to maintain a fixed relationship between the two joint axes. Each link is characterized by two constants. The link length $a_{i}$ is the distance from $Z_{i}$ to $Z_{i+1}$ measured along their common normal $X_{i}$. The link twist $\alpha_{i}$ is the angle from $Z_{i}$ to $Z_{i+1}$ measured about the $X_{i}$ axis. $\ldots \ldots \ldots \ldots \ldots$

3.4 Neighboring links have a common joint axis $Z$. One parameter that defines the distance along this axis is called the link offset $d_{i}$, the distance from $X_{i-1}$ to $X_{i}$ measured along the $Z_{i}$ axis. The amount of rotation measured about the $Z_{i}$ axis is called joint angle $\theta_{i}$ and it is the angle from $X_{i-1}$ to $X_{i} \ldots \ldots \ldots \ldots \ldots \ldots$

4.1 Synthetic elbow and forearm angles (solid lines), and their estimates

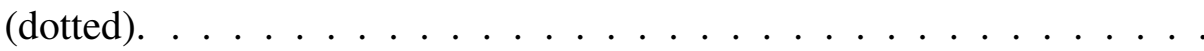

4.2 Error between true and estimated elbow flexion/extension angles (top) with $\mathrm{RMSE}=0.2^{\circ}$, and forearm supination/pronation (bottom) with

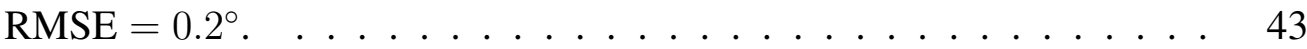

4.3 Synthetic shoulder angles (solid lines), and their estimates (dotted). . . .

4.4 Angle estimation error of shoulder internal/external with $\mathrm{RMSE}=0.2^{\circ}$, adduction/abduction with $\mathrm{RMSE}=0.2^{\circ}$, and flexion/extension with $\mathrm{RMSE}=0.3^{\circ} \ldots \ldots \ldots \ldots \ldots \ldots$

5.1 Reflective markers and Opal inertial measurement units placement on the upper arm and forearm of one of the subjects. . . . . . . . . . . 
5.2 Elbow and forearm angle estimates by the optical system (dashed red line) compared to inertial angles estimate (solid blue line). . . . . . . 53

5.3 Difference between estimates using optical and inertial trackers. . . . . 53

5.4 Shoulder angle estimates by the optical system (dashed red line) compared to inertial angles estimate (solid blue line). . . . . . . . . . 54

5.5 Difference between estimates using optical and inertial trackers. . . . . 54

5.6 Elbow (top) and shoulder (bottom) flexion/extension angle estimates while subject repeatedly extends arm, then touches the nose with the index finger. . . . . . . . . . . . . . . . 56

5.7 Estimate errors of Elbow (top) and shoulder (bottom) flexion/extension. 56

5.8 Shoulder flexion/extension and abduction/adduction angles during fast movement. Inertial (solid blue line) and optical angle estimates (dashed red line). . . . . . . . . . . . . . . . . 57

5.9 Difference between estimates using optical and inertial trackers. . . . . 57

6.1 Opal inertial sensors placement on Epson C3 robot arm. . . . . . . . . . 63

6.2 Diagram of the arm model with Frame 0 as the static reference at the base. Frames 1 and 2 represent shoulder internal/external rotation, and flexion/extension. Frame 3 represents elbow flexion/extension. Frame 4 represents forearm pronation/supination. Wrist flexion/extension, and wrist twist are represented by frames 5 and 6 respectively. . . . . . . .

6.3 Rotational rate data during the last minute from the gyroscope sensor unit placed on the wrist. . . . . . . . . . . . . 66

6.4 Acceleration data during the last minute from the sensor unit placed on the wrist. . . . . . . . . . . . . . . . . 67

6.5 Wrist angles during the last 2 minutes at an average rotation rate of $160 \%$ s. 68

6.6 Elbow angles during the last 2 minutes at an average rotation rate of

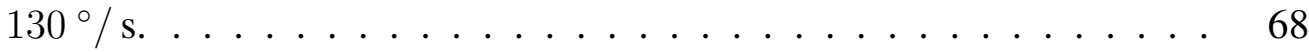


6.7 Wrist joint angle error between true and inertial estimates. . . . . . . . 69

6.8 Elbow joint angle error between true and inertial estimates. . . . . . . 69

6.9 Shoulder angles during the first 2 minutes at an average rotation rate of $112 \%$. . . . . . . . . . . . . . . . 70

6.10 Shoulder angles during the last 2 minutes at an average rotation rate of $112 \%$ s............................ 70

6.11 Wrist angles during the last 2 minutes at an average rotation rate of $320 \% \mathrm{~s} .72$

6.12 Elbow angles during the last 2 minutes at an average rotation rate of $260 \%$. . . . . . . . . . . . . . . . 72

6.13 Shoulder angles during the first 2 minutes at a rotation rate of $225^{\circ} / \mathrm{s}$. 73

6.14 Shoulder angles during the last 2 minutes at a rotation rate of $225^{\circ} / \mathrm{s}$. 73

6.15 Shoulder angles during the last two minutes at a rotation rate of $112 \%$ s. 76

6.16 Shoulder angle error between true and estimates during the last 2 minutes. 76

6.17 Wrist angles during the last 2 minutes at an average rotation rate of $318^{\circ} / \mathrm{s} .82$

6.18 Elbow angles during the last 2 minutes at an average rotation rate of

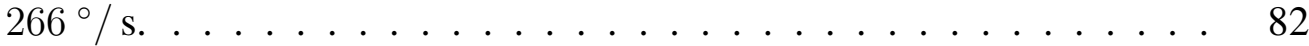

6.19 Shoulder angles of the first 2 minutes at an average rotation rate of $225^{\circ} / \mathrm{s} .83$

6.20 Shoulder angles of the last 2 minutes at an average rotation rate of $225^{\circ} / \mathrm{s} .83$

7.1 True robot shoulder rotation and flexion, and their estimates using UKF. 95

7.2 Error between true robot shoulder angles and estimates using EKF, UKF

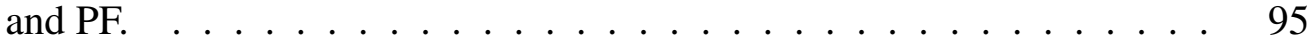

7.3 True robot elbow flexion and supination, and their estimates using UKF. 96

7.4 Error between true robot elbow angles and estimates using EKF, UKF

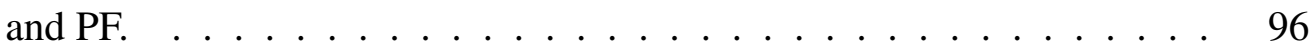

7.5 True robot wrist flexion and supination, and their estimates using UKF. 97

7.6 Error between true robot wrist angles and estimates using EKF, UKF and

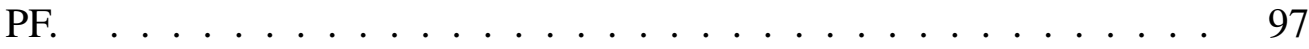




\section{Introduction}

The need to characterize normal and pathological human movement has consistently driven researchers to develop new tracking devices and to improve movement analysis systems. Interest in tracking human movement has been motivated by the wide array of its applications. Research groups from various disciplines study human movement to better understand human motor function, to develop treatment methods aimed at strengthening the mobility of individuals with motor functional limitations, and to develop techniques for improving athletes' performance. Movement has normally been captured by either optical, mechanical, magnetic, acoustic, or inertial sensing systems. Although each of these systems has its own advantages, they all suffer from various limitations. The focus of this research is primarily on using inertial sensing systems to track human motion. More specifically, we use wireless inertial measurement units to track human joint angles.

This chapter discusses some of the common applications of movement tracking in Section 1.1 and Section 1.2. Section 1.3 introduces some of the major motion capture technologies, and discusses their advantages and limitations. The objective and significance of this research are discussed in Section 1.5, and an overview of the rest of the dissertation is presented in Section 1.6. 


\subsection{Biomedical Applications}

Human movement tracking has many applications in the medical field including diagnosis of neurological movement disorders, assessment of treatment, as well as rehabilitation from injury that may have compromised an individual's mobility. To assess the disorder and the degree of motor impairment, clinicians frequently use various clinical rating scales [88]. However, these rating scales are subjective, momentary, coarse and fail to capture subtle changes in patients' motor state which varies considerably and continuously throughout the day $[60,79]$. On the other hand, continuous and objective measurements of a patient's daily physical activities can be used to study the degree of motor impairment, activity fluctuation during the day, and to assess the effect of different treatments and therapies. These measurements can also be used in rehabilitation to help restore motor functions of individuals who suffer from stroke or traumatic brain injury [131].

Among the common neurological problems that affect human motor functions are Parkinson's disease (PD), essential tremor (ET), Huntington's disease (HD), multiple sclerosis (MS), stroke, traumatic brain injury, and spinal cord injuries.

The incidence of Parkinson's disease is about $0.3 \%$ in the United State's population [92] and is approximately $1 \%$ in individuals over the age of sixty [107]. According to the Parkinson's Disease Foundation, 60, 000 Americans are newly diagnosed with PD each year. Cardinal motor symptoms of PD include rigidity and freezing in place, slowed movements (bradykinesia), shaking in an arm or leg when it is not being moved (rest tremor), small shuffling steps (gait), as well as postural instability [69]. Levodopa is the primary drug treatment for PD, however its long-term use is limited by motor complications and involuntary movements known as Levodopainduced dyskinesia [93]. There is no specific laboratory blood analysis or marker for PD, and diagnosis is performed by clinicians with a standard neurological examination. This involves various simple tests of reactions, reflexes and movements, 
as well as mental tasks. Clinicians use rating scales such as the Unified Parkinsons Disease Rating Scale (UPDRS) [37] to score and follow the progression of the disease. Several studies have recently shown that some motor symptoms of PD can be measured with inertial sensors during clinical assessments with prescribed activities [115, 32, 106, 105]. El-Gohary et al. showed that wearable inertial sensors can be used to develop metrics for objective measurements of tremor [34] and of Levodopa-induced dyskinesia in subjects with PD [21]. Zwartjes et al. developed an inertial ambulatory monitoring system that provides a complete motor assessment of tremor, bradykinesia, and hypokinesia [134]. Huang et al. used inertial sensors on the arms of PD patients to measure arm swing. They reported that subjects exhibit asymmetric and reduced arm swing, and that asymmetric arm swing can easily be detected early in the disease's progression [61].

Essential tremor is another common neurological disorder among adults and is estimated to affect up to $4 \%$ of the population [12]. It is characterized by an involuntary and rhythmic tremor of patients' limbs, most typically the hands and arms [30]. Elble et al. successfully used inertial measurement units with a triaxial accelerometer to measure wrist tremor in patients with ET [35]. Badke studied the effects of changing head position and orientation on head tremor in subjects with ET using angular velocity [9].

The frequency of Huntington's disease varies among different populations ranging from an estimated 4 to 10 individuals per 100,000 [62]. Major symptoms include involuntary and jerky movements that may affect the arms, legs or trunk. Other motor disturbances include repetitive and sustained muscle contractions and abnormal movement patterns which impair initiation and execution of movements [104]. Delval et al. used video motion analysis to study gait disturbance in subjects with HD, and observed a clear decrease in gait speed, an increase in stride time [28], and a reduction in the range of joint angles [29]. 
Stroke is a leading cause of motor impairment particularly in the elderly population [85]. More than 1,100,000 Americans have reported difficulties with motor functional limitations following a stroke $[59,49]$. Therapies developed for motor rehabilitation after a stroke require lengthy and repetitive training to promote motor relearning and recovery $[18,41,99]$. Abdullah et al. demonstrated that monitoring position and orientation of upper limb movement during robot-assisted therapy could be used to help therapists quantify the resistance force at each joint and the trajectory of each prescribed rehabilitation exercise [1].

Multiple Sclerosis is a chronic disabling disease that affects the central nervous system. Studies estimated that 400,000 patients suffered from MS in North America, and approximately 1.1 million people worldwide in 2007 [53]. Balance and gait limitations are some of the common symptoms of MS. Spain et al. used body-worn inertial sensors to detect differences in balance and gait between individuals with MS, with healthy controls [112]. The study showed that during gait, MS patients had greater trunk angular range of motion. The study also showed that individuals with MS had significantly greater sway during quiet stance.

Movement capture and analysis has also been used to detect body posture and postural instability in elderly subjects. The older population, persons 65 years or older, represented $13.1 \%$ of the US population in the year 2010 and are expected to grow to be $19 \%$ of the population by 2030 [43]. Hayes et al. used inertial sensors to study limb motions and joint kinematics during swing phase of human gait [56]. Periods of sitting, standing, lying, and walking can be used to evaluate the quality of life of elderly and subjects with chronic diseases such as arthritis and cardiovascular diseases [87]. 


\subsection{Sports and Virtual Reality Applications}

Among other applications, movement analysis has become a standard tool used in professional sports to monitor, analyze and improve athletic performance by recognizing and fine-tuning the quality of the athlete's movement. Most actions during running, kicking, jumping or throwing are rotational and involve the hip, knee, ankle, shoulder, elbow and wrist joints. Wright $e t$ al. used goniometers to measure elbow range of motion including flexion, extension, supination, and pronation in professional baseball pitchers [123]. In his thesis, Lapinski successfully used wearable sensors to capture physical activities during athletic performance [72].

In-depth analysis of the changes in joint angles with changes in athlete posture may be useful for fine-tuning athlete specific techniques to improve performance $[68,13]$ and reduce potential injuries [84]. The key to success of comprehensive analysis is the ability to capture greatest to smallest movement executions over multiple degrees of freedom [16]. Werner et al. examined the relationship between elbow stress in professional baseball pitchers and the kinematic parameters of pitching mechanics [118]. They concluded that $97 \%$ of the variance in elbow stress was related to factors that depend on shoulder abduction angle and angular velocity, shoulder external rotation torque and elbow flexion angle. By optimizing these variables, clinicians and coaches could help reduce athletes' elbow stress and shoulder injuries.

In virtual reality applications, computer graphics systems use movement analysis to provide position and orientation control in a head-mounted display [36, 4, 117]. Heinz et al. used wearable inertial sensors for real-time task recognition in gaming applications [57]. 


\subsection{Motion Capture Technologies}

The primary challenge in human movement tracking and analysis is to design devices and algorithms that can accurately monitor movement regardless of the activity. Human movement can be measured using a wide variety of techniques and sensors including optical, mechanical, magnetic, acoustic, or wearable inertial sensing systems. The measured data is then processed using tracking algorithms either on-line or off-line to estimate the body kinematics.

Optical systems rely on measurements of reflected or emitted light. Motion is captured by placing active or passive reflective markers on the body. Cameras are used to record the markers' positions, and different algorithms are used to compute the position and orientation of the body [101]. Optical systems are the most common and accurate in tracking movement. However, all optical systems suffer from a major disadvantage: there must be a clear line of sight between the source and the sensor. The systems are costly, can only be used in a laboratory environment, and are susceptible to skin movement artifact, all of which limit their usage [117].

Mechanical motion capture systems typically involve an articulated series of metal or plastic pieces linked together with electromechanical transducers such as potentiometers. As the individual moves, the articulated mechanical parts change shape causing the the transducers to move. Mechanical sensors can provide very precise and accurate pose estimates free of occlusion for a single joint. However, they can be cumbersome, hard to use for monitoring daily activities, and can only allow limited range of motion.

Magnetic motion capture systems utilize sensors placed on the body to measure the magnetic fields generated by magnetometers or current induced in an electromagnetic coil when a changing magnetic field passes through the coil [89]. Three orthogonally oriented magnetic sensors in a single sensor unit can provide a 3D vector indicating the units orientation with respect to excitation [91]. Besides their range 


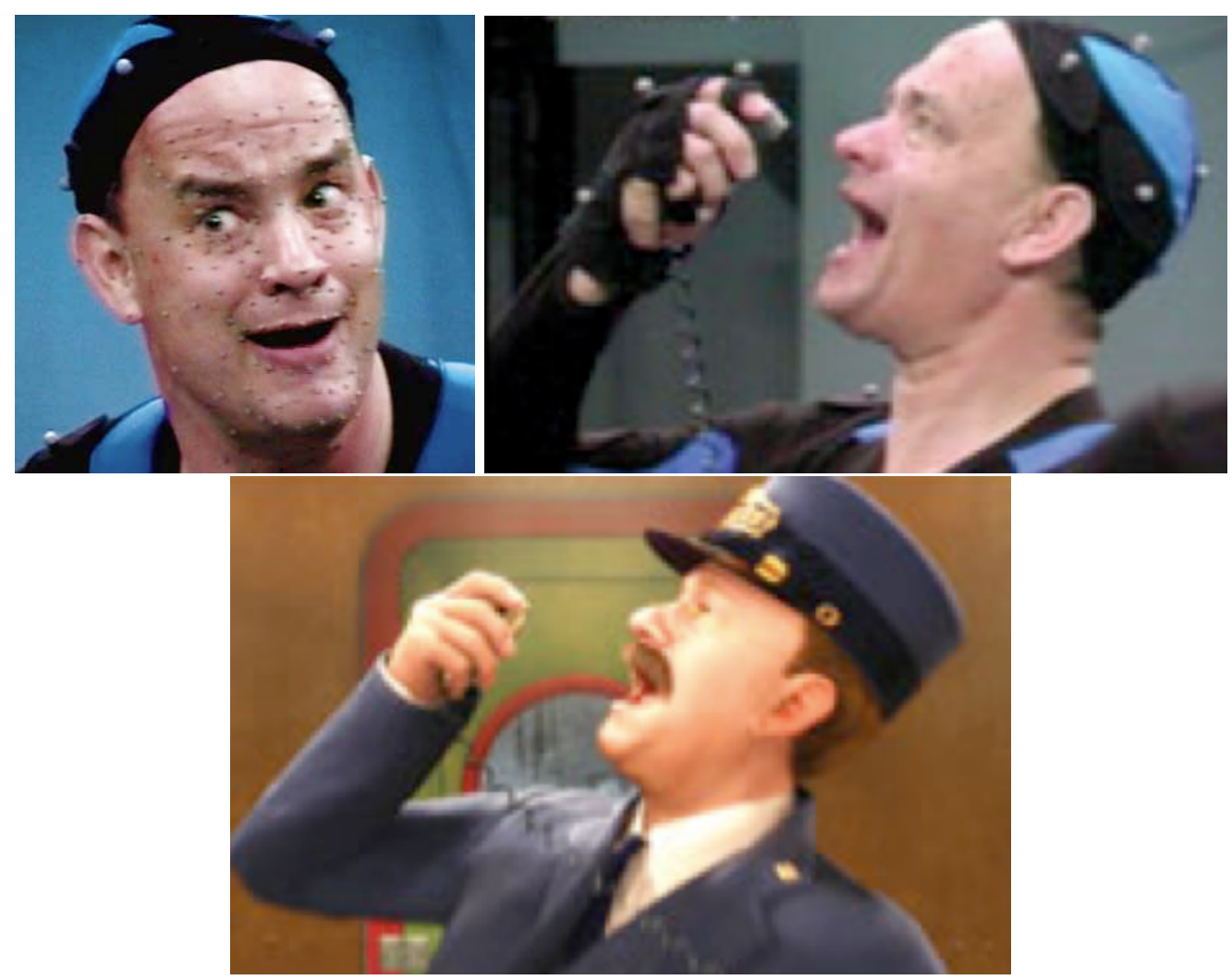

Figure 1.1: Optical motion systems utilize digital cameras to capture markers attached to different body segments. During his performance in "The Polar Express" movie, Tom Hanks wore 152 markers on his face and 48 body markers. The body and facial motion were captured by 72 cameras [71].

limitations, magnetic systems have negative reaction to metal or magnetic fields in the environment. The most common approach to addressing these distortions is to ensure that the surroundings contain no objects that might cause magnetic disturbance [124]. This limits their use to restricted areas where disturbances can be eliminated.

Acoustic motion capture systems are often used in robots for navigation and obstacle avoidance [125]. The systems use emitters and receivers of sound waves [58]. The emitters send out ultrasonic pulses and the delay it takes for these pulses to reach the receivers is used to calculate the distance between the emitter and the receiver. 

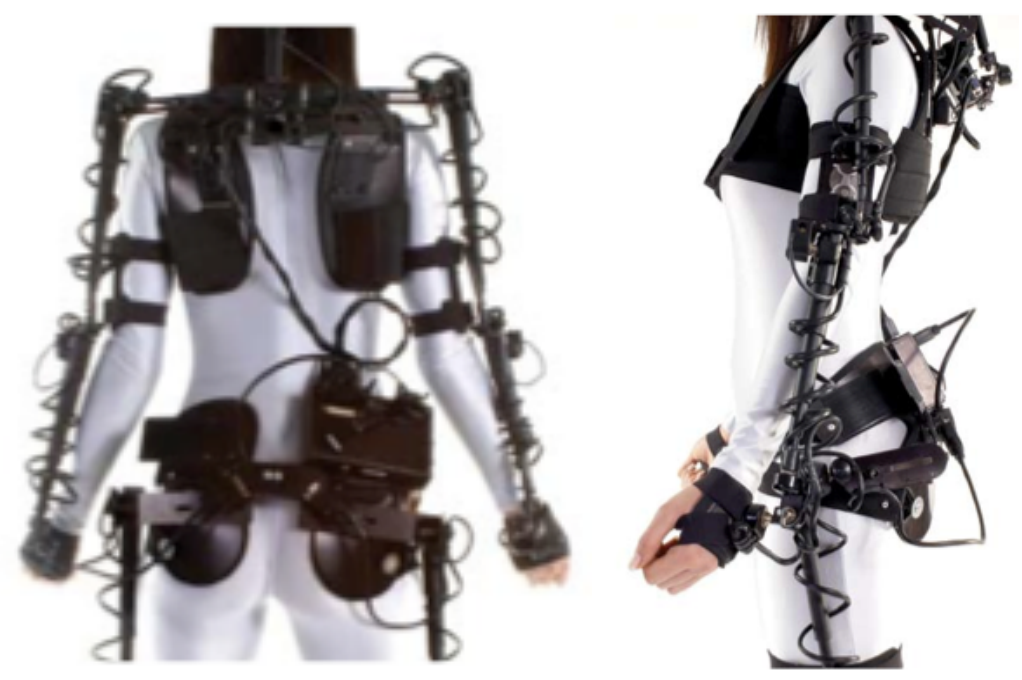

Figure 1.2: Example of mechanical sensor device (Gypsy). An electromechanical suit manufactured by Animazoo that contains a combination of 37 potentiometers and 2 gyroscopes. Published with permission from Animazoo UK Ltd, Brighton, UK.

Acoustic systems require a line of sight between the emitters and the receivers, and are sensitive to perturbations such as humidity, temperature changes and wind.

Inertial measurement systems continue to change rapidly and there have been significant advances in reducing their cost and size in the last few years [11]. Inertial sensors have been widely used to overcome the limitations of other motion capture systems described above. Inertial sensors include, but are not limited to, accelerometers which are used to measure the translational acceleration in addition to gravity, and gyroscopes to measure angular velocities. The sensors can be attached to various body segments and can be used in and outside of a laboratory. Detected movement signals can be continuously recorded while subjects perform daily activities either in the clinic or at home over an extended period of time. Inertial measurement units offer practical and relatively low-cost systems for data acquisition. Collected data can be processed off-line or during real-time movement. More importantly, rapid advance in measurement units and tracking algorithms will soon enable individuals to quantify their own movement, and to receive feedback to assess their improvement 


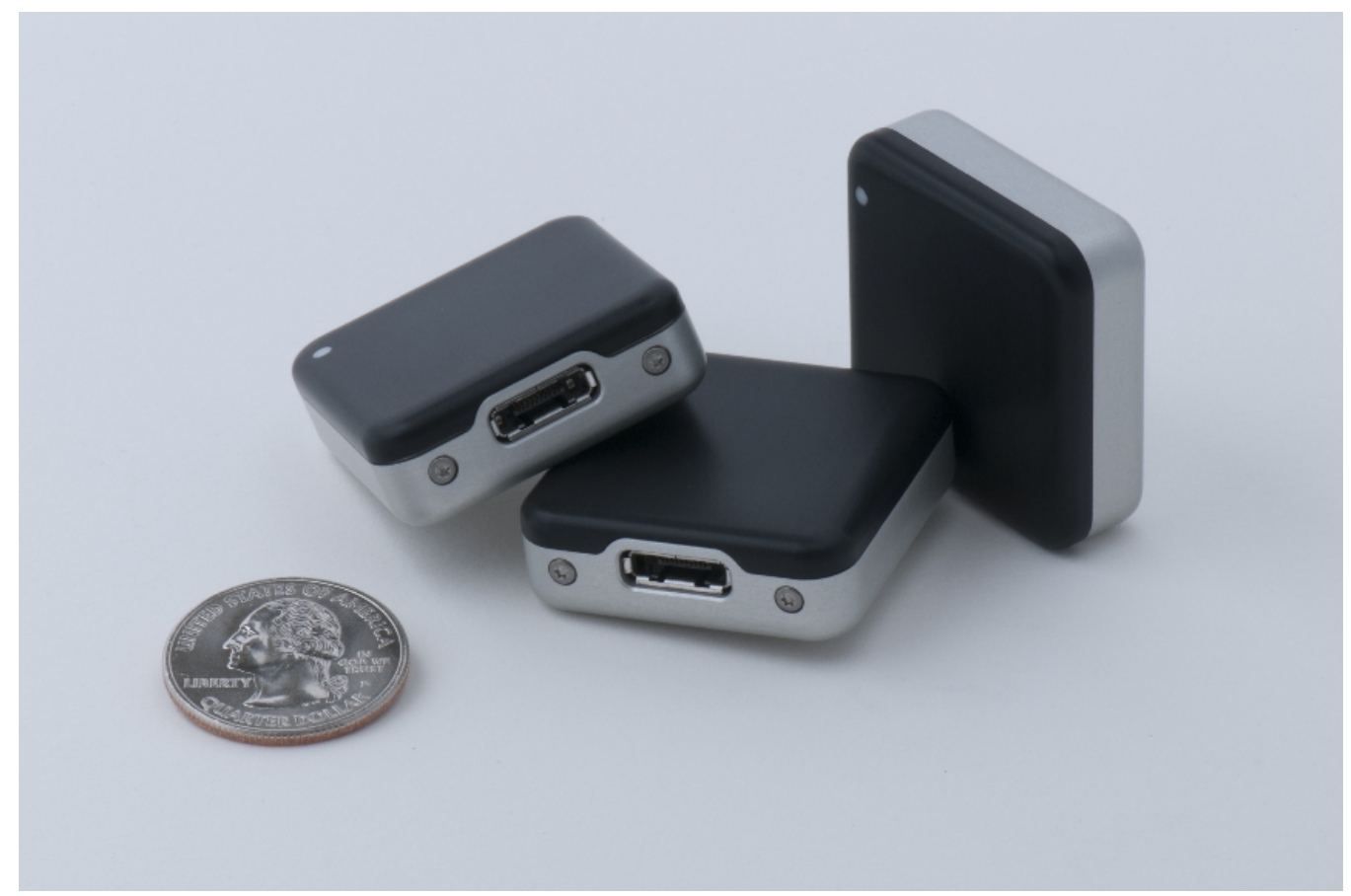

Figure 1.3: Opal Inertial measurement units used in this research (APDM Inc., Portland, Oregon, USA). Each unit contains triaxial accelerometers, gyroscopes, and magnetometers.

with exercise and treatment.

\subsection{Inertial Measurement Unit}

In this research, we collect inertial data from Opal wireless inertial measurement units (IMU) that can both log kinematic data and stream it in real-time continuously for over 8 hours (APDM Inc., Portland, Oregon). Opals use a wireless network to transmit data to a computer to analyze data in real-time. The system maintains timesynchronization of $\leq 10 \mu \mathrm{s}$ between Opals. The devices can also record data continuously and store it on an on-board memory for up to 16 hours. The device contains triaxial accelerometers, triaxial gyroscopes, and triaxial magnetometers. All channels are sampled at $128 \mathrm{~Hz}$ with 14 bits of resolution. The range of the accelerometers is $\pm 6 \mathrm{~g}$ and the range of the gyroscopes is $\pm 2000 \mathrm{deg} / \mathrm{s}$. 


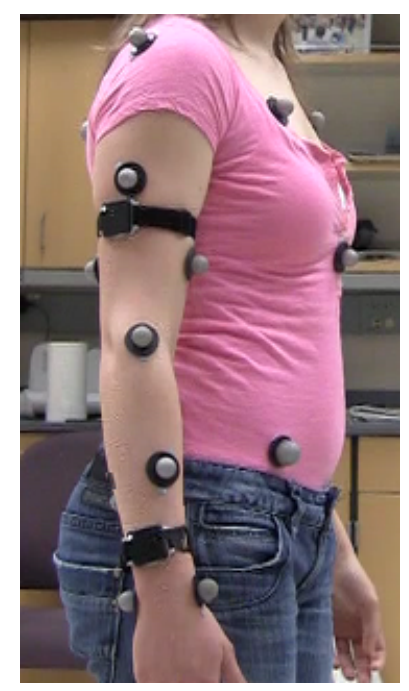

Figure 1.4: Reflective markers and Opal inertial sensors placement on the arm of one of the subjects.

Fig. 1.4 shows an example of Opal sensors attached to the upper arm and forearm during data recording. Each unit has an elastic strap to fit tightly to the arm. The figure also shows reflective markers which are part of the motion capture system used in this study as a reference system (Motion Analysis Corporation, Santa Rosa, California).

\subsection{Objectives and Significance}

To overcome many of the limitations associated with conventional motion measurement techniques, researchers are increasingly using inertial sensors for tracking and analyzing human motion. Traditionally, the orientation of a body is estimated by integrating the angular rates measured by gyroscopes attached to the moving object, starting from a known initial orientation [14]. Position is obtained by performing double integration of the translational acceleration measured by accelerometers. However, small and inexpensive inertial sensors suffer from a random bias drift that builds up over time. Integrating sensor measurements with the added growing drift 
makes it impossible to accurately determine body position and orientation for more than a few seconds. To reduce the effect of gyroscope drift on orientation estimates, some research groups use accelerometers and magnetometers in a complementary filter. In these systems, accelerometers are used to estimate inclination during static periods, and magnetometers are used to measure orientation around the vertical axis. However, this restricts accurate tracking to limited circumstances when the body is stationary and the surroundings are free of magnetic field disturbances.

The main objective of the research presented in this dissertation is to overcome the technological and methodological difficulties and limitations associated with previous human joint tracking techniques by developing an algorithm that utilizes wireless wearable inertial sensors to directly and continuously estimate human joint angles in real-time or off-line with high accuracy for slow, normal and fast complex movement with a minimal number of sensors. The algorithm combines kinematic models designed for control of robots with state space methods to accurately estimate human joint angles.

The success of the proposed technique in capturing motion and tracking dynamics of the human joints could be significant and its potential impact can be summarized as follows:

- Early diagnosis of motor function impairment: the proposed method has the potential to make it substantially easier for physicians to diagnose motor disability at an early stage of the disease.

- Therapy optimization: the proposed algorithm can be integrated in small and unobtrusive continuous movement tracking systems to provide valuable information to physicians. This continuous monitoring will help physicians to determine more accurate drug therapy dosages tailored for individuals with variable responses to therapy. 
- Physical medicine and rehabilitation: the proposed algorithm can be integrated in patients' continuous monitoring systems. This continuous monitoring will help clinicians in assessing progress of individuals undergoing physical rehabilitation in their domestic environment.

- Home biofeedback: the proposed inertial tracker can help detect subtle changes in individuals' range of motion. The tracking algorithm can be incorporated in systems to provide feedback on performance of patients working to restore damaged motor functions.

- Evaluation of new medication: the proposed method could be applied to quantitatively evaluate the efficacy of new medications.

\subsection{Dissertation Overview}

The remaining of this dissertation is organized as follows:

Chapter 2 (Literature Review) introduces the common and latest inertial tracking systems and algorithms to track human movement, and discusses their limitations. Section 2.1 reviews some of the tracking algorithms and how they are used with different sensor configuration to study human movement. Limitations of these systems and algorithms and how they are overcome in our proposed research are presented in Section 2.3.

Chapter 3 (Kinematics of Human Motion) introduces the methods used in robotics to describe rigid bodies and joint configurations, kinematic chains and the Denavit-Hartenberg convention. The recursive algorithm for propagation of angular velocity and acceleration in kinematic chains known as Newton-Euler equations is introduced in Section 3.3. The application of these methods is illustrated by introducing models of the human arm and shoulder. 
Chapter 4 (State Space) is a brief introduction to state space models and filtering. Section 4.1 introduces the problem of estimation of unobserved joint angles from noisy measurements of triaxial accelerometers and gyroscopes. Section 4.2 deals with modeling the dynamics of human joint movement and its application to the shoulder and elbow joint angles. Section 4.4 introduces validation results obtained by applying the proposed angle tracker to synthetic data generated by the model developed in Chapter 3.

In Chapter 5 (Performance Assessment), we evaluate the performance of the inertial tracking system using an optical reference tracking system. Section 5.2, introduces validation results obtained by applying the angle tracker to shoulder and elbow data collected from 8 subjects while performing various arm movements.

Chapter 6 (Modified Arm Model) introduces an improvement to the kinematic model developed in Chapter 4 to mitigate the effects of sensors drift. The new model incorporates physical constraints on the range of motion for each joint, and uses zero-velocity updates to minimize tracking errors. Performance of the tracker with the modified model is presented in Section 6.5. Joint angles calculated by the inertial tracker are compared to those obtained from a high precision industrial robot arm with six degrees of freedom, during 15-minute recordings for slow, regular and fastspeed movement of the arm.

In Chapter 7 (Particle Filter), we use a particle filter to solve the nonlinear estimation problem. Using the robot arm reference system, the performance of the particle filter-based tracker is compared to both the unscented and extended Kalman filter-based trackers to analyze the tradeoff between the improved performance and increased computational cost.

Chapter 8 (Summary And Conclusion) summarizes the study presented in this dissertation, its significance and contributions. 


\section{Literature Review}

There are a large number of publications on tracking human movement using inertial sensors for different applications. This chapter reviews some of the relevant studies with a focus on sensor configuration, tracking algorithm and their success and limitations in measuring limb kinematics. Section 2.1 reviews some of the conventional and novel inertial tracking algorithms, in the order they were published. Shortcomings and limitations of these tracking systems and algorithms are discussed in Section 2.2. Section 2.3 summarizes how these limitations are addressed in the proposed research.

\subsection{Inertial Sensors Algorithms}

Recent advances in Microelectromechanical systems (MEMS) have resulted in a significant reduction in the size and cost of inertial sensors. These advances, and the need to overcome shortcomings of other motion capture systems have led to an upsurge in research and publications on the use of wearable inertial sensors for tracking human movement.

Most studies using inertial sensors combine accelerometers and gyroscopes in wearable sensor systems. Traditionally, body orientation has been estimated by integrating the angular rate measured by gyroscopes, starting from a known initial orientation. Similarly, position is obtained by double integration of the translational acceleration measured by accelerometers. However, there is a significant problem with 
the integration of inertial sensor measurements. Inaccuracies inherent in the measurements quickly accumulate and degrade tracking accuracy. Roetenberg showed that integration of noisy gyroscope data resulted in a drift of $10-25^{\circ}$ after one minute [96]. Double integration of noisy accelerometer measurements results in a position error that grows cubically in time [46].

Willemsen et al. developed a technique to measure human joint flexion/extension angle. The system calculated the knee angle directly without integration from eight uniaxial accelerometers attached to subject's thigh and leg [120]. The lower limb was molded as a double pendulum, with the knee as a hinge joint allowing for planar rotation. The system was only accurate during slow movement [119]. To estimate knee joint angles, Williamson et al. used two inertial measurement units (IMUs) with gyroscopes and accelerometers attached to the thigh and shank. Knee flexion/extension was calculated as the difference between thigh and shank tilt angles [121]. Other researchers estimated joint angles by integrating gyroscopes and accelerometers $[114,2]$. Using a single gyroscope on the shank, Tong and Granat reported an angle error of $5^{\circ}$. Similarly, Mayagoitia et al. estimated shank and thigh inclination angles by integrating accelerometers and gyroscopes data [80]. They used eight accelerometers and two gyroscopes, and reported an error of less than $3^{\circ}$ for shank inclination angles.

To correct the drift of gyroscopes in head tracking, Foxlin et al. used occasional measurements from gravimetric tilt sensing and a complimentary Kalman filter [45]. A complimentary Kalman filter operates on the errors between orientation angle estimated by integrating gyroscope data and tilt angles from sensors. The error signals drive the Kalman filter that estimates the errors in orientation. This technique has the drawback that any head accelerations corrupts the performance of the tilt sensor, and the Kalman filter incorporates the corrupted data into the orientation estimate. To circumvent this problem in a subsequent paper [44], drift correction was performed 
only during stationary periods when it was assumed that accelerometers were sensing only gravitational acceleration. Thus, the algorithm requires that all head motion stop in order to correct inertial drift errors.

Luinge et al. showed that orientation obtained by integrating gyroscope rotational rate can be improved by fusion with inclination information obtained from accelerometers $[77,74]$. In their system, the orientation obtained by integrating angular rate was split into tilt and orientation around the vertical axis. Gravitational acceleration was used to calculate tilt during static periods. The difference between gyroscope and accelerometer tilt was used as an input to a Kalman filter to obtain a more accurate tilt angle. Estimates were then combined with rotation around the vertical axis to produce more accurate orientation angles. However, results show that estimates were accurate for only brief periods when the subject was not moving and when acceleration measurements were only due to gravity $[75,76]$.

In a gyroscope-free system, Giansanti et al. used multiple accelerometers to determine limb orientation and position [48]. They showed that, orientation estimation error was $10^{\circ}$ for a short observation interval of one second. In a second study, they combined gyroscopes with accelerometers to track position and orientation during a prescribed three task protocol: stand-to-sit, sit-to-stand and gait-initiation [47]. Estimation errors were minimal, however they restricted the application to simple tasks and limited the performance assessment to a short duration of four seconds.

Bachmann et al. used accelerometers and magnetometers in a quaternion-based complementary filter to compensate the drift in orientation obtained from angular velocity $[8,5]$. The system combined a triaxial accelerometer, a triaxial gyroscope and a triaxial magnetometer assembled to produce a sensor module referred to as Magnetic, Angular Rate and Gravity sensor (MARG). In a subsequent study, Bachmann et al. investigated the effect of electrical appliances and furniture made of ferromagnetic materials on the accuracy of orientation tracking using MARG systems [7, 6]. 
They observed errors that ranged from $12^{\circ}$ to $16^{\circ}$ and stated that these errors can be avoided by maintaining an approximate distance of two feet from the source of disturbance. This limits the success of their tracking system and restricts its use to custom laboratory environment.

Roetenberg et al. argued that errors due to magnetic field disturbance could be compensated by adequate model-based sensor fusion [97]. They developed a Kalman filter that operated on two inputs to estimate orientation of human body segments. The first is the difference between inclination from accelerometers and gyroscopes. The second input is the difference between inclination from magnetometers and gyroscopes. They included gyroscope bias error, orientation error, and magnetic disturbance as part of the process model. The filter was tested under static and dynamic conditions with ferromagnetic materials close to the sensor. Results show that orientation estimates improved significantly when the magnetic interference correction was used. However, the authors reported that the tracker was tested under controlled and limited conditions, and accuracy of orientation angle estimates could change with faster and more complex movements. In a subsequent study, Roetenberg et al. combined a body-mounted magnetic system with gyroscopes and accelerometers to track position and orientation using a complementary Kalman filter [98]. Orientation and position obtained by single and double integration of gyroscope and accelerometer data were updated with magnetic measurements to improve accuracy. The tracker was tested without metals in the vicinity, and errors were expected to grow if ferromagnetic materials were anywhere close to the magnetic system.

Sabatini et al. used a single measurement unit containing a biaxial accelerometer and one gyroscope to perform gait analysis [102]. Cyclical features of human gait were exploited to estimate stride length, walking speed, and incline from an IMU attached to the foot. Transition from one gait phase to the next was determined using the pitch angle obtained by integrating the gyroscope data. Position of the 
sensed point on the foot was obtained by double integration of the $2 \mathrm{D}$ acceleration data during the foot swing phase. Since the accelerometers could not be used to detect rotations about the vertical axis, the algorithm assumed that motion is planar, in the sagittal plane only. In a subsequent study, Sabatini developed a quaternionbased orientation tracking algorithm using triaxial gyroscopes, accelerometers and magnetometers [103]. In his extended Kalman filter (EKF) tracker, he represented rotation using quaternions rather than Euler angles to avoid trigonometric functions and singularity issues, and to improve computational efficiency.

Yun et al. used MARG sensors and a quaternion-based EKF to track human body orientation [126]. A rotary tilt table with two degrees of freedom (DOFs) was used to assess the performance of the tracker. The sensors were rotated around the $\mathrm{x}$-axis at a slow rate of $60^{\circ} / \mathrm{s}$. The authors reported a maximum error of $9^{\circ}$ for the roll angle during a dynamic period of less than $2 \mathrm{~s}$ [127]. The algorithm was also tested with human arm movement, but there was no reference system and tracking results were not reported. In a recent study, Yun et al. presented a simplified algorithm for 3 DOF orientation estimation using only accelerometers and magnetic measurements [128]. The gyroscope-free system was only suitable for tracking orientation of a static or slow moving body.

In a series of studies, Zhou et al. used two wearable inertial sensors containing gyroscopes and accelerometers to track human arm movement in a home-based rehabilitation system [129, 133]. They used Euler angles and forward kinematics to localize the wrist and elbow. Three subjects were asked to perform a set of tasks while keeping the shoulder still. Each task lasted $20 \mathrm{~s}$ and included reaching for a target, letting the arm to mimic drinking, and flexing the elbow. Results showed a high correlation between position estimates from the inertial tracker and estimates from a reference optical tracking system $[130,132]$.

Bergmann et al. developed a portable inertial system for measuring the thigh, 
knee and ankle joint angles [15]. They used six IMUs and tested their system on subjects during stair climbing. Performance of their system was compared to that of an optical reference system, and they reported an average root mean square error of $4^{\circ}$. However, their system was used to track only flexion/extension angles around one axis. In similar studies, Liu and Bagala et al. used wearable inertial sensors containing only triaxial accelerometers to estimate thigh, shank and knee angles. The study was limited to measuring only flexion/extension, and adduction/abduction during limited activities $[73,10]$.

To reduce the effect of sensor drift on orientation and position estimates, some tracking algorithms imposed limb physical constraints to confine estimates within natural range of motion. Dejnabadi et al. developed an algorithm to estimate uniaxial joint angles based on two sensor modules mounted on the shank and thigh. Each module contained two accelerometers and one gyroscope [26]. They incorporated anatomical limb constraints in the tracking system, and reported a reduction in estimation errors. The algorithm was validated by measuring knee flexion/extension angles of subjects during gait [27]. Favre et al. extended their earlier work [39], and corrected knee angle estimates based on known joint anatomical constraints using inclination data from accelerometers during static periods [38]. Luinge et al. successfully used physical constraints in the elbow to measure forearm orientation relative to the upper arm [78]. They minimized the error around the vertical axis by using the knowledge that elbow joints do not permit abduction/adduction. Despite the improvement in orientation estimates, they still reported an average error of $20^{\circ}$. Cooper et al. also used biomechanical constraints to estimate knee joint flexion/extension with 2 IMU's containing triaxial accelerometers and gyroscopes. Performance of the algorithm was evaluated with data obtained from 7 healthy subjects during walking and running. An average measurement error ranged from $0.7^{\circ}$ for slow walking to $3.4^{\circ}$ for running [22]. However, the algorithm used a simplified model of the knee 
joint to only measure a single angle.

\subsection{Limitations of Previous Studies}

As summarized in the previous section, success of inertial tracking algorithms is limited due to various factors. A typical approach in most studies discussed above involves integrating angular velocity from gyroscopes to obtain orientation, and fusing accelerometer measurements into a Kalman filter to correct the accumulation in integration drift. In these studies, orientation estimates were accurate for brief periods when acceleration was only due to gravity, and accelerometers could be used as inclinometers. Other studies utilized magnetometers to update estimates of orientation angles around the vertical axis. However, using these sensors in the vicinity of magnetic field disturbances lead to large orientation errors [20, 55, 7].

Use of the linear Kalman filter in highly nonlinear dynamics introduces more tracking errors. In some algorithms, researchers use the extended Kalman filter (EKF) to linearize the process and measurement models. The EKF is based upon linearizing the state transition and observation matrix with a first-order Taylor ex-

pansion [64]. It models the state variables with first and second order moments, which is most appropriate when the distribution is Gaussian. The linearization leads to poor performance if the dynamics are highly nonlinear [116]. The EKF also requires calculation of Jacobian matrices, which can be tedious, error prone, and time consuming.

Another factor limiting the success of inertial tracking algorithms is the number of measurement units required to track complex human movement. Large number of sensors increases the state and observation model dimensions. Consequently, increase of model dimensions and the highly nonlinear dynamic equations make the filter algorithms computationally expensive and prone to stability problems. 


\subsection{Addressing The Limitations}

Contrary to previous studies, the research presented in this dissertation demonstrates the feasibility of tracking human joint angles with minimal configuration of inertial measurement units including triaxial gyroscopes and accelerometers. As explained in Section 4.5, joint angles are directly estimated using a new tracking algorithm that does not require magnetic sensors. In addition to eliminating errors due to magnetic disturbances, this reduces the size of process and measurement models, and consequently reduces the tracker's computational cost. The algorithm utilizes both gravity and translational acceleration, together with rotational rate, making it suitable for tracking slow and fast movement with excellent accuracy. The algorithm incorporates prior knowledge of physical constraints and human natural range of motion to obtain better joint angle estimates. Another performance improvement is achieved by modeling sensor drift in the system model to mitigate the effect of random drift during long tracking periods. Furthermore, we employ zero-velocity updates to reduce the effect of gyroscope drift on estimated joint angles around the vertical axis.

The tracking system presented in this research combines the well-established kinematic models designed for control of robots with state space methods to directly estimate the joint angles from wireless wearable inertial sensors. A novel state space model is used as an integral part of the tracking system. The observation model is based on the Newton-Euler dynamics utilized in the control of robotic arms [23]. Physical constraints and human natural range of motion are incorporated in the model to enhance the tracker's performance, and to obtain accurate angle estimates during long tracking periods.

The tracker utilizes an improvement to the EKF, namely the unscented Kalman filter (UKF). The UKF removes the need for linearization while providing estimates that capture the statistics of the target distribution more accurately than the EKF [70]. The UKF approximates the distribution rather than the nonlinearity, therefore it is 
accurate to at least the second order and to the third order, if the input is Gaussian [63]. The UKF eliminates the need for Jacobian matrices and for inverse matrix calculation in EKF. A superior solution to the highly nonlinear and possibly nonGaussian estimation problem is to use sequential Monte Carlo methods, commonly known as particle filters. A particle filter $(\mathrm{PF})$ is often used as an alternative to the EKF or UKF with the advantage of better tracking if used with sufficient number of particles. However, increasing the number of particles dramatically increases the computational cost, especially with observation and process model having large dimensions. In Chapter 7, we evaluate the performance of a PF tracker, and compare it to that of the EKF and UKF in estimating joint angles. 


\section{Kinematics of Human Motion}

Kinematics is the study of body motion without consideration to the force that caused it. Kinematic analysis involves the description of movement to determine how fast a body is moving, or how far it travels. An example is the observation of leg movement, its velocity and acceleration during walking. Biomechanical models of the human body are one of the tools utilized in tracking and analyzing human movement. The goal of this chapter is to provide a description of the systematic procedures used to develop a biomechanical model of the human body. Section 3.1 provides a definition of the basic human joint angles to be estimated with our inertial tracker. Section 3.2 introduces a model for the human arm used to track shoulder and elbow joint angles. In Section 3.3, we examine kinematics of human motion by studying the propagation of velocity and acceleration in the arm. Emphasis on modeling the arm movement is only for the purpose of illustration. The technique established in this study could easily be generalized to study the motion of the head, trunk, legs or any other human motor function mechanism.

\subsection{Basic Movement Description}

There are six basic movements that occur in different joints of the human body [54]. To describe these movements, we define the relative joint angles between two segments. Description of joint movement is typically expressed relative to a starting position. This position is referred to as the fundamental position. In this position, 
the human body is upright with the head facing forward, arms at the side of the trunk with the palms facing in toward the trunk, and the legs together with the feet pointing forward.

The first pair of the six basic movements are flexion and extension. These movements occur in many joints in the body, including head, trunk, shoulder, elbow, hip and knee. Flexion is a bending movement that decreases the joint relative angle between two adjacent segments. Lifting the forearm up at the elbow is an example of elbow flexion. Extension is a straightening movement that increases the joint relative angle between two adjacent segments.

The second pair of movements are abduction and adduction. These movements are not as common as flexion and extension. They occur in the scapula, shoulder, wrist, and hip joints. Abduction is a movement away from the midline of the body. Raising an arm out to the side of the body is an example of abduction. Adduction, on the other hand, is the return movement of the segment back toward the midline of the body.

The third and last pair of basic movements are segment internal and external rotation. They occur in the head, trunk, shoulder, hip and knee joints. Internal rotation occurs when the segment rotates about the vertical axis toward the midline of the body. External rotation is the opposite movement away from the body midline.

Besides the six basic movements described above, there are a few other specialized movements at various parts of the body. While they are technically among the basic movements, they are given special names. One of these movements analyzed in this study is forearm pronation and supination. They occur as the distal end of the radius rotates over and back at the radioulnar joint. While the elbow is flexed, supination occurs in the forearm when the palm rotates to face upward. Pronation is the opposite movement to bring the palm back to face downward.

To track these complex movements, we use a common convention to develop a 


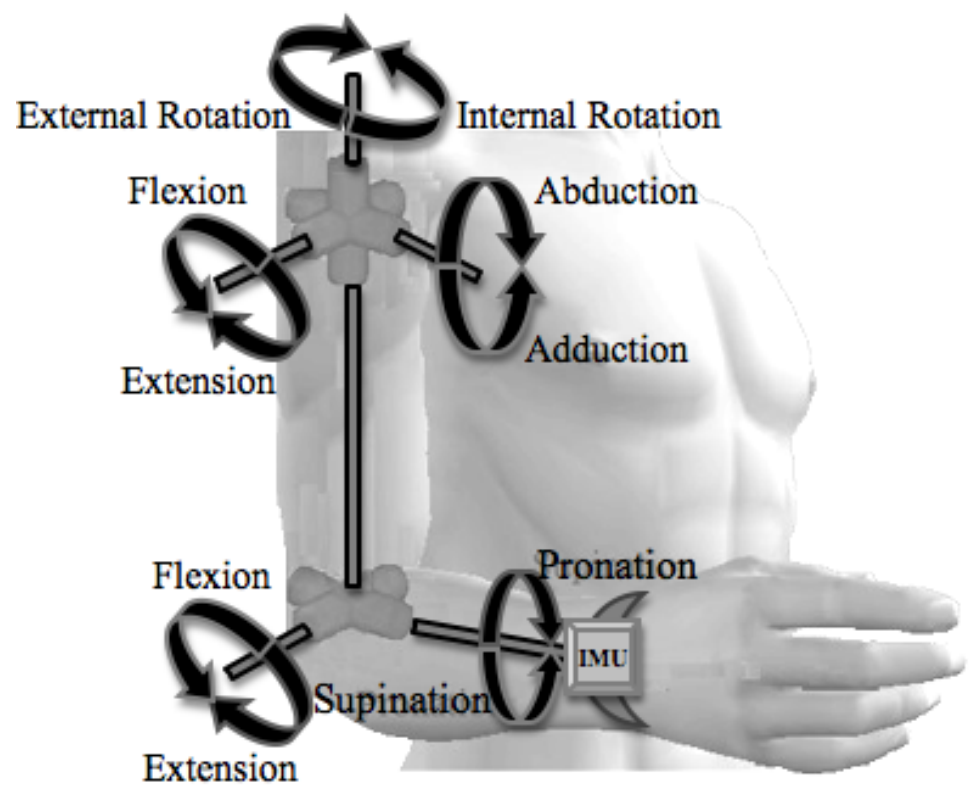

Figure 3.1: The arm is modeled with 5 degrees of freedom. Three at the shoulder to allow for flexion/extension, abduction/adduction and internal/external rotation. The fourth allows elbow flexion/extension, and the last allows forearm pronation/supination.

biomechanical model of human body segments. This is a well-established convention in robotics, and is presented in the following sections.

\subsection{Kinematic Chains and Model Development}

The human body can be represented as a system of rigid segments connected in chains by joints. The rigid segments are referred to as links, and each two adjacent links are connected by a joint. A series of rigid links is referred to as a kinematic chain. An example of a simple kinematic chain is the human arm. Shoulder, elbow and wrist joints are connected by two links: the upper arm and forearm.

The elbow joint is normally modeled as a hinge with one degree of freedom (DOF) which allows bending and straightening of the elbow (flexion and extension). Forearm supination and pronation is controlled by the radioulnar joint between the elbow and the wrist. The shoulder and the shoulder girdle make up one of the most 
complex joint groups of the human body [42]. This complex joint is typically simplified as a ball-and-socket joint with three DOFs. It allows the arm to move away from or toward the median plane of the body (abduction or adduction). Flexion and extension of the shoulder allows the arm to move up and down and is similar to that of the elbow. The shoulder also allows the arm to rotate away or toward the center of the body (external and internal rotation). These 3 DOFs are also commonly known as roll, pitch and yaw angles.

Figure 3.1 shows the arm model with five DOFs of the shoulder and elbow joints. The forearm is a link connecting the wrist and elbow joints, and the upper arm connects the elbow and shoulder joints. Craig [23] suggested that if a joint has $n$ DOFs, it can be modeled as $n$ joints of one DOF connected with $n-1$ links of zero length. Therefore, the shoulder joint could be modeled as three joints connected by two links of zero length. As shown in Figure 3.1, one inertial measurement unit (IMU) is attached to the upper arm, and a second unit is attached to the forearm above the wrist joint. Flexion of the wrist joint is not captured by the sensors and will not be considered in this model.

\subsubsection{Denavit-Hartenberg Convention}

To obtain a standard procedure for describing position and orientation angle of each link relative to its adjacent links, it is customary to attach a frame or coordinate system rigidly to each link. The position and orientation of one frame is then described with respect to its neighbor and eventually with respect to the reference frame. The convention of attaching frames to different links was proposed by Denavit and Hartenberg in 1955 and has been used since then in the analysis and control of robotic manipulators [23]. The procedure of attaching frames to links in a kinematic chain is as follows:

1. Assign a base coordinate system to some stationary reference: $X_{0}, Y_{0}, Z_{0}$, 
2. Attach a coordinate system to each of the links extending out of the reference. Frame $X_{i}, Y_{i}, Z_{i}$ is attached to link $i$ using the following conventions:

a) Align $Z_{i}$ with the axis of rotation of the $i$ th joint,

b) Establish $X_{i}$ along the common perpendicular of both $Z_{i}$ and $Z_{i+1}$, and

c) Establish $Y_{i}$ to complete the system according to the right-hand rule.

3. For the last link with frame $\{n\}$, assign $X_{n}$ freely.

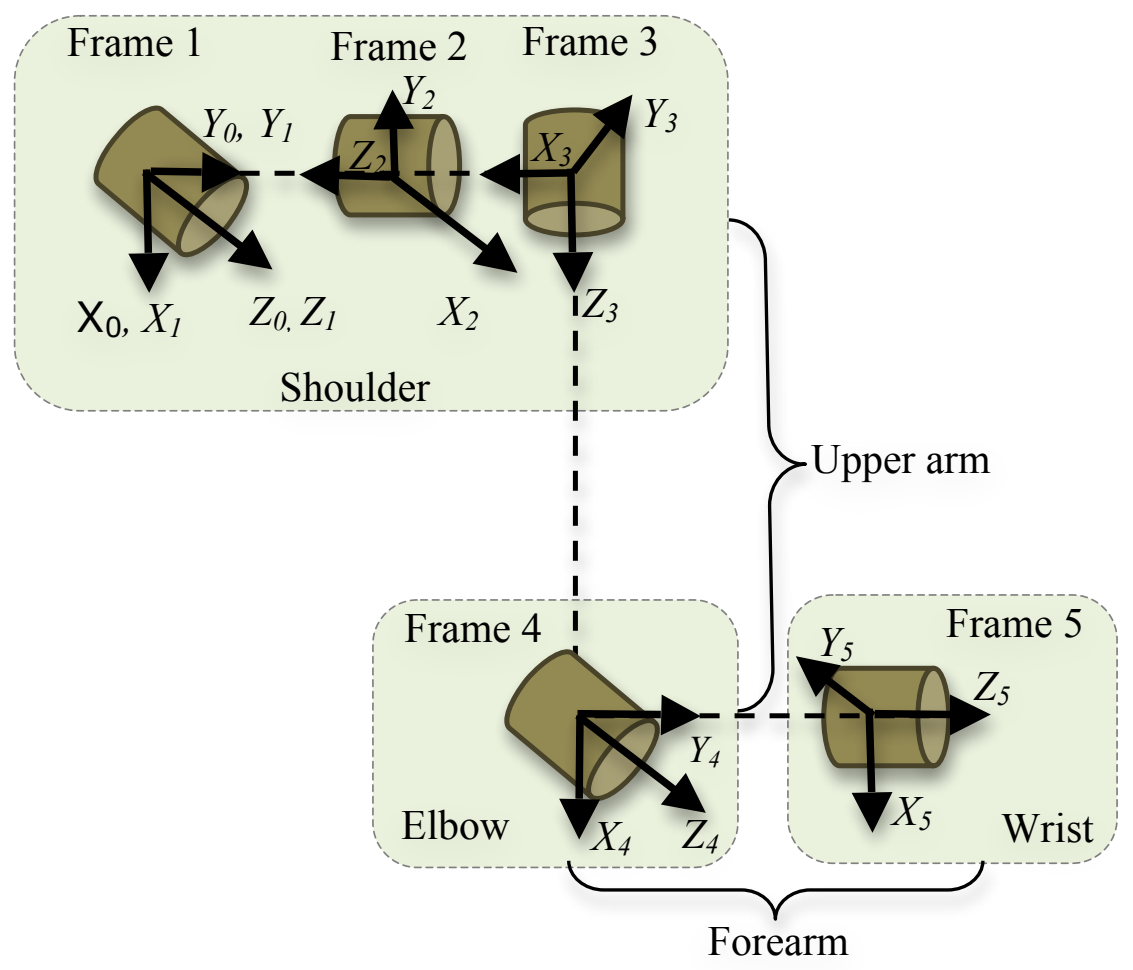

Figure 3.2: Diagram of the arm model with Frame 0 as the static reference frame. Frames 1 through 3 represent shoulder flexion/extension, abduction/adduction and internal/external rotation, respectively. Frames 4 through 5 represent the elbow flexion/extension and forearm pronation/supination.

The reference frame $\{0\}$ does not move and it simplifies calculations to align $Z_{0}$ along $Z_{1}$, and to locate frame $\{0\}$ to coincide with the initial position and orienta- 
tion of frame $\{1\}$. Similarly, for the last link with frame $\{n\}$, the direction of $X_{n}$ is chosen so that it aligns with the initial direction of $X_{n-1}$, and the origin of frame $\{n\}$ is chosen to coincide with the origin of frame $\{n-1\}$ to cause as many linkage parameters to become zero as will be seen in the following section. Figure 3.2 shows the reference frame $\{0\}$ at the center of the shoulder joint. Frames $\{1-3\}$ represent shoulder flexion/extension, abduction/adduction and internal/external rotation respectively. Frames $\{4-5\}$ represent the elbow flexion/extension and forearm pronation/supination.

To determine the relative location of each two adjacent coordinate systems, we use four parameters known as Denavit-Hartenberg Parameters.

\subsubsection{Denavit-Hartenberg Parameters}

For each pair of consecutive links, represented by their associated coordinate system, there are four parameters needed to determine the relative location of these two systems. The first parameter is the link length $a_{i}$ which is the shortest distance from $Z_{i}$ to $Z_{i+1}$ measured along the common normal $X_{i}$. This is not necessarily the anatomic length of the body segment. It is rather the biomechanical length measured along the common normal of the two axes of rotation. The second parameter is the link twist $\alpha_{i}$ which is the angle from $Z_{i}$ to $Z_{i+1}$ measured about the $X_{i}$ axis as shown in Figure 3.3. The third parameter is the distance from $X_{i-1}$ to $X_{i}$ measured along the $Z_{i}$ axis, and is known as the link offset $d_{i}$. The last and fourth parameter is the joint angle $\theta_{i}$ and it is the angle from $X_{i-1}$ to $X_{i}$ measured about the $Z_{i}$ axis as shown in Figure 3.4.

Table 3.1 shows the Denavit-Hartenberg parameters used to derive the arm links transformation. These individual transformations are used to solve for the velocity and acceleration of link $i$ relative to its neighboring link $i+1$ in the Netwon-Euler equations in section 3.3 . 


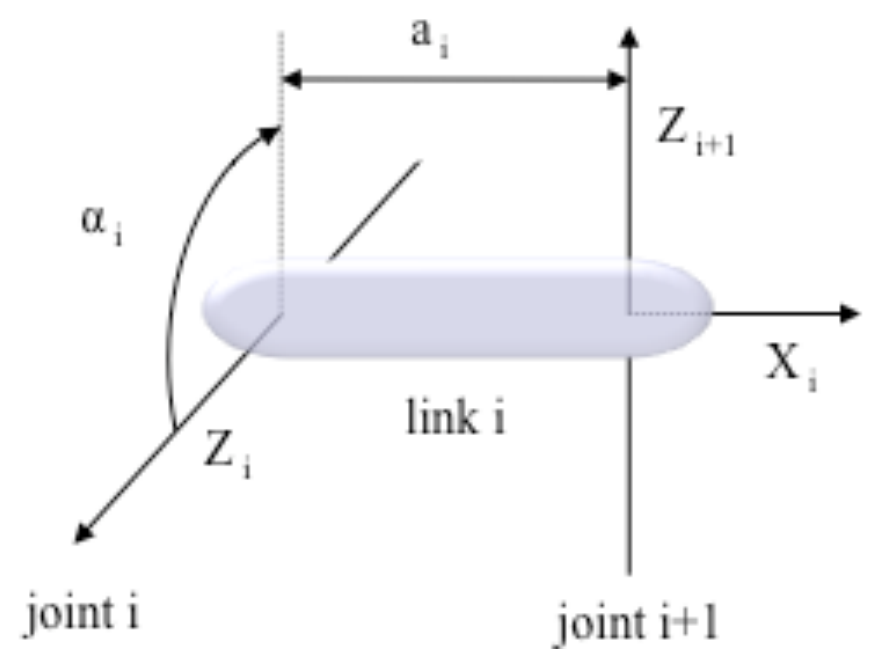

Figure 3.3: The kinematic function of a link is to maintain a fixed relationship between the two joint axes. Each link is characterized by two constants. The link length $a_{i}$ is the distance from $Z_{i}$ to $Z_{i+1}$ measured along their common normal $X_{i}$. The link twist $\alpha_{i}$ is the angle from $Z_{i}$ to $Z_{i+1}$ measured about the $X_{i}$ axis.

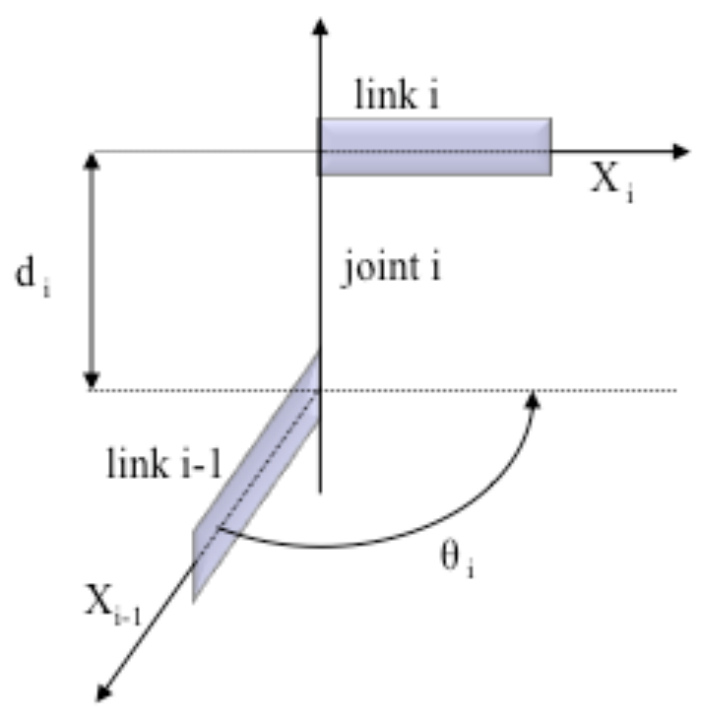

Figure 3.4: Neighboring links have a common joint axis $Z$. One parameter that defines the distance along this axis is called the link offset $d_{i}$, the distance from $X_{i-1}$ to $X_{i}$ measured along the $Z_{i}$ axis. The amount of rotation measured about the $Z_{i}$ axis is called joint angle $\theta_{i}$ and it is the angle from $X_{i-1}$ to $X_{i}$. 


\begin{tabular}{ccccc}
\hline \hline Frame & $\alpha_{i-1}$ & $a_{i-1}$ & $d_{i}$ & $\theta_{i}$ \\
\hline 1 & 0 & 0 & 0 & $\theta_{1}$ \\
2 & $\pi / 2$ & 0 & 0 & $\theta_{2}+\pi / 2$ \\
3 & $\pi / 2$ & 0 & $l_{\mathrm{u}}$ & $\theta_{3}+\pi / 2$ \\
4 & $\pi / 2$ & 0 & 0 & $\theta_{4}+\pi / 2$ \\
5 & $-\pi / 2$ & 0 & $l_{\mathrm{f}}$ & $\theta_{5}$ \\
\hline \hline
\end{tabular}

Table 3.1: Denavit-Hartenberg parameters for the shoulder and arm model. The parameter $l_{\mathrm{u}}$ is upper arm length and $l_{\mathrm{f}}$ is the forearm length. The parameters $a_{i}, \alpha_{i}$ and $d_{i}$ are constant, while $\theta_{i}$ is the joint variable that changes when link $i$ rotates with respect to link $i-1$.

\subsubsection{Link Transformations}

To relate the $i$ th frame to its adjacent frame $(i-1)$, we perform four transformations:

1. Rotate about $X_{i}$ an angle $\alpha_{i-1}$ to make the two coordinate systems coincide.

2. Translate along $X_{i}$ a distance $a_{i-1}$ to bring the two origins together.

3. Rotate about $Z_{i}$ an angle $\theta_{i}$ to align $X_{i}$ and $X_{i-1}$.

4. Translate along $Z_{i}$ a distance $d_{i}$ to bring $X_{i}$ and $X_{i-1}$ into coincidence.

Each of these four operations can be expressed by a basic rotation-translation matrix, and the product of these four transformation matrices yields a composite matrix ${ }_{i}^{i-1} T$, known as the Denavit-Hartenberg transformation matrix which defines frame $\{i\}$ to its adjacent frame $\{i-1\}$,

$$
{ }_{i}^{i-1} T=R_{x}\left(\alpha_{i-1}\right) D_{x}\left(a_{i-1}\right) R_{z}\left(\theta_{i}\right) D_{z}\left(d_{i}\right)
$$

where $R_{x}$ and $R_{z}$ are the rotation matrices about $X$ and $Z$-axis respectively, $D_{x}$ and $D_{z}$ are the translation matrices about $X$ and $Z$-axis respectively. 


$$
\begin{aligned}
{ }_{i}^{i-1} T & =\left[\begin{array}{cccc}
1 & 0 & 0 & 0 \\
0 & \cos \left(\alpha_{i-1}\right) & -\sin \left(\alpha_{i-1}\right) & 0 \\
0 & \sin \left(\alpha_{i-1}\right) & \cos \left(\alpha_{i-1}\right) & 0 \\
0 & 0 & 0 & 1
\end{array}\right]\left[\begin{array}{cccc}
1 & 0 & 0 & a_{i-1} \\
0 & 1 & 0 & 0 \\
0 & 0 & 1 & 0 \\
0 & 0 & 0 & 1
\end{array}\right] \\
& \times\left[\begin{array}{cccc}
\cos \left(\theta_{i}\right) & -\sin \left(\theta_{i}\right) & 0 & 0 \\
\sin \left(\theta_{i}\right) & \cos \left(\theta_{i}\right) & 0 & 0 \\
0 & 0 & 1 & 0 \\
0 & 0 & 0 & 1
\end{array}\right]\left[\begin{array}{cccc}
1 & 0 & 0 & 0 \\
0 & 1 & 0 & 0 \\
0 & 0 & 1 & d_{i} \\
0 & 0 & 0 & 1
\end{array}\right]
\end{aligned}
$$

multiplying out, we obtain the transformation matrix ${ }_{i}^{i-1} T$ that defines frame $\{i\}$ to its neighboring frame $\{i-1\}$ :

$$
{ }_{i}^{i-1} T=\left[\begin{array}{cccc}
\cos \left(\theta_{i}\right) & -\sin \left(\theta_{i}\right) & 0 & a_{i-1} \\
\sin \left(\theta_{i}\right) \cos \left(\alpha_{i-1}\right) & \cos \left(\theta_{i}\right) \cos \left(\alpha_{i-1}\right) & -\sin \left(\alpha_{i-1}\right) & -\sin \left(\alpha_{i-1}\right) d_{i} \\
\sin \left(\theta_{i}\right) \sin \left(\alpha_{i-1}\right) & \cos \left(\theta_{i}\right) \sin \left(\alpha_{i-1}\right) & \cos \left(\alpha_{i-1}\right) & \cos \left(\alpha_{i-1}\right) d_{i} \\
0 & 0 & 0 & 1
\end{array}\right]
$$

\subsection{Velocity and Acceleration Propagation}

To formulate the dynamic equations of the arm during movement, we use three of the Newton-Euler equations of motion. These forward recursive equations are used to propagate angular velocity, and angular and linear acceleration from the reference coordinate system through the different links of the body. Each link in motion has some angular velocity as well as angular and linear acceleration $(\omega, \dot{\omega}, \dot{v})$. 


\subsubsection{Netwon-Euler Equations}

The velocity ${ }^{i+1} \omega_{i+1}$ of link $i+1$ is that of link $i$ plus the new velocity component added by joint $i+1$.

$$
{ }^{i} \omega_{i+1}={ }^{i} \omega_{i}+{ }_{i+1}^{i} R \dot{\theta}_{i+1}{ }^{i+1} Z_{i+1}
$$

Equation (3.3) made use of the rotation matrix which is the upper left $3 \times 3$ of DenavitHartenberg matrix in equation (3.2) to relate frame $\{i\}$ to frame $\{i+1\}$ in order to represent added rotational components due to motion at the joint in frame $\{i\}$. If we multiply both sides of the equation by ${ }_{i}^{i+1} R$, we can find the description of the angular velocity of link $i+1$ with respect to frame $\{i+1\}$

$$
{ }^{i+1} \omega_{i+1}={ }_{i}^{i+1} R^{i} \omega_{i}+\dot{\theta}_{i+1}{ }^{i+1} Z_{i+1}
$$

The angular acceleration of link $i+1$ is

$$
{ }^{i+1} \dot{\omega}_{i+1}={ }_{i}^{i+1} R^{i} \dot{\omega}_{i}+{ }_{i}^{i+1} R^{i} \omega_{i} \times \dot{\theta}_{i+1}{ }^{i+1} Z_{i+1}+\ddot{\theta}_{i+1}{ }^{i+1} Z_{i+1}
$$

and the linear acceleration is given by

$$
{ }^{i+1} \dot{v}_{i+1}={ }_{i}^{i+1} R\left[{ }^{i} \dot{\omega}_{i} \times{ }^{i} P_{i+1}+{ }^{i} \omega_{i} \times\left({ }^{i} \omega_{i} \times{ }^{i} P_{i+1}\right)+{ }^{i} \dot{v}_{i}\right]
$$

where the single and double dot notation is used to represent first and second derivatives with respect to time. ${ }^{i} P_{i+1}$ is the position vector of the frame $\{i+1\}$ and it is the upper right $3 \times 1$ vector of the Denavit-Hartenberg matrix. The mathematical operation $\times$ in the above equation denotes a cross-product of two vectors and not a scalar multiplication.

Equation (3.4), (3.5) and (3.6) are fundamental to the development of the dynamic equations of the arm movement in this research. The equations are used recursively to propagate the angular velocity, and acceleration from the stationary reference system at the center of the shoulder through the links of the upper arm and forearm 
connected by the elbow joint. The dynamic equations of the arm will be presented in the following chapter.

The rotation matrices ${ }_{i}^{i+1} R$ are needed in order to utilize these forward recursive equations. They can be obtained by taking the transpose of the upper left $3 \times 3$ transformation matrix and the Denavit-Hartenberg parameters shown in Table 3.1.

$$
{ }_{i}^{i+1} R=\left[\begin{array}{ccc}
\cos \left(\theta_{i}\right) & \sin \left(\theta_{i}\right) \cos \left(\alpha_{i-1}\right) & \sin \left(\theta_{i}\right) \sin \left(\alpha_{i-1}\right) \\
-\sin \left(\theta_{i}\right) & \cos \left(\theta_{i}\right) \cos \left(\alpha_{i-1}\right) & \cos \left(\theta_{i}\right) \sin \left(\alpha_{i-1}\right) \\
0 & -\sin \left(\alpha_{i-1}\right) & \cos \left(\alpha_{i-1}\right)
\end{array}\right]
$$

We initialize the rotation velocity and acceleration as follows: $\omega_{0}=\dot{\omega}_{0}=(0,0,0)^{T}$. Linear velocity is initialized with $\dot{v}_{0}=\left(g_{x}, g_{y}, g_{z}\right)^{T}$, where $g$ is gravity.

\subsection{Summary}

In this chapter, we described joint configuration, Denavit-Hartenberg convention and parameters, and kinematic chains of the human body. This convention is used extensively in robotics, and is used in this study to model the dynamics of human movement. The arm kinematic model, with shoulder and elbow joints, was introduced based on a set of assumptions involving the known functional and anatomical features of the shoulder and elbow. A recursive algorithm for computing joint velocities and accelerations was introduced as part of the Newton-Euler equations. These equations and the arm model are utilized in the following chapter to develop the process and observation equations required in the state space framework to estimate shoulder and elbow joint angles. 


\section{Joint Angles Estimation}

In this chapter, we consider the problem of estimating unobserved states from noisy measurements. The main objective in this chapter is to model the sensor measurements as a function of joint angles. The joint angle tracking problem is expressed in a novel state space form, which incorporates a state model representing joint angles and their derivatives, and an observation model representing sensor measurements. The observation model is developed using Newton-Euler dynamics and kinematic chains described in Chapter 3. Section 4.1 introduces an overview of the estimation problem in the state space framework. A state model which describes the dynamics of human joint movements is presented in Section 4.2. Measurement equations of the inertial sensors are described in Section 4.3. To validate the state and observation models, we examine the inertial tracker performance with synthetic data generated from measurement equations of the shoulder and elbow. Validation results of the proposed tracker are presented in Section 4.4.

\subsection{Estimation and Filtering}

In estimation theory, filtering is associated with the estimation of some unobserved states of a dynamic system using a sequence of noisy measurements collected from that system [95]. The states contain relevant information required to describe the system dynamics, and their values affect the distribution of the measured data. Shoulder joint angles and their time-derivatives are an example of unobserved states, and could 
be estimated using gyroscope and accelerometer sensor measurements collected on the arm.

The primary goal is to estimate a state $x(n)$ at some time $n$, given a sequence of observations $\left.\left\{y_{n}\right\}\right|_{0} ^{N-1}$. All relevant information about the state could be estimated within a Bayesian framework [66]. The state and observations are considered as stochastic processes. The solution is found as a recursive expression for the posterior probability density function of the state, conditioned on the observed measurements. The probability density function has all statistical information required to provide a complete description of the state. Furthermore, if the the system is linear and Gaussian, only the state mean and covariance are need to completely describe the density function.

When the system is linear and noise is white, the recursive Kalman filter provides an optimal solution to the estimation problem [65]. The solution is statistically optimal with respect to any quadratic function of the estimation error [51]. In many practical estimation problems, the state or observation models are nonlinear, and use of the linear Kalman filter may result in large estimation errors. The most common approach to solving the nonlinear estimation problem is the Extended Kalman filter (EKF). It is based upon linearizing the state and observation models with a first-order Taylor expansion [81]. It models the state variables with first and second order moments, which is most appropriate when the distribution is Gaussian. If the system dynamics are highly nonlinear, this linearization provides insufficient description of the system, and consequently introduces errors due to neglecting higher order terms. The EKF also requires calculation of Jacobian matrices, which can be difficult, error prone, and time consuming.

The unscented Kalman filter (UKF) provides a better alternative to the EKF, as it removes the need for linearization while providing estimates that capture the statistics of the state distribution more accurately [63]. The UKF uses a deterministic sampling 
approach to approximate the state distribution with a minimal set of deterministically chosen sample points. These sample points completely capture the true posterior mean and covariance accurately to the $3^{\text {rd }}$ order for any nonlinearity [116].

When system models are nonlinear and non-Gaussian, the mean and covariance provide an incomplete description of the probability density function of the state. Therefore, and since their introduction, particle filters have successfully been used to provide superior solutions to the nonlinear and non-Gaussian estimation problem [50]. In particle filter (PF) algorithms, also known as Sequential Monte Carlo Methods, the state posterior distribution is approximated with a set of weighted random particles. Particle filters often provide a superior alternative to other nonlinear estimators when the system is non-Gaussian, and the state dimensions are low [19]. However, there exist systems with sufficiently high-dimensional space and complex distributions that require a large number of particles to obtain a reasonable approximation. Particle filters have computational requirements that are orders of magnitude larger than the Extended or unscented Kalman filter.

\subsection{State Space Models}

To use the Kalman filter or any of the state space-based estimators, it is necessary to express the relationship between the variables of interest and the observed data in a state space form:

$$
\begin{array}{r}
x(n+1)=f[x(n), u(n)] \\
y(n)=h[x(n), v(n)]
\end{array}
$$

where $x(n)$ represents the unobserved state of the system, and $y(n)$ is the observed or measured data. The state and measurement noise are given by $u(n)$ and $v(n)$, respectively. They are assumed to be white noise with zero mean. The functions $f[\cdot]$ and $h[\cdot]$ are the nonlinear state and observation dynamics and are assumed to 
be known. Equations (4.1) and (4.2) are also known as the process and observation equations, and they comprise the statistical state space model.

\subsubsection{State Model}

The first step in solving the estimation problem is modeling the evolution of the system. In this research, we propose a new state model to describe the dynamics of human joint movement. The states of the dynamic system are modeled as follows:

$$
\begin{aligned}
& \theta_{i}(n+1)=\theta_{i}(n)+T_{\mathrm{s}} \dot{\theta}_{i}(n)+\frac{1}{2} T_{\mathrm{s}}^{2} \ddot{\theta}_{i}(n) \\
& \dot{\theta}_{i}(n+1)=\dot{\theta}_{i}(n)+T_{\mathrm{s}} \ddot{\theta}_{i}(n) \\
& \ddot{\theta}_{i}(n+1)=\alpha \ddot{\theta}_{i}(n)+u_{\ddot{\theta}_{i}}(n)
\end{aligned}
$$

where $\theta_{i}(n)$ represents the $i^{t h}$ angle at time $n$. The rate of change in the $i^{\text {th }}$ angle is represented by angular velocity $\dot{\theta}_{i}$, and the rate of change of the angular velocity is represented by angular acceleration $\ddot{\theta}_{i}$. The sampling interval is $T_{\mathrm{s}}=f_{\mathrm{s}}^{-1}$, and $u_{\ddot{\theta}_{i}}(n)$ is a white noise process with zero mean and variance $q_{\ddot{\theta}_{i}}$. The angular acceleration is modeled as a first order autoregressive model. Autoregressive models are normally used to model many signals with an underlying autoregressive structure such as speech and seismic signals. The constant $\alpha$ has a an absolute value of $<1$ and controls how the present acceleration depends on the previous one. If $\alpha=1$, then the model becomes a random walk and the signal becomes non-stationary. If $\alpha=0$, then the angular acceleration is modeled as white noise. 


\subsubsection{Observation Model}

The observation model describes the measurement obtained by the triaxial gyroscope for the angular rate and the triaxial accelerometer for the translational acceleration

$$
y(n)=\left[\begin{array}{c}
\omega_{x}(n) \\
\omega_{y}(n) \\
\omega_{z}(n) \\
\dot{v}_{x}(n) \\
\dot{v}_{y}(n) \\
\dot{v}_{z}(n)
\end{array}\right]+\left[\begin{array}{c}
v_{g x}(n) \\
v_{g y}(n) \\
v_{g z}(n) \\
v_{a x}(n) \\
v_{a y}(n) \\
v_{a z}(n)
\end{array}\right]
$$

where $\omega_{x}, \omega_{y}$ and $\omega_{z}$ is the angular velocity along the $X, Y$ and $Z$ axes respectively. The gyroscope noise along the different axes are described by $v_{g x}, v_{g y}$ and $v_{g z}$. Similarly, the translational accelerations and their noise along the three axes are $\dot{v}_{x}, \dot{v}_{y}$ and $\dot{v}_{z}, v_{a x}, v_{a y}$ and $v_{a z}$ respectively. It should be noted that the acceleration measurement vector includes translational accelerations and the effect gravity.

\subsubsection{Nonlinear State Estimator}

The state and observation models of the shoulder and elbow joints are highly nonlinear, and the inertial tracker could be implemented with one of the nonlinear estimation algorithms described in Section 4.1. Tracking algorithms and results presented in this chapter, and in Chapters 5 and 6 utilize the unscented Kalman filter. The UKF has nearly the same computational requirements as the EKF [83], but uses a more accurate method to characterize the propagation of the state variable distribution through the nonlinear models [63]. Another advantage of the UKF is that it does not require the calculation of Jacobian matrices. In our application, the calculation of the Jacobian matrices, especially that of the measurement model, is quite involved. In Chapter 7, we evaluate the performance of the UKF tracker, and compare it to that of the EKF and PF in estimating joint angles of a 6-axis robot arm. 
Table 4.1: User-specified parameters and sample rate for the unscented Kalman filter-based joint angle tracker. $I$ represents an identity matrix.

\begin{tabular}{lcr}
\hline \hline Name & Symbol & Value \\
\hline Variance of gyroscope measurement white Gaussian noise & $\sigma_{v_{g}}{ }^{2}$ & .0001 \\
Variance of accelerometer measurement white Gaussian noise & $\sigma_{v_{a}}{ }^{2}$ & .0064 \\
Variance of process white Gaussian noise & $\sigma_{u}{ }^{2}$ & 1.00 \\
Initial state covariance matrix & $P$ & $I$ \\
Angular acceleration process parameter & $\alpha$ & 1 \\
Sample rate & $f_{s}$ & $128 \mathrm{~Hz}$ \\
Forearm length & $l_{\mathrm{f}}$ & $25 \mathrm{~cm}$ \\
Upper arm length & $l_{\mathrm{u}}$ & $25 \mathrm{~cm}$ \\
\hline \hline
\end{tabular}

The UKF has two user-specified parameters, $Q$ and $R$, which represent the process noise covariance and the measurement noise covariance. Since we assume white Gaussian noise, we set the off-diagonal entries of the two matrices to zeros. The diagonal elements of $R$ are determined empirically and account for the uncertainty in the measurement data. We approximate the measurement noise based on short static periods at both ends of sensor measurements. We used 0.001 and 0.01 for gyroscope and accelerometer noise variance. $Q$ is the process noise covariance matrix, and its diagonal elements are used as tuning parameters. These parameters control the tradeoff between certainty in the process model representing accurate motion dynamics, and how precisely the model tracks the sensor measurements. For all of results reported in this study, the angle acceleration was modeled as an autoregressive model with $\alpha=.95$. Table 4.1 lists the different parameters that were used to generate the tracking results.

\subsection{Model Validation Using Synthetic Data}

To validate the shoulder and elbow dynamic models and to examine the performance of the proposed tracker, we use synthetic data based on the state and measurement models. We use synthetic data to set a baseline for the inertial tracking algorithm 
presented in this research. Use of synthetic data allows us to control the measurement drift and bias due to sensor imperfections. Error due to these imperfections will be taken into account in the observation model presented in Chapter 6.

\subsubsection{Elbow and Forearm Model}

If we control the shoulder to keep the upper arm stationary, we obtain a model for the forearm and elbow joint. The observation equations were created with an algorithm that applies the Newton-Euler recursive equations with the parameters in Table 3.1. Equations of the inertial measurement unit placed on the forearm are:

$$
\begin{aligned}
& \omega_{x}=\dot{\theta}_{1} c \theta_{2} \\
& \omega_{y}=-\dot{\theta}_{1} s \theta_{2} \\
& \omega_{z}=\dot{\theta}_{2} \\
& \dot{v}_{x}=-l_{\mathrm{f}}\left[\dot{\theta}_{1}^{2} s \theta_{2}^{2}-\dot{\theta}_{2}^{2}\right]+g c \theta_{1} s \theta_{2} \\
& \dot{v}_{y}=-l_{\mathrm{f}}\left[\dot{\theta}_{1}^{2} s \theta_{2} c \theta_{2}+\ddot{\theta}_{2}\right]+g c \theta_{1} c \theta_{2} \\
& \dot{v}_{z}=l_{\mathrm{f}}\left[\ddot{\theta}_{1} s \theta_{2}+2 \dot{\theta}_{1} \dot{\theta}_{2} c \theta_{2}\right]-g s \theta_{1}
\end{aligned}
$$

where $\left(\omega_{x}, \omega_{y}, \omega_{z}, \dot{v}_{x}, \dot{v}_{y}, \dot{v}_{z}\right)$ are gyroscope and accelerometer data at time $n$. The time index $n$ was dropped for ease of readability. The $i^{t h}$ angle is $\theta_{i}$, the rate of change in the $i^{\text {th }}$ angle is represented by angular velocity $\dot{\theta}_{i}$, and the rate of change of the angular velocity is represented by acceleration $\ddot{\theta}_{i}$. The parameter $l_{\mathrm{f}}$ is the distance between the elbow joint center and the measurement unit placed on the forearm, near the wrist. And finally, $s \theta=\sin (\theta)$, and $c \theta=\cos (\theta)$. In this model, elbow flexion/extension is presented by $\theta_{1}$, and the forearm supination/pronation is presented by $\theta_{2}$. 


\subsubsection{Shoulder Model}

Equations of the measurement unit placed on the upper arm to estimate shoulder joint angles are:

$$
\begin{aligned}
& \omega_{x}=\dot{\theta}_{3}+\dot{\theta}_{1} s \theta_{2} \\
& \omega_{y}=\dot{\theta}_{1} c \theta_{2} s \theta_{3}-\dot{\theta}_{2} c \theta_{3} \\
& \omega_{z}=\dot{\theta}_{1} c \theta_{2} c \theta_{3}+\dot{\theta}_{2} s \theta_{3} \\
& \dot{v}_{x}=-l_{\mathrm{u}}\left[\dot{\theta}_{1}^{2} c \theta_{2}^{2}+\dot{\theta}_{2}^{2}\right]-g c \theta_{1} c \theta_{2} \\
& \dot{v}_{y}=l_{\mathrm{u}}\left[c \theta_{2} s \theta_{2} s \theta_{3} \dot{\theta}_{1}^{2}-2 \dot{\theta}_{2} c \theta_{3} s \theta_{2} \dot{\theta}_{1}+\ddot{\theta}_{2} s \theta_{3}+\ddot{\theta}_{1} c \theta_{2} c \theta_{3}\right]+g\left[c \theta_{3} s \theta_{1}+c \theta_{1} s \theta_{2} s \theta_{3}\right] \\
& \dot{v}_{z}=l_{\mathrm{u}}\left[c \theta_{2} c \theta_{3} s \theta_{2} \dot{\theta}_{1}^{2}+2 \dot{\theta}_{2} s \theta_{2} s \theta_{3} \dot{\theta}_{1}+\ddot{\theta}_{2} c \theta_{3}-\ddot{\theta}_{1} c \theta_{2} s \theta_{3}\right]-g\left[s \theta_{1} s \theta_{3}+c \theta_{1} c \theta_{3} s \theta_{2}\right]
\end{aligned}
$$

where $l_{\mathrm{u}}$ is the distance between the shoulder joint center to the measurement unit placed on the upper arm.

\subsection{Results}

We used the root mean squared error (RMSE) to quantify the difference between the actual and estimated angles. If the error between the actual and the estimated angle is defined as follows:

$$
e(n)=\theta(n)-\hat{\theta}(n)
$$

then the RMSE is

$$
\mathrm{RMSE}=\sqrt{\frac{1}{k} \sum_{n=1}^{k} e^{2}(n)}
$$

where $k$ represents the number of samples in the error.

The RMSE for both angles of elbow flexion/extension and forearm supination/pronation was $0.2^{\circ}$. Table 4.2 shows the correlation coefficient and RMSE between true and estimated angles. 
Table 4.2: Average correlation $r$, and root mean squared error RMSE between true and estimated arm angles.

\begin{tabular}{lcr}
\hline \hline Task & $r$ & $\mathrm{RMSE}\left(^{\circ}\right)$ \\
\hline Elbow Flexion/Extension & 1.0 & 0.2 \\
Forearm Supination/Pronation & 1.0 & 0.2 \\
Shoulder Flexion/Extension & 1.0 & 0.3 \\
Shoulder Abduction/Adduction & 1.0 & 0.2 \\
Shoulder Internal/External Rotation & 1.0 & 0.2 \\
\hline \hline
\end{tabular}

Elbow and forearm angle tracking results are shown in Figure 4.1. Solid blue lines represent true elbow flexion/extension (top panel) and forearm supination/pronation (bottom panel). Dotted lines represent the corresponding angles estimated by the inertial tracker. The agreement between estimated and true angles is excellent. Figure 4.2 shows the estimation error between the true and estimated elbow and forearm angles.

Figure 4.3 shows shoulder joint angle tracking results, where the solid blue line represents actual shoulder flexion/extension, adduction/abduction, and external/internal rotation angles. The dotted red line represents the corresponding angles estimated by the inertial tracker. Figure 4.4 shows the estimation error between the true and estimated shoulder angles. Maximum peak-to-peak error of $1.5^{\circ}$ occurs at peak flexion angles of $120^{\circ}$. 


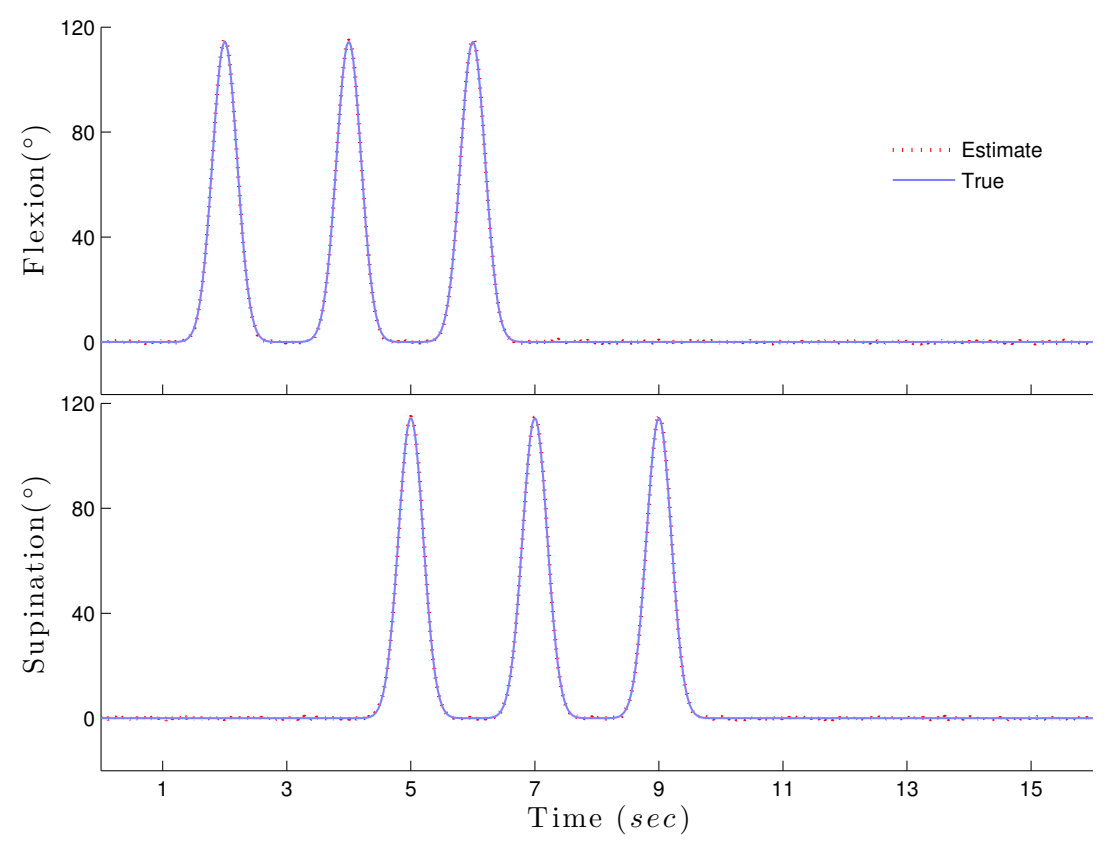

Figure 4.1: Synthetic elbow and forearm angles (solid lines), and their estimates (dotted).

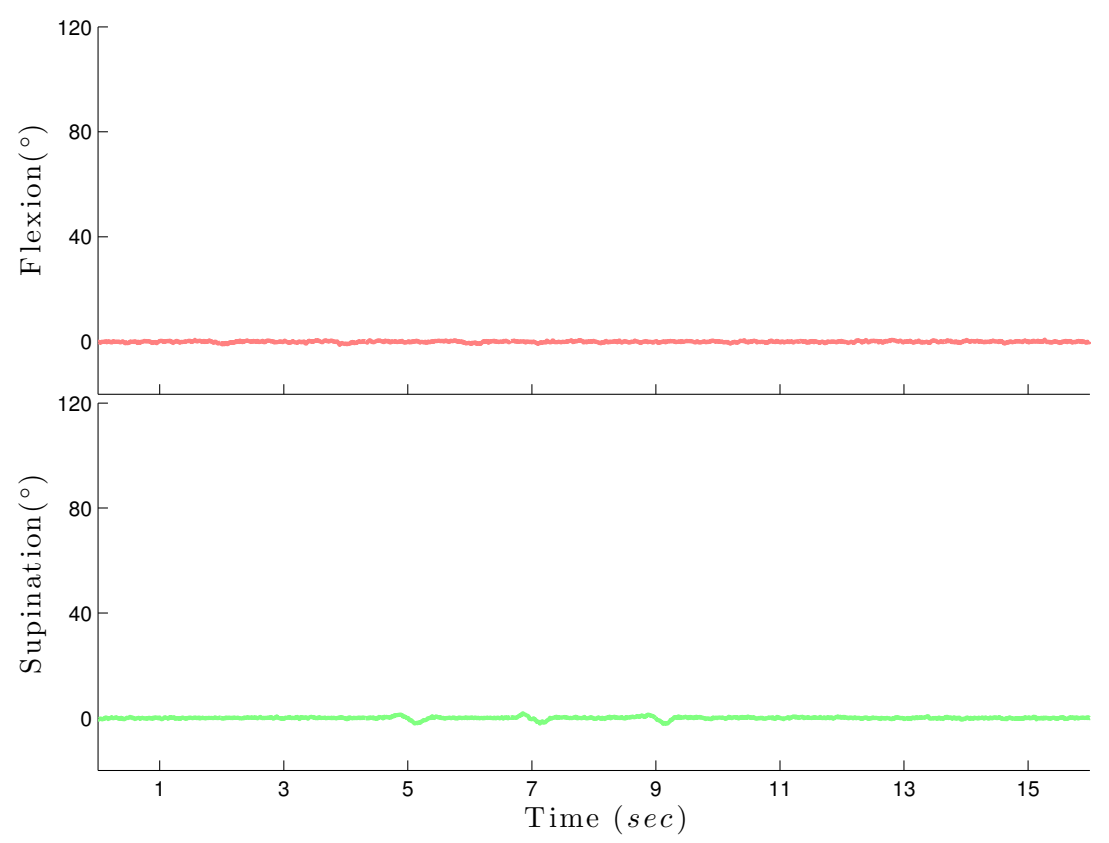

Figure 4.2: Error between true and estimated elbow flexion/extension angles (top) with $\operatorname{RMSE}=0.2^{\circ}$, and forearm supination/pronation (bottom) with $\mathrm{RMSE}=0.2^{\circ}$. 


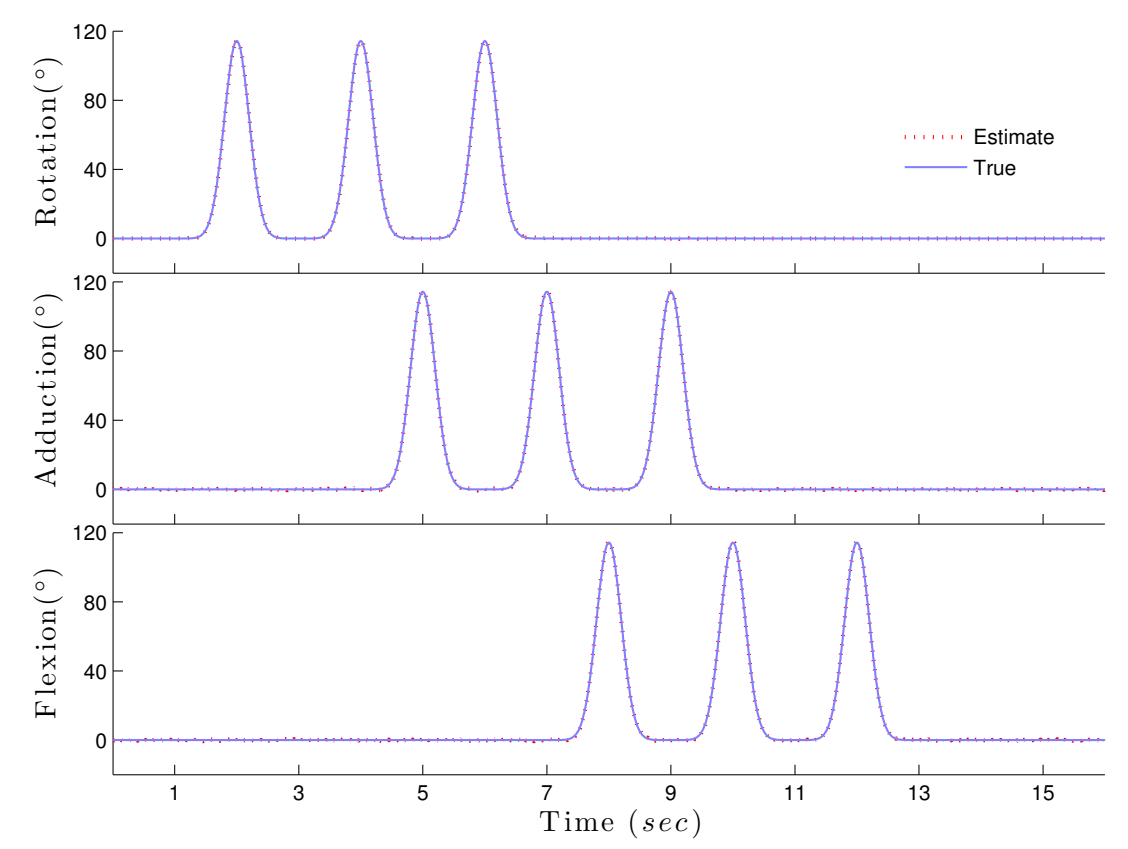

Figure 4.3: Synthetic shoulder angles (solid lines), and their estimates (dotted).

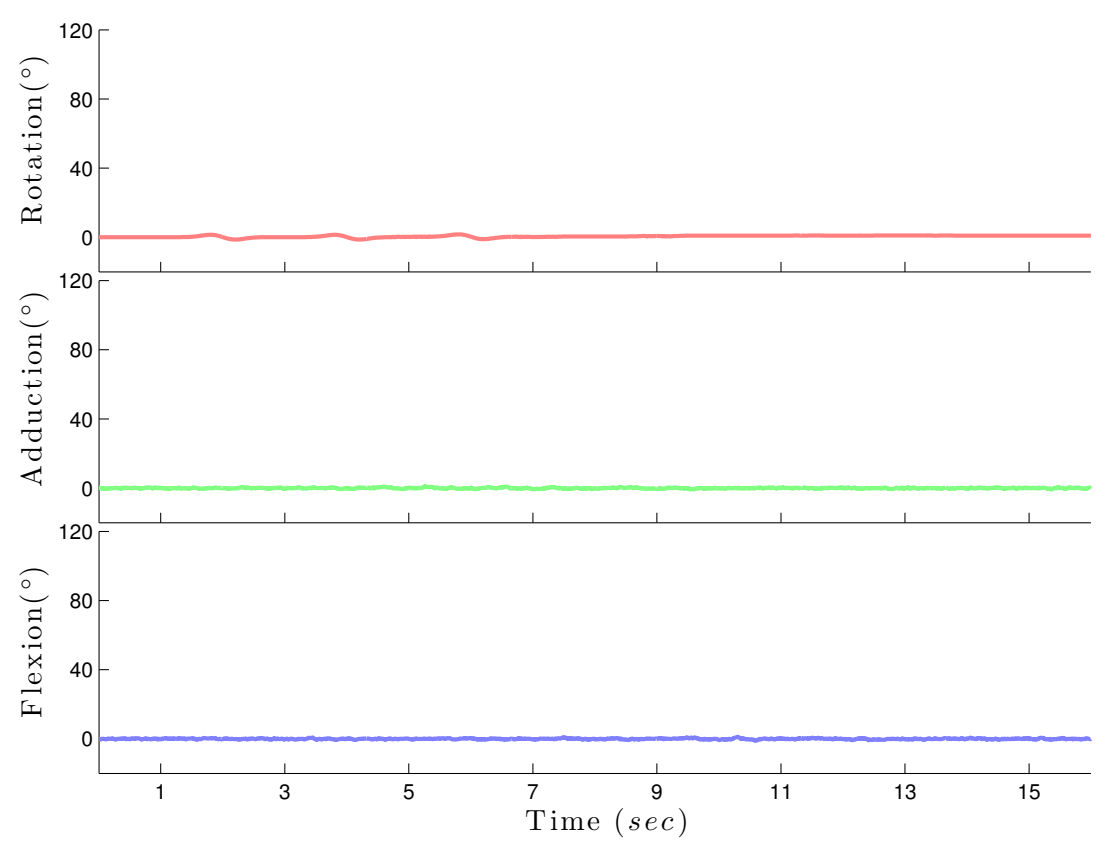

Figure 4.4: Angle estimation error of shoulder internal/external with $\mathrm{RMSE}=0.2^{\circ}$, adduction/abduction with $\mathrm{RMSE}=0.2^{\circ}$, and flexion/extension with $\mathrm{RMSE}=0.3^{\circ}$. 


\subsection{Summary}

In this chapter, we presented a brief discussion of the estimation problem in the state space form, where two models are required to analyze a dynamic system. We developed a novel state model representing joint angles and their derivatives. A novel observation model was developed using Newton-Euler dynamics and the arm kinematic chains. We used the unscented Kalman filter to implement the nonlinear state space inertial tracker. Performance of the tracker was examined using synthetic data to estimate shoulder and elbow joint angles. The algorithm and model assessment resulted in excellent tracking with very small errors and perfect correlation between true and estimated joint angles. On average, the RMS angle error was $\leq 0.3$ degrees for all shoulder and elbow angles. 


\section{Performance Assessment}

The algorithm and results introduced in this chapter were published in the IEEE Transactions on Biomedical Engineering [33]. In this chapter, we investigate the performance of our inertial tracking algorithm by comparing the estimated angles to those obtained form an optical tracking reference system. The reference system and the study protocol used to collect data from eight volunteer subjects are described in Section 5.1. A description of the full arm measurement model is presented in 5.2. We analyze normal and fast movement of the subjects performing both simple planar and more complex arm movement. Tracking results and a discussion of the performance are presented in Sections 5.3 and 5.4.

\subsection{Study Protocol}

As discussed in Chapter 1, optical systems have widely been used to assess human movements. Although they suffer from many limitations including extensive setup and calibration, restriction to indoor use and high cost, optical systems are the most common and accurate in tracking human movement [67]. Therefore, we use an optical tracker as a reference system to evaluate the performance of our inertial tracking algorithm.

Success and limitations of previous tracking algorithms using inertial sensors were discussed in Chapter 2. Most of these algorithms were only applicable under

limited circumstances. Some studies restricted the application to simple planar tasks. 
In other studies, the estimation was accurate for only brief periods. Others reported large orientation errors due to limitations of the inertial devices used to capture the motion.

To compare the performance of the inertial and optical tracking systems, we collected two data sets from a total of 8 subjects performing tasks described in Table 5.1. The study was conducted in the Balance Disorders Laboratory at Oregon Health \& Science University (OHSU). The laboratory is equipped with a motion capture system with eight high-speed infrared cameras (Motion Analysis Corporation, Santa Rosa, California, USA). The cameras recorded the position of 14 reflective markers placed on the sternum, upper arm, forearm, shoulder, elbow and wrist, as shown in Fig. 5.1.

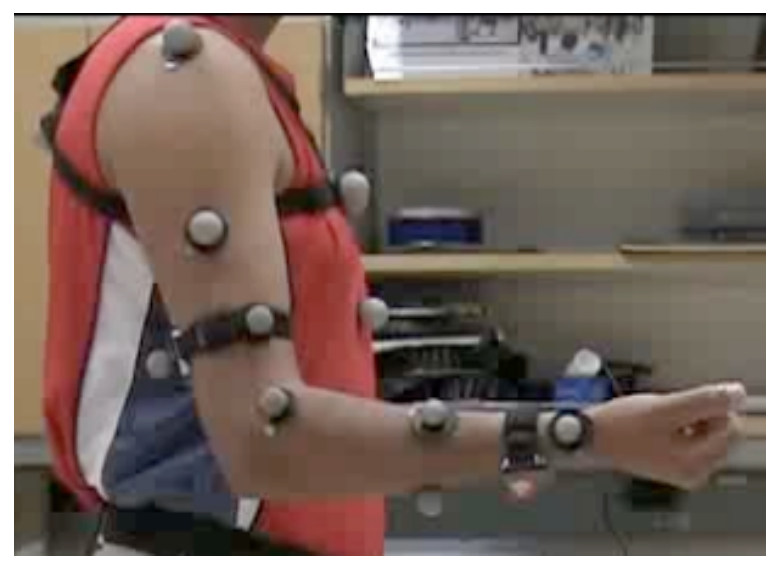

Figure 5.1: Reflective markers and Opal inertial measurement units placement on the upper arm and forearm of one of the subjects.

Elbow and forearm angles were obtained from 3D positions of the markers placed on the upper arm and forearm, based on the algorithm described in [52]. Similarly, shoulder angles were obtained from positions of the reflective markers placed on the shoulder and upper arm. One Opal inertial measurement unit (IMU), containing triaxial accelerometers and gyroscopes, was placed on the upper arm approximately halfway between the shoulder and elbow joints. Another Opal unit was placed on the 
forearm, near the wrist. Opal is a miniature wireless inertial measurement unit that streams kinematic data in real-time (APDM, Inc., Portland, OR). The units maintain time-synchronization of $\leq 10 \mu \mathrm{s}$. Each IMU was attached to the arm with a strap band centered within a cluster of 4 markers. A stationary calibration period of $3 \mathrm{~s}$ at the initial pose preceded each movement task. The calibration period served multiple purposes. The first was to align the inertial and optical reference systems. The second was to calculate the variance of sensor measurement noise. The stationary period was also used to calculate the gyroscope constant bias. This bias was removed from the gyroscope data before calculating the joint angles.

Inertial and optical reference systems were synchronized to start and stop recording simultaneously using an access point. Inertial data was originally sampled at $128 \mathrm{~Hz}$, and the Vicon optical data at $60 \mathrm{~Hz}$. Angles calculated from inertial sensors were then re-sampled to $60 \mathrm{~Hz}$ for comparison with angles obtained from the optical system.

\subsubsection{Data Collection}

Subjects were asked to perform a set of arm movements involving only rotation of the shoulder and elbow joints. During the prescribed tasks, subjects were instructed to keep the rest of the body stationary, especially the trunk. We collected two different data measurements from eight subjects. For the first data set, four subjects were asked to repeat simple planar articulations. Planar movement included only one of the five angles at a time. Each recording lasted $18 \mathrm{~s}$, including a stationary calibration period of $3 \mathrm{~s}$ at the initial pose as shown in Fig. 5.1. Subjects were informed of when to start and stop by the sound of a metronome which was also used to guide subjects' movement rate. Recording was stopped between each task while subjects returned to the initial pose. Articulations were performed at a comfortable rate similar to that of daily activities with an average rotation rate of $180^{\circ} / \mathrm{s}$. To verify the performance of 
the algorithm in tracking fast movement, we asked the subjects to perform the same tasks at a faster pace. This articulation was performed at an average rate of $420^{\circ} / \mathrm{s}$.

In the second data set, to obtain a longer measurement recording, the other four subjects performed the same tasks described in Table 5.1 continuously without stopping the recording between tasks. Subjects started with the simple planar articulations, and ended with more complex movement in order to involve both the shoulder and elbow angles simultaneously. These movements included having the subjects start with their arm at their side, reaching to touch their nose with their index finger, bringing their arm back to the starting position, and repeating five times. Subjects were also asked to mimic reaching for an imaginary doorknob and turning it, repeating this entire motion five times as well. On average, the continuous recording lasted 2 minutes. Each recording was repeated 3 times by each subject with two brief rest periods.

\subsection{Shoulder and Elbow Measurement Model}

The discrete time state space model was introduced in Chapter 4. Our state model equations are given by:

$$
\begin{aligned}
& \theta_{i}(n+1)=\theta_{i}(n)+T_{\mathrm{s}} \dot{\theta}_{i}(n)+\frac{1}{2} T_{\mathrm{s}}^{2} \ddot{\theta}_{i}(n) \\
& \dot{\theta}_{i}(n+1)=\dot{\theta}_{i}(n)+T_{\mathrm{s}} \ddot{\theta}_{i}(n) \\
& \ddot{\theta}_{i}(n+1)=\alpha \ddot{\theta}_{i}(n)+u_{\ddot{\theta}_{i}}(n)
\end{aligned}
$$

where $i=\{1, \ldots, 5\}$ of the five angles, $\theta_{i}(n)$ is the $i^{\text {th }}$ angle at time $n$. Angles $\theta_{1}$ through $\theta_{3}$ represent shoulder flexion/extension, abduction/adduction, and internal/external rotation, respectively. The angle $\theta_{4}$ represents elbow flexion/extension, and $\theta_{5}$ represents forearm supination/pronation. Similarly, $\dot{\theta}_{i}$ and $\ddot{\theta}_{i}$ are the time derivatives of the $i^{\text {th }}$ angle $\theta$, and they represent angular velocity and angular acceleration. 
Observation equations of the IMU placed on the upper arm were introduced in Section 4.3.2. Equations of the forearm measurement unit depend on the 5 joint angles, and are presented below. These large measurement equations were generated with an algorithm that algebraically applies the arm kinematic model parameters to Newton-Euler equations. We developed a recursive routine using symbolic math manipulation in the Matlab programming environment (The MathWorks, Inc., Natick, MA, USA) to generate the following measurement equations: 


$$
\begin{aligned}
& \omega_{x}=c \theta_{5}\left(c \theta_{4}\left(\dot{\theta}_{2} c \theta_{3}+\dot{\theta}_{1} c \theta_{3} c \theta_{2}\right)+c \theta_{4}\left(\dot{\theta}_{3}-\dot{\theta}_{1} c \theta_{2}\right)\right)-s \theta_{5}\left(\dot{\theta}_{4}-\dot{\theta}_{2} c \theta_{3}+\dot{\theta}_{1} c \theta_{2} c \theta_{3}\right) \\
& \omega_{y}=-c \theta_{5}\left(\dot{\theta}_{4}-\dot{\theta}_{2} c \theta_{3}+\dot{\theta}_{1} c \theta_{2} c \theta_{3}\right)-s \theta_{5}\left(c \theta_{4}\left(\dot{\theta}_{2} c \theta_{3}+\dot{\theta}_{1} c \theta_{3} c \theta_{2}\right)+c \theta_{4}\left(\dot{\theta}_{3}-\dot{\theta}_{1} c \theta_{2}\right)\right) \\
& \omega_{z}=\dot{\theta}_{5}-c \theta_{4}\left(\dot{\theta}_{2} c \theta_{3}+\dot{\theta}_{1} c \theta_{3} c \theta_{2}\right)+c \theta_{4}\left(\dot{\theta}_{3}-\dot{\theta}_{1} c \theta_{2}\right) \\
& \dot{v}_{x}=-c \theta_{5}\left(c \theta _ { 4 } \left(c \theta_{3}\left(l_{\mathrm{u}}\left(\ddot{\theta}_{1} c \theta_{2}+\dot{\theta}_{1} \dot{\theta}_{2} c \theta_{2}\right)+g s \theta_{1}+l_{\mathrm{u}} \dot{\theta}_{1} \dot{\theta}_{2} c \theta_{2}\right)+c \theta_{3}\left(l_{\mathrm{u}} c \theta_{2} c \theta_{2} \dot{\theta}_{1}^{2}-l_{\mathrm{u}} \ddot{\theta}_{2}\right.\right.\right. \\
& \left.\left.+g c \theta_{1} c \theta_{2}\right)\right)+c \theta_{4}\left(l_{\mathrm{u}} \dot{\theta}_{1}^{2} c \theta_{2}^{2}+l_{\mathrm{u}} \dot{\theta}_{2}^{2}+g c \theta_{1} c \theta_{2}\right)+l_{\mathrm{f}}\left(\ddot{\theta}_{4}-\ddot{\theta}_{2} c \theta_{3}+c \theta_{3}\left(\ddot{\theta}_{1} c \theta_{2}+\dot{\theta}_{1} \dot{\theta}_{2} c \theta_{2}\right)\right. \\
& \left.+\dot{\theta}_{3}\left(\dot{\theta}_{2} c \theta_{3}+\dot{\theta}_{1} c \theta_{3} c \theta_{2}\right)\right)+l_{\mathrm{f}}\left(c \theta_{4}\left(\dot{\theta}_{2} c \theta_{3}+\dot{\theta}_{1} c \theta_{3} c \theta_{2}\right)+c \theta_{4}\left(\dot{\theta}_{3}-\dot{\theta}_{1} c \theta_{2}\right)\right)\left(c \theta _ { 4 } \left(\dot{\theta}_{2} c \theta_{3}\right.\right. \\
& \left.\left.\left.+\dot{\theta}_{1} c \theta_{3} c \theta_{2}\right)-c \theta_{4}\left(\dot{\theta}_{3}-\dot{\theta}_{1} c \theta_{2}\right)\right)\right)-s \theta_{5}\left(l _ { \mathrm { f } } \left(c \theta_{4}\left(\ddot{\theta}_{3}-\ddot{\theta}_{1} c \theta_{2}+\dot{\theta}_{1} \dot{\theta}_{2} c \theta_{2}\right)-\dot{\theta}_{4}\left(c \theta _ { 4 } \left(\dot{\theta}_{2} c \theta_{3}\right.\right.\right.\right. \\
& \left.\left.+\dot{\theta}_{1} c \theta_{3} c \theta_{2}\right)-c \theta_{4}\left(\dot{\theta}_{3}-\dot{\theta}_{1} c \theta_{2}\right)\right)+c \theta_{4}\left(c \theta_{3}\left(\ddot{\theta}_{1} c \theta_{2}+\dot{\theta}_{1} \dot{\theta}_{2} c \theta_{2}\right)+\dot{\theta}_{3}\left(\dot{\theta}_{2} c \theta_{3}-\dot{\theta}_{1} c \theta_{2} c \theta_{3}\right)\right. \\
& \left.\left.+\ddot{\theta}_{2} c \theta_{3}\right)\right)-c \theta_{3}\left(l_{\mathrm{u}} c \theta_{2} c \theta_{2} \dot{\theta}_{1}^{2}-l_{\mathrm{u}} \ddot{\theta}_{2}+g c \theta_{1} c \theta_{2}\right)+c \theta_{3}\left(l_{\mathrm{u}}\left(\ddot{\theta}_{1} c \theta_{2}+\dot{\theta}_{1} \dot{\theta}_{2} c \theta_{2}\right)+g s \theta_{1}\right. \\
& \left.\left.+l_{\mathrm{u}} \dot{\theta}_{1} \dot{\theta}_{2} c \theta_{2}\right)-l_{\mathrm{f}}\left(c \theta_{4}\left(\dot{\theta}_{2} c \theta_{3}+\dot{\theta}_{1} c \theta_{3} c \theta_{2}\right)-c \theta_{4}\left(\dot{\theta}_{3}-\dot{\theta}_{1} c \theta_{2}\right)\right)\left(\dot{\theta}_{4}-\dot{\theta}_{2} c \theta_{3}+\dot{\theta}_{1} c \theta_{2} c \theta_{3}\right)\right) \\
& \dot{v}_{y}=s \theta_{5}\left(c \theta _ { 4 } \left(c \theta_{3}\left(l_{\mathrm{u}}\left(\ddot{\theta}_{1} c \theta_{2}+\dot{\theta}_{1} \dot{\theta}_{2} c \theta_{2}\right)+g s \theta_{1}+l_{\mathrm{u}} \dot{\theta}_{1} \dot{\theta}_{2} c \theta_{2}\right)+c \theta_{3}\left(l_{\mathrm{u}} c \theta_{2} c \theta_{2} \dot{\theta}_{1}^{2}-l_{\mathrm{u}} \ddot{\theta}_{2}\right.\right.\right. \\
& \left.\left.+g c \theta_{1} c \theta_{2}\right)\right)+c \theta_{4}\left(l_{\mathrm{u}} \dot{\theta}_{1}^{2} c \theta_{2}^{2}+l_{\mathrm{u}} \dot{\theta}_{2}^{2}+g c \theta_{1} c \theta_{2}\right)+l_{\mathrm{f}}\left(\ddot{\theta}_{4}-\ddot{\theta}_{2} c \theta_{3}+c \theta_{3}\left(\ddot{\theta}_{1} c \theta_{2}+\dot{\theta}_{1} \dot{\theta}_{2} c \theta_{2}\right)\right. \\
& \left.+\dot{\theta}_{3}\left(\dot{\theta}_{2} c \theta_{3}+\dot{\theta}_{1} c \theta_{3} c \theta_{2}\right)\right)+l_{\mathrm{f}}\left(c \theta_{4}\left(\dot{\theta}_{2} c \theta_{3}+\dot{\theta}_{1} c \theta_{3} c \theta_{2}\right)+c \theta_{4}\left(\dot{\theta}_{3}-\dot{\theta}_{1} c \theta_{2}\right)\right)\left(c \theta _ { 4 } \left(\dot{\theta}_{2} c \theta_{3}\right.\right. \\
& \left.\left.\left.+\dot{\theta}_{1} c \theta_{3} c \theta_{2}\right)-c \theta_{4}\left(\dot{\theta}_{3}-\dot{\theta}_{1} c \theta_{2}\right)\right)\right)-c \theta_{5}\left(l _ { \mathrm { f } } \left(c \theta_{4}\left(\ddot{\theta}_{3}-\ddot{\theta}_{1} c \theta_{2}+\dot{\theta}_{1} \dot{\theta}_{2} c \theta_{2}\right)-\dot{\theta}_{4}\left(c \theta _ { 4 } \left(\dot{\theta}_{2} c \theta_{3}\right.\right.\right.\right. \\
& \left.\left.+\dot{\theta}_{1} c \theta_{3} c \theta_{2}\right)-c \theta_{4}\left(\dot{\theta}_{3}-\dot{\theta}_{1} c \theta_{2}\right)\right)+c \theta_{4}\left(c \theta_{3}\left(\ddot{\theta}_{1} c \theta_{2}+\dot{\theta}_{1} \dot{\theta}_{2} c \theta_{2}\right)+\dot{\theta}_{3}\left(\dot{\theta}_{2} c \theta_{3}-\dot{\theta}_{1} c \theta_{2} c \theta_{3}\right)\right. \\
& \left.\left.+\ddot{\theta}_{2} c \theta_{3}\right)\right)-c \theta_{3}\left(l_{\mathrm{u}} c \theta_{2} c \theta_{2} \dot{\theta}_{1}^{2}-l_{\mathrm{u}} \ddot{\theta}_{2}+g c \theta_{1} c \theta_{2}\right)+c \theta_{3}\left(l_{\mathrm{u}}\left(\ddot{\theta}_{1} c \theta_{2}+\dot{\theta}_{1} \dot{\theta}_{2} c \theta_{2}\right)+g s \theta_{1}\right. \\
& \left.\left.+l_{\mathrm{u}} \dot{\theta}_{1} \dot{\theta}_{2} c \theta_{2}\right)-l_{\mathrm{f}}\left(c \theta_{4}\left(\dot{\theta}_{2} c \theta_{3}+\dot{\theta}_{1} c \theta_{3} c \theta_{2}\right)-c \theta_{4}\left(\dot{\theta}_{3}-\dot{\theta}_{1} c \theta_{2}\right)\right)\left(\dot{\theta}_{4}-\dot{\theta}_{2} c \theta_{3}+\dot{\theta}_{1} c \theta_{2} c \theta_{3}\right)\right) \\
& \dot{v}_{z}=c \theta_{4}\left(c \theta_{3}\left(l_{\mathrm{u}}\left(\ddot{\theta}_{1} c \theta_{2}+\dot{\theta}_{1} \dot{\theta}_{2} c \theta_{2}\right)+g s \theta_{1}+l_{\mathrm{u}} \dot{\theta}_{1} \dot{\theta}_{2} c \theta_{2}\right)+c \theta_{3}\left(l_{\mathrm{u}} c \theta_{2} c \theta_{2} \dot{\theta}_{1}^{2}-l_{\mathrm{u}} \ddot{\theta}_{2}\right.\right. \\
& \left.\left.+g c \theta_{1} c \theta_{2}\right)\right)-l_{\mathrm{f}}\left(\dot{\theta}_{4}-\dot{\theta}_{2} c \theta_{3}+\dot{\theta}_{1} c \theta_{2} c \theta_{3}\right)^{2}-l_{\mathrm{f}}\left(c \theta_{4}\left(\dot{\theta}_{2} c \theta_{3}+\dot{\theta}_{1} c \theta_{3} c \theta_{2}\right)\right. \\
& \left.+c \theta_{4}\left(\dot{\theta}_{3}-\dot{\theta}_{1} c \theta_{2}\right)\right)^{2}-c \theta_{4}\left(l_{\mathrm{u}} \dot{\theta}_{1}^{2} c \theta_{2}^{2}+l_{\mathrm{u}} \dot{\theta}_{2}^{2}+g c \theta_{1} c \theta_{2}\right)
\end{aligned}
$$

where $l_{\mathrm{f}}$ is the distance between the elbow joint and the IMU placed on the forearm. Similarly, $l_{\mathrm{u}}$ is the distance between the shoulder joint and the IMU placed on the upper arm. 


\subsection{Results}

To quantify the tracking performance and compare angle estimates from both systems, we calculated the correlation coefficient $r$, and the RMSE between angle estimates from the inertial and optical tracking systems. On average, the correlation coefficient was $r \geq 0.97$ for all tasks among all subjects. Table 5.1 shows the average correlation coefficients, average RMSE across subjects for all tasks, and the average peak-to-peak error between inertial and optical angles.

Table 5.1: Average correlation $r$, RMSE, and peak-to-peak error between optical and inertial angles of shoulder and elbow joints.

\begin{tabular}{lccc}
\hline \hline Task & $r$ & $\operatorname{RMSE}\left({ }^{\circ}\right)$ & Peak Error $\left(^{\circ}\right)$ \\
\hline Elbow Flexion/Extension & 0.98 & 6.5 & 9.8 \\
Forearm Supination/Pronation & 0.95 & 5.5 & 7.8 \\
Shoulder Flexion/Extension & 0.98 & 5.5 & 7.9 \\
Shoulder Abduction/Adduction & 0.99 & 4.4 & 8.1 \\
\hline \hline
\end{tabular}

Fig. 5.2 shows elbow and forearm angles for one of the subjects performing simple planar rotation at normal daily life movement rate. The top panel of the figure shows elbow flexion/extension estimates using the inertial tracker (solid blue line) and the optical system (dashed red line). Similarly, the bottom panel of the figure shows forearm supination/pronation estimates from both tracking systems. The agreement between angle estimates from both systems is excellent, consequently the red line representing optical angles is concealed by the blue line representing inertial angles in Fig. 5.2. The difference between elbow and forearm angle estimates from both tracking systems is presented in Fig. 5.3. The figure shows small errors for both angles, with slightly larger errors for estimates of the forearm supination/pronation angles. 


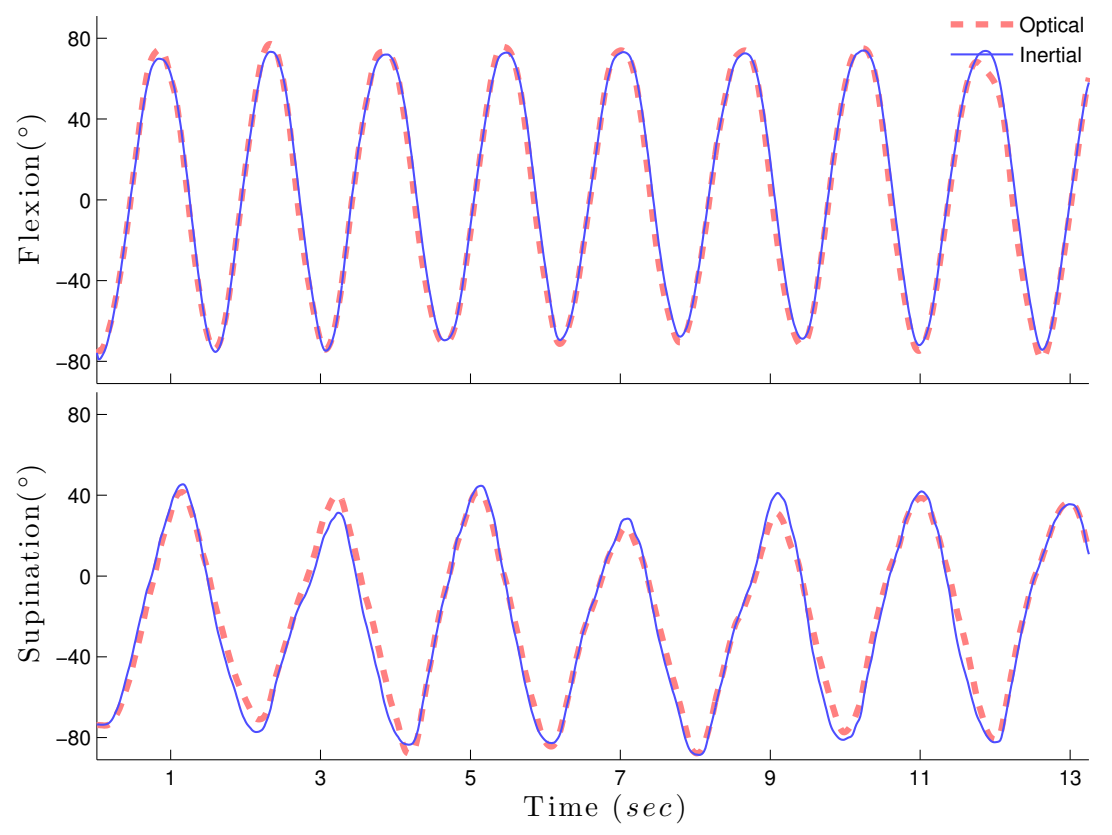

Figure 5.2: Elbow and forearm angle estimates by the optical system (dashed red line) compared to inertial angles estimate (solid blue line).

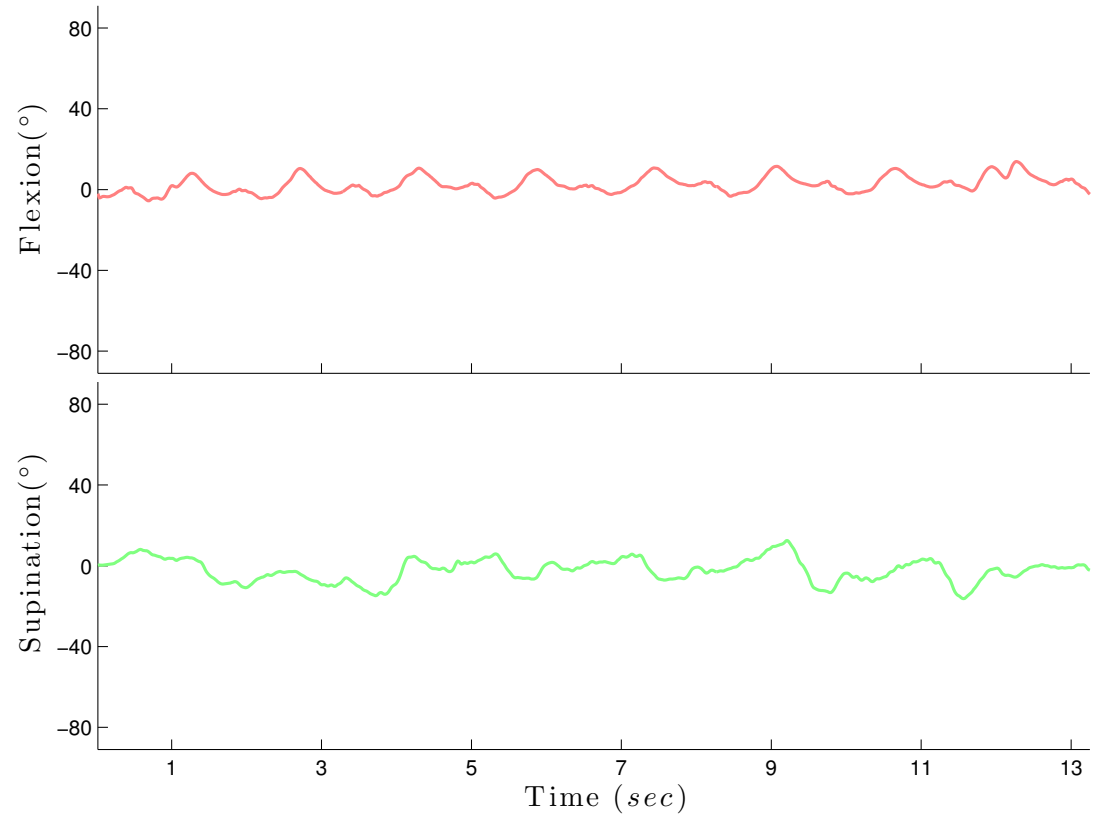

Figure 5.3: Difference between estimates using optical and inertial trackers. 


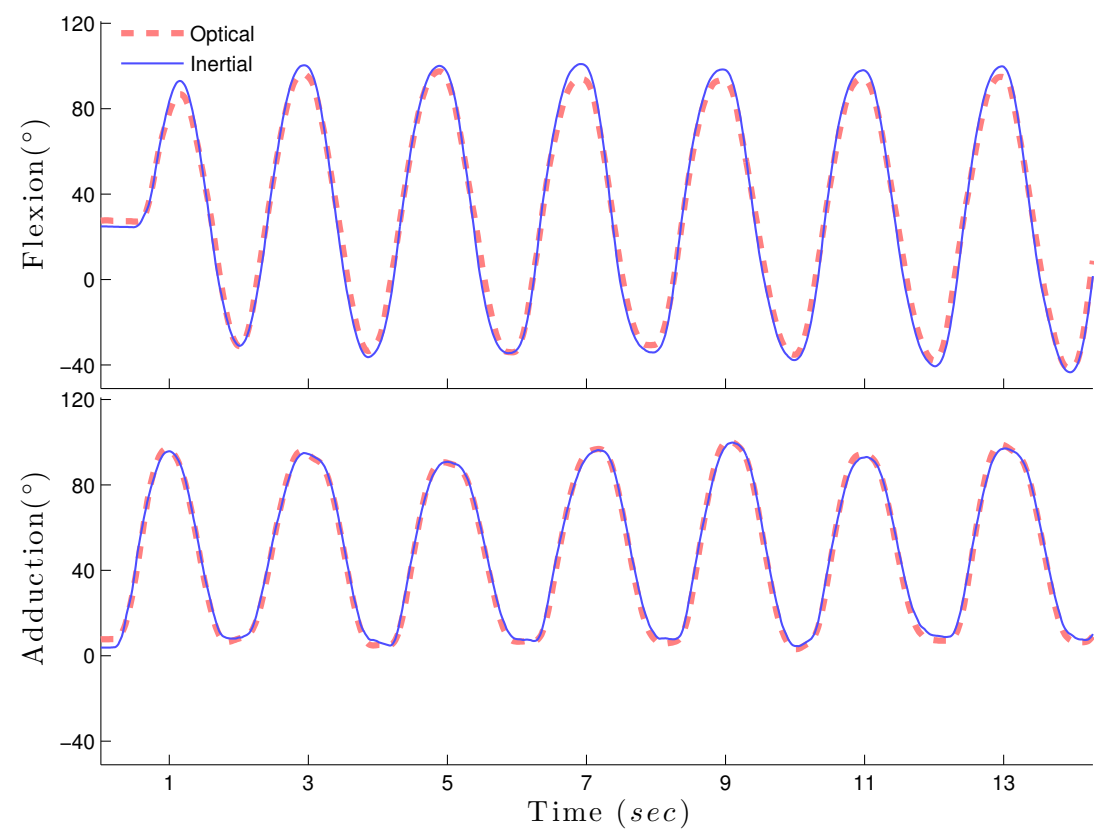

Figure 5.4: Shoulder angle estimates by the optical system (dashed red line) compared to inertial angles estimate (solid blue line).

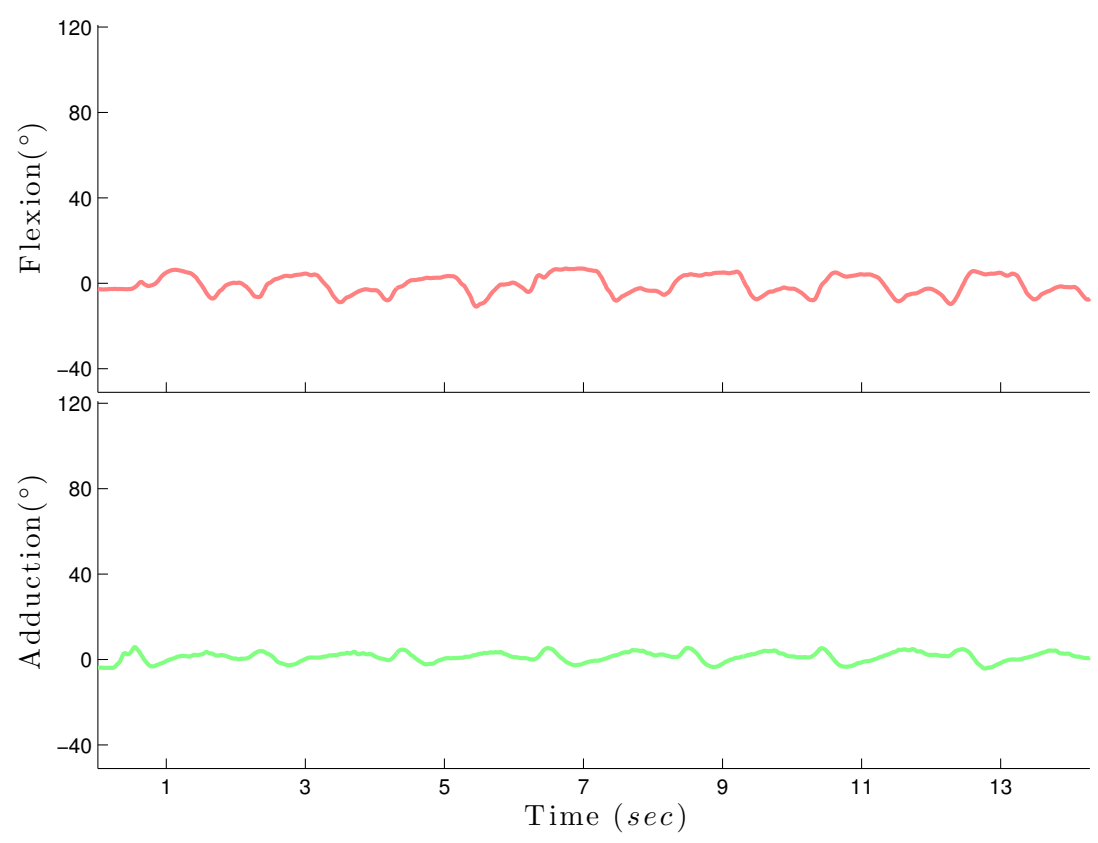

Figure 5.5: Difference between estimates using optical and inertial trackers. 
Fig. 5.4 shows shoulder angles during simple planar rotation at normal daily life movement rate. The top panel of the figure shows shoulder flexion/extension angle estimates using both inertial (solid blue line) and optical systems (dashed red line). The bottom panel shows the shoulder abduction/adduction angle estimates. The error between shoulder angle estimates from both tracking systems is shown in Fig. 5.5. Error is consistently small for both shoulder angles, and is slightly smaller than those of the elbow and forearm. Table 5.2 shows the average result for each task among subjects performing complex movements.

Table 5.2: Average correlation $r$, average RMSE and peak-to-peak average error between optical and inertial estimates of shoulder, elbow and forearm angles during prescribed activities at normal movement rate.

\begin{tabular}{lccc}
\hline \hline Task & $r$ & RMSE $\left(^{\circ}\right)$ & Peak Error $\left(^{\circ}\right)$ \\
\hline Touching nose & 0.94 & 6.5 & 9.8 \\
Reaching for a doorknob & 0.95 & 5.5 & 8.8 \\
\hline \hline
\end{tabular}

Fig. 5.6 shows elbow and shoulder flexion/extension angles obtained from the inertial and reference tracking systems for one of the subjects performing the task of repeated arm extension and flexion in order to touch the nose. The error between the two tracking systems is presented in Fig. 5.7. Both figures show that the difference between angle estimates from both systems, especially for shoulder flexion/extension, are slightly larger than what was obtained for the same angles during simple planar movement.

Subjects performed the same arm rotations described in Table 5.1 at a faster rotation rate of $420^{\circ} / \mathrm{s}$. Fig. 5.8 shows the estimated inertial shoulder angles compared to those obtained from the optical system. We obtained an average RMSE of less than $8^{\circ}$ and an average peak-to-peak error of less than $12^{\circ}$ among the 8 subjects for all tasks. The error between the two tracking systems is presented in Fig. 5.9. 


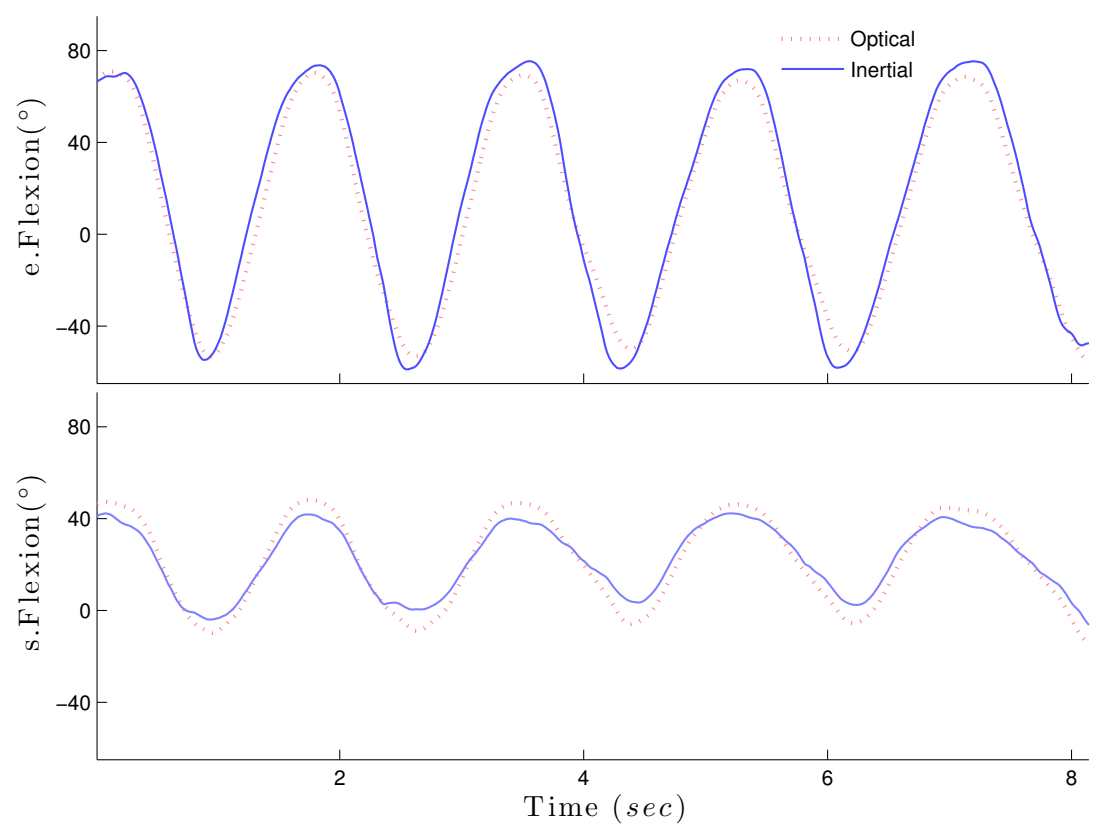

Figure 5.6: Elbow (top) and shoulder (bottom) flexion/extension angle estimates while subject repeatedly extends arm, then touches the nose with the index finger.

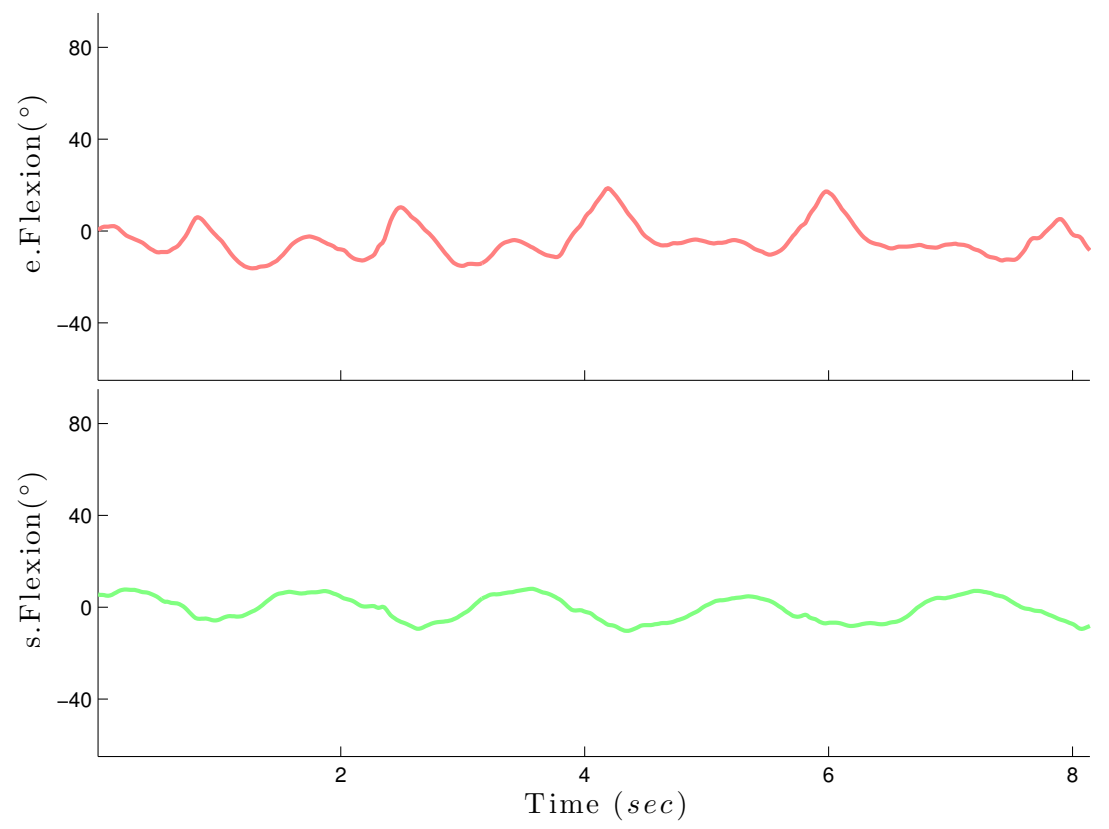

Figure 5.7: Estimate errors of Elbow (top) and shoulder (bottom) flexion/extension. 


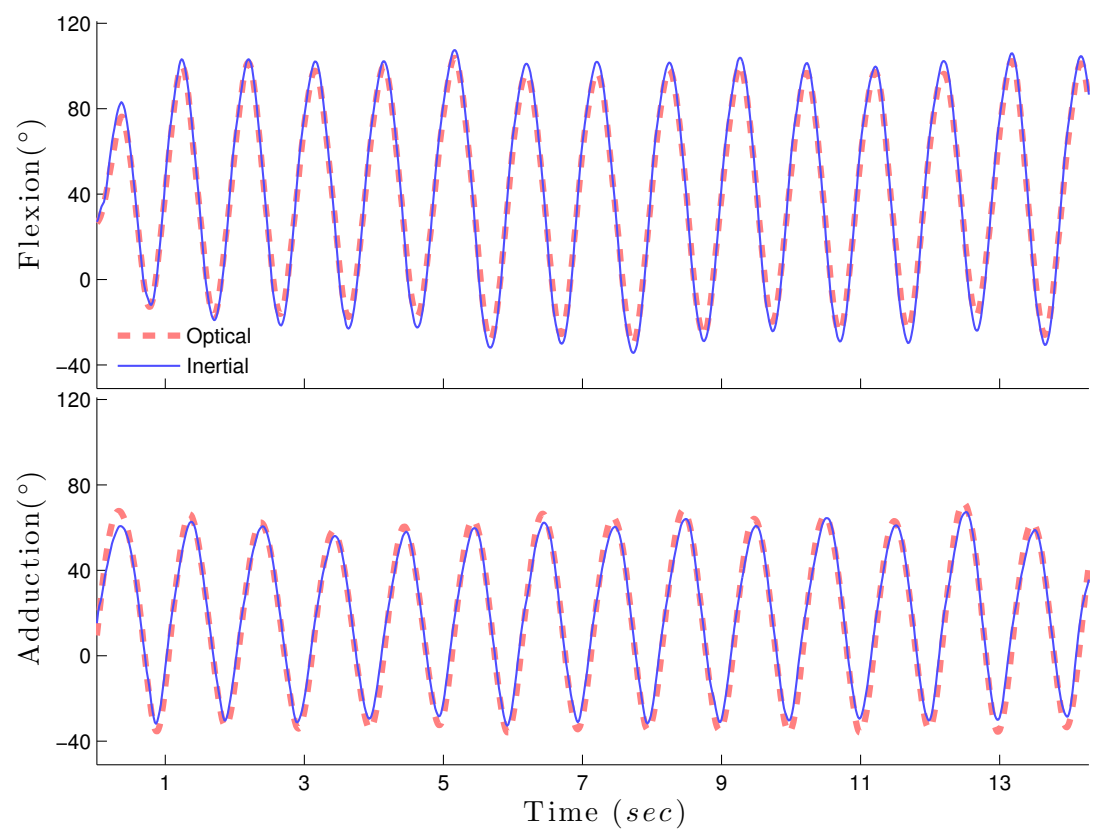

Figure 5.8: Shoulder flexion/extension and abduction/adduction angles during fast movement. Inertial (solid blue line) and optical angle estimates (dashed red line).

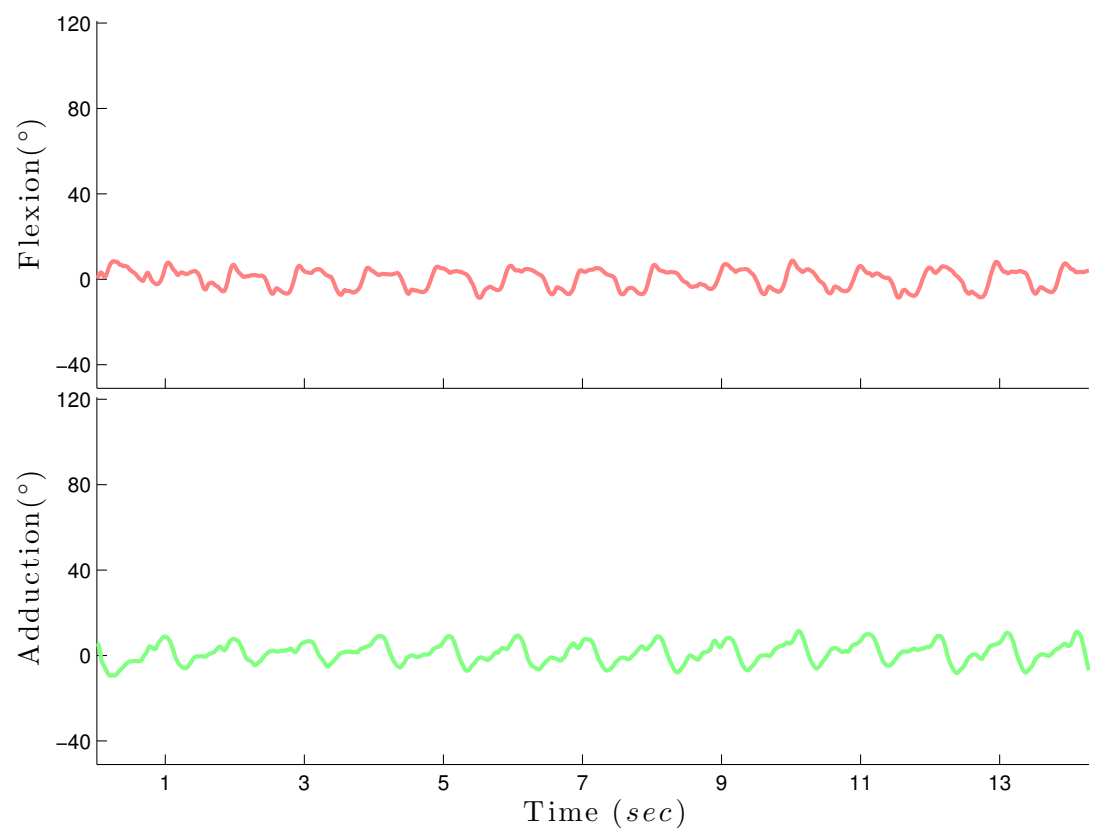

Figure 5.9: Difference between estimates using optical and inertial trackers. 


\subsection{Discussion}

We combined the kinematic models developed in Chapter 4 with the unscented Kalman filter to estimate shoulder, elbow and forearm angles. Two wireless wearable inertial measurement units were used to track human arm kinematics. Each measurement unit contained triaxial gyroscopes and accelerometers. We used an optical motion tracker as a reference system to assess the performance of our inertial tracker. We compared shoulder and elbow joint angles estimated by the inertial system to those estimated by the optical system. Two different data measurements from 8 subjects were used to evaluate the tracking performance. In the first data set, each subject performed 15 seconds of shoulder and elbow planar rotation at daily life movement speed with an average rotation rate of $180^{\circ} / \mathrm{s}$. Root mean squared error (RMSE) between angle estimates ranged from $4.4^{\circ}$ to $6.5^{\circ}$ with a correlation coefficient $r \geq 0.97$. This is a very reasonable error range compared to what was achieved by Bachmann et al. who reported an error range of $12^{\circ}-16^{\circ}$ [7].

Due to the modeling structure of measurement equations with kinematic chains, estimation accuracy of the distal angles are affected by the accuracy of the proximal angles [82]. In other words, error in shoulder angles may result in additional error in elbow angles. This might explain the slightly larger error in elbow and forearm angle estimates. Table 5.1 shows that maximum estimation error occurred at elbow flexion/extension and forearm supination/pronation angles.

Many of the inertial tracking studies discussed in previous chapters limited their performance assessment to slow movement. Contrary to these studies, we evaluated the performance of our algorithm in tracking fast movement with an average rotation rate of $420^{\circ} / \mathrm{s}$. The RMSE for the shoulder, elbow and forearm angles was less than $8^{\circ}$ on average, with correlation coefficient of $r \geq 0.95$.

Although tracking errors were minimal in most of the assessment results, performance accuracy might have been reduced by inertial sensor bias. Bias consists of a 
deterministic and a random parts. The deterministic bias refers to the offset in measurements even when they are stationary. The deterministic offset of the Gyroscopes was estimated from a three second stationary calibration period, and was removed from the gyroscope data before calculating the angles. The random bias or drift refers to the rate at which inertial sensor error accumulates with time. Gyroscope and accelerometer random drift can be estimated when modeled as a random process in the system equations. However, this increases the process model dimension by adding 6 more states for each measurement unit. This in turn, increases the computational cost of the tracker. The effect of modeling sensor drift will be analyzed in Chapter 6.

Some of the estimation error might also be attributed to markers moving independently of each other, especially during fast movement. Marker placement over anatomical landmarks can create skin artifacts [25]. The motion of the skin-mounted markers is a major source of error in the kinematic measurement of human movement. Another common problem in motion capture is marker occlusion. When a significant proportion of marker data was missing in any of the recordings, the entire recording had to be discarded. In addition to an extensive set-up time, a few recordings were stopped and had to be repeated to ensure that all markers were visible by the cameras. Six of 56 recordings were discarded due to missing marker data. Vicon optical data was sampled at $60 \mathrm{~Hz}$. When one or two markers were non-visible for 6 frames or less, the occluded marker positions were estimated using interpolation.

Our tracking algorithm utilizes both translational and gravitational components of acceleration. This results in accurate tracking performance during fast and slow movements. However, one of the limitations of this study and of all of tracking algorithms discussed in the introduction, is the use of short movement for performance assessment. Although we used longer periods than most other studies, our continuos recordings lasted only 2 minutes for 4 of the 8 subjects. In the next chapter, we 
use long recordings of 15 minutes with a second reference system to study the effect of modifying the kinematic model. To reduce the effect of sensor drift on the estimated angles, we use a modified model in Chapter 6. The model incorporates prior knowledge of physical constraints and human natural range of motion, as well as a gyroscope and accelerometer drift model. The combined effect of imposing physical constraints and modeling the sensor drift is investigated in the next chapter. 


\section{Modified Arm Model}

One of the major sources of error in estimating body kinematics using inertial sensors is the inherent bias and random drift of accelerometers and gyroscopes. Bias is a constant sensor output when it is not undergoing any movement. When integrated, bias causes a position or orientation error that grows with time. The constant bias can easily be estimated and subtracted from the sensor output. This is achieved by recording a few seconds while sensors are stationary, then averaging and subtracting that constant bias.

Random drift is a more critical source of error in accelerometers and gyroscopes. When integrated, this random drift introduces angle error that grows proportionally with time. In Chapter 2, we introduced some of the algorithms that were developed in attempt to reduce the effect of sensor drift on the accuracy of angle estimates. The most common approach is to use sensor fusion, where additional sensors such as magnetometers are used to update and correct the estimated angles. However, we showed in Section 2.1 that these additional sensors have their own drawbacks and the systems are only applicable under limited circumstances. Other tracking algorithms utilize domain knowledge about human movement to reduce the effect of drift by restricting estimates within an acceptable range. Another approach to reducing the effect of sensor drift is the use of zero-velocity updates. This technique has been successfully used in gait analysis and pedestrian navigation studies, where stationary stance periods are detected and used to reset the angular rate to zero [113].

In this chapter, we introduce modified process and measurement models to mit- 
igate the effect of sensor drift and to increase the tracking accuracy. The modified tracker incorporates gyroscope and accelerometer random drift models in Section 6.2, utilizes physical constraints and human natural range of motion in Section 6.3, and applies zero-velocity updates to provide periodic correction to the error in angle estimates in Section 6.4. Effect of each individual modification is analyzed separately and compared to baseline performance results of Section 6.1.1. The three techniques are then combined in one algorithm and evaluated in tracking joint angles during long movement periods in Section 6.5. We quantify the performance of our UKF-based algorithm by comparing the angle estimates to those obtained directly form a 6-axis high-precision industrial robot arm during 15-minute recordings of slow, medium and fast-speed movement.

\subsection{Robot Arm Joint Angles}

To evaluate the performance of the modified inertial tracking system, we use an industrial Epson C3 robot arm (Epson Robots, Carson, California) with six degrees of freedom. The robot arm is a high speed, and a very high precision industrial robot that is used for assembly of medical devices and parts. Three Opal sensors (APDM, Portland, OR), each containing triaxial accelerometers and gyroscopes, are placed on the upper arm, forearm and wrist of the robot as shown in Fig. 6.1. Table 6.1 shows the Epson $\mathrm{C} 3$ range of motion and operating speed of the six joints. Inertial sensor data and robot data were synchronized by calculating the lag time using cross-correlation analysis.

Typically, the human shoulder joint is modeled as a ball-and-socket joint with three degrees of freedom (DOFs). However, for the purpose of quantifying the performance of our algorithm, we model the shoulder with only two DOFs to match those of the industrial robot used in this chapter for comparison. Fig. 6.2 shows the arm model with the stationary base reference frame 0 at the center of the shoulder 
Table 6.1: Maximum operating speed and motion range for the robot arm.

\begin{tabular}{lcc}
\hline \hline Task & Rate & Max. Motion Range \\
\hline Shoulder Internal/External Rotation & $450^{\circ} / \mathrm{s}$ & $\pm 180^{\circ}$ \\
Shoulder Flexion/Extension & $450 \% / \mathrm{s}$ & $-160^{\circ} \sim+65^{\circ}$ \\
Elbow Flexion/Extension & $514 \% / \mathrm{s}$ & $-51^{\circ} \sim+225^{\circ}$ \\
Forearm Supination/Pronation & $553 \% / \mathrm{s}$ & $\pm 200^{\circ}$ \\
Wrist Flexion/Extension & $553^{\circ} / \mathrm{s}$ & $\pm 135^{\circ}$ \\
Wrist Twist & $720^{\circ} / \mathrm{s}$ & $\pm 360^{\circ}$ \\
\hline \hline
\end{tabular}

joint. Frame 1 represents shoulder internal/external rotation, and frame 2 represents shoulder flexion/extension. The robot elbow joint is a hinge joint that allows movement in one plane, flexion/extension, represented by frame 3 . The fourth joint is a pivot joint that allows for the forearm pronation/supination, and is represented by frame 4. Frames 5 and 6 represent wrist flexion/extension, and wrist twist respectively.

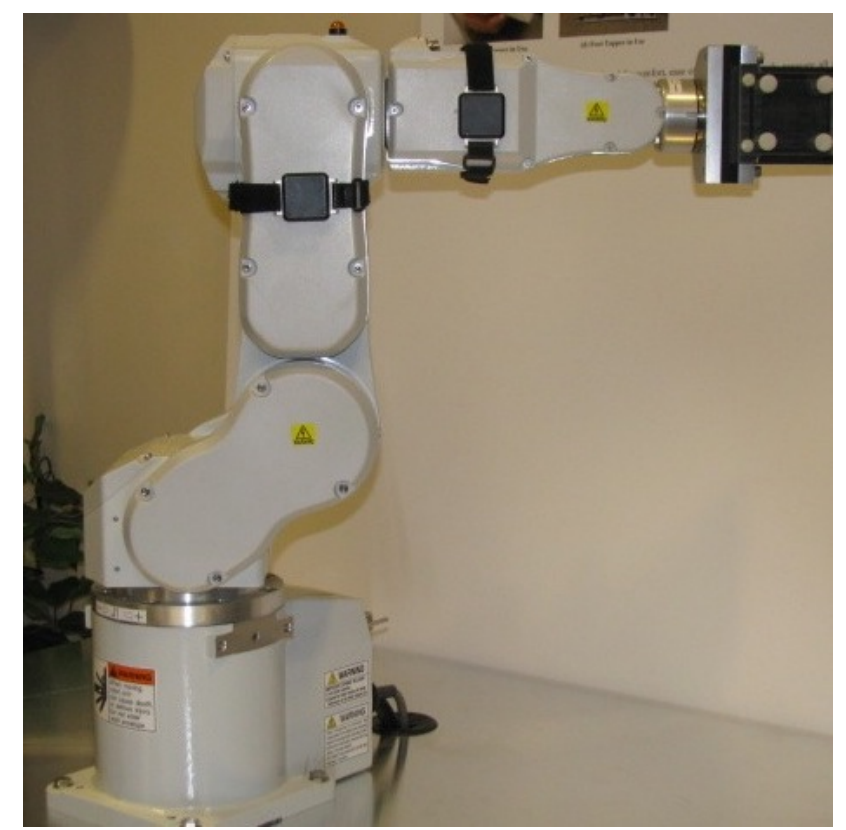

Figure 6.1: Opal inertial sensors placement on Epson C3 robot arm.

Table 6.2 shows the $\mathrm{D}-\mathrm{H}$ parameters, where $\alpha_{i-1}$ is the angle to rotate to make 


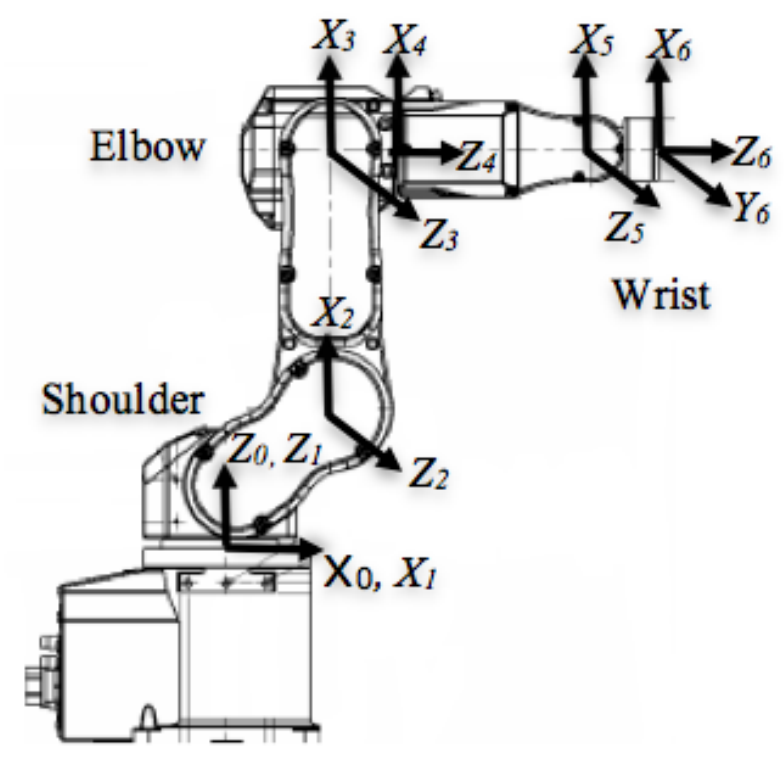

Figure 6.2: Diagram of the arm model with Frame 0 as the static reference at the base. Frames 1 and 2 represent shoulder internal/external rotation, and flexion/extension. Frame 3 represents elbow flexion/extension. Frame 4 represents forearm pronation/supination. Wrist flexion/extension, and wrist twist are represented by frames 5 and 6 respectively.

the two coordinate systems coincide, $l_{\mathrm{u}}$ is the length of the upper arm, $a_{i}$ is the link length between joint axis $Z_{i}$ and $Z_{i+1}$ along $X_{i}$, and $\theta_{i}$ is the $i^{\text {th }}$ angle of rotation.

Table 6.2: Denavit-Hartenberg parameters for the 6 DOFs arm model.

\begin{tabular}{ccccc}
\hline \hline Frame & $\alpha_{i-1}$ & $a_{i-1}$ & $d_{i}$ & $\theta_{i}$ \\
\hline 1 & 0 & 0 & 0 & $\theta_{1}$ \\
2 & $\pi / 2$ & $a_{1}$ & 0 & $\theta_{2}+\pi / 2$ \\
3 & 0 & $l_{\mathrm{u}}$ & 0 & $\theta_{3}$ \\
4 & $\pi / 2$ & 0 & 0 & $\theta_{4}$ \\
5 & $-\pi / 2$ & 0 & 0 & $\theta_{5}$ \\
6 & $\pi / 2$ & 0 & 0 & $\theta_{6}$ \\
\hline \hline
\end{tabular}




\subsubsection{Baseline Performance Results}

In this section, we present baseline performance results that will be used to assess the performance improvement introduced by employing the drift reduction techniques. Planar and complex robot arm movements were collected in three data sets at three different rotational rates. Each data set lasted at least 15 minutes. Each recording started and ended with a stationary period of 3 seconds at the initial pose. This period was used to estimate the gyroscope deterministic bias offset. The stationary gyroscope average measurement was removed before calculating the angles [122]. The rest of each recording was designed to include simple planar rotation about each of the six joints, followed by one second of a stationary period, and ending with more complex movements involving shoulder, elbow and wrist joints simultaneously. This arm trajectory was repeated a few times to obtain a continuous 15-minute recording of robot arm movement to closely mimic human arm movement performing daily activities at different speeds.

Firstly, we introduce tracking results for the data set of the arm movement at slow speed. We define robot slow speed as one fourth of the arm maximum rotational rate shown in Table 6.1. Slow rotation rate for each joint is show in Table 6.3.

Table 6.3: Average rotational rate, correlation $r$, and RMSE in angle estimates.

\begin{tabular}{lccr}
\hline \hline Task & $\operatorname{Rate}\left({ }^{\circ} / \mathrm{s}\right)$ & $r$ & $\operatorname{RMSE}\left({ }^{\circ}\right)$ \\
\hline Shoulder Internal/External Rotation & 112 & 0.92 & 25.0 \\
Shoulder Flexion/Extension & 112 & 1.00 & 1.1 \\
Elbow Flexion/Extension & 128 & 1.00 & 1.1 \\
Forearm Supination/Pronation & 138 & 1.00 & 1.4 \\
Wrist Flexion/Extension & 138 & 1.00 & 1.2 \\
Wrist Twist & 180 & 1.00 & 1.8 \\
\hline \hline
\end{tabular}

Fig. 6.3 shows an example of the gyroscope sensor unit placed on the wrist. Fig. 6.5 and 6.6 show the last two minutes of elbow and wrist true angles and their estimates. Estimation error for the entire recording is shown in Fig. 6.7 and 6.8 . 

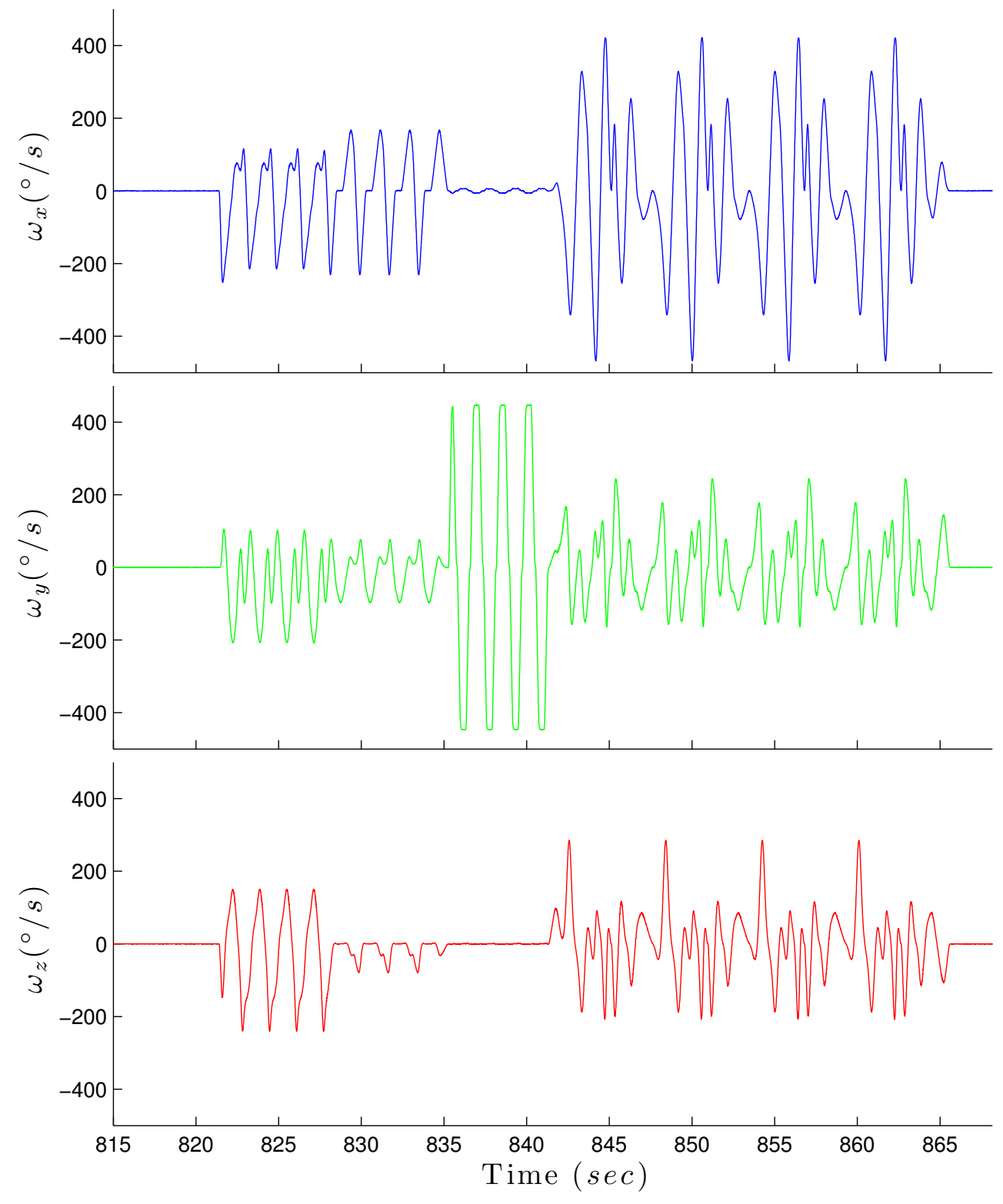

Figure 6.3: Rotational rate data during the last minute from the gyroscope sensor unit placed on the wrist. 


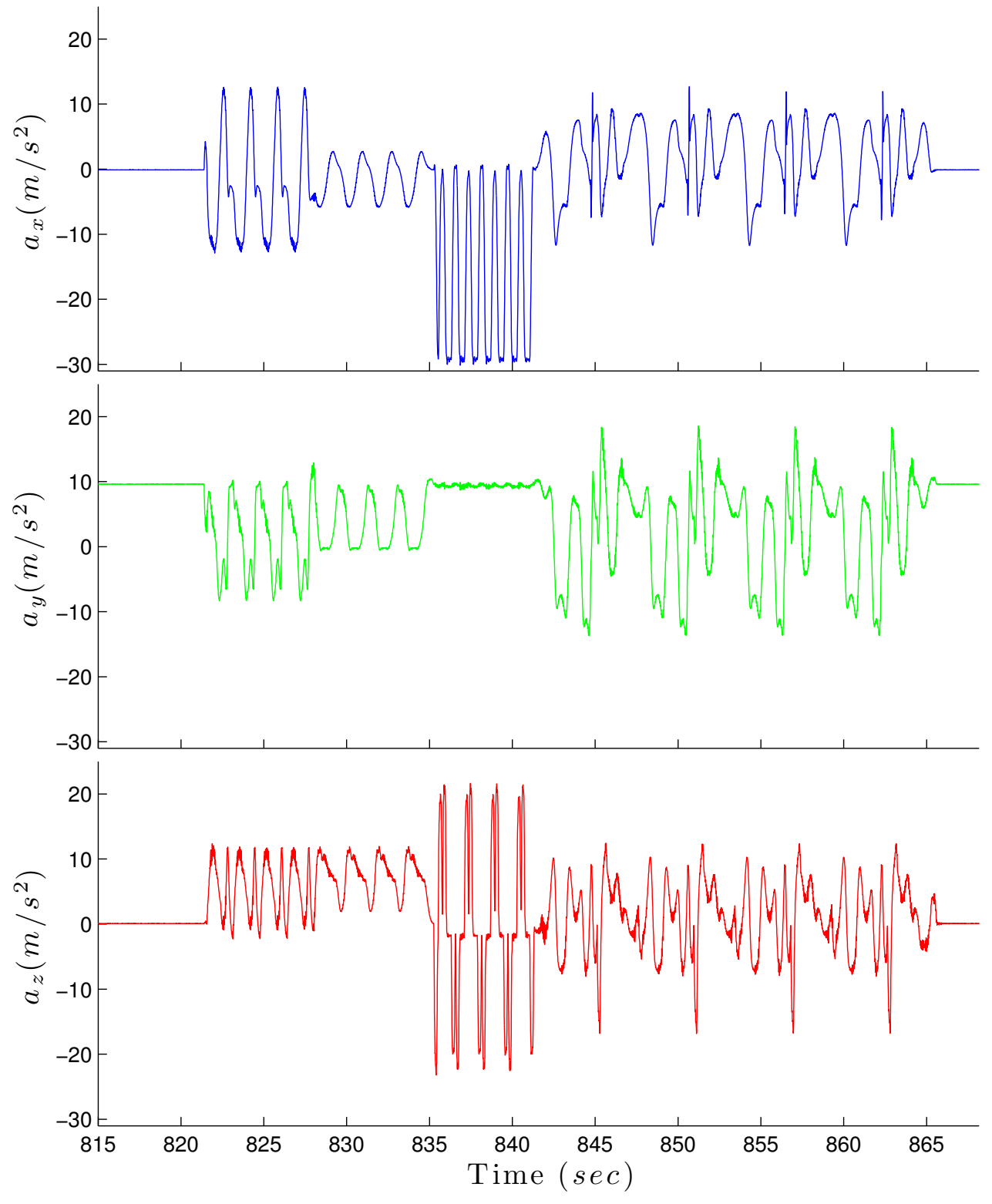

Figure 6.4: Acceleration data during the last minute from the sensor unit placed on the wrist. 


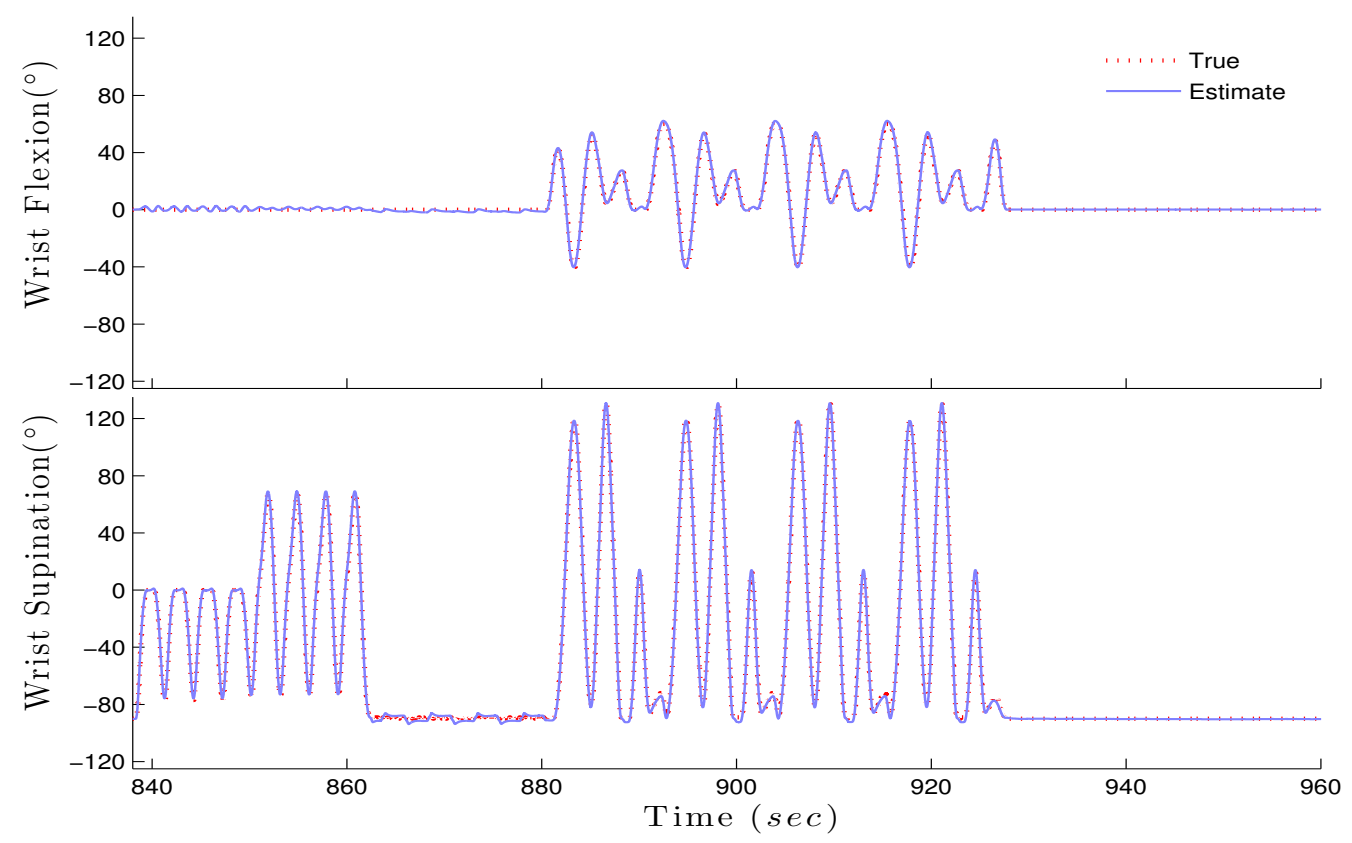

Figure 6.5: Wrist angles during the last 2 minutes at an average rotation rate of $160^{\circ} / \mathrm{s}$.

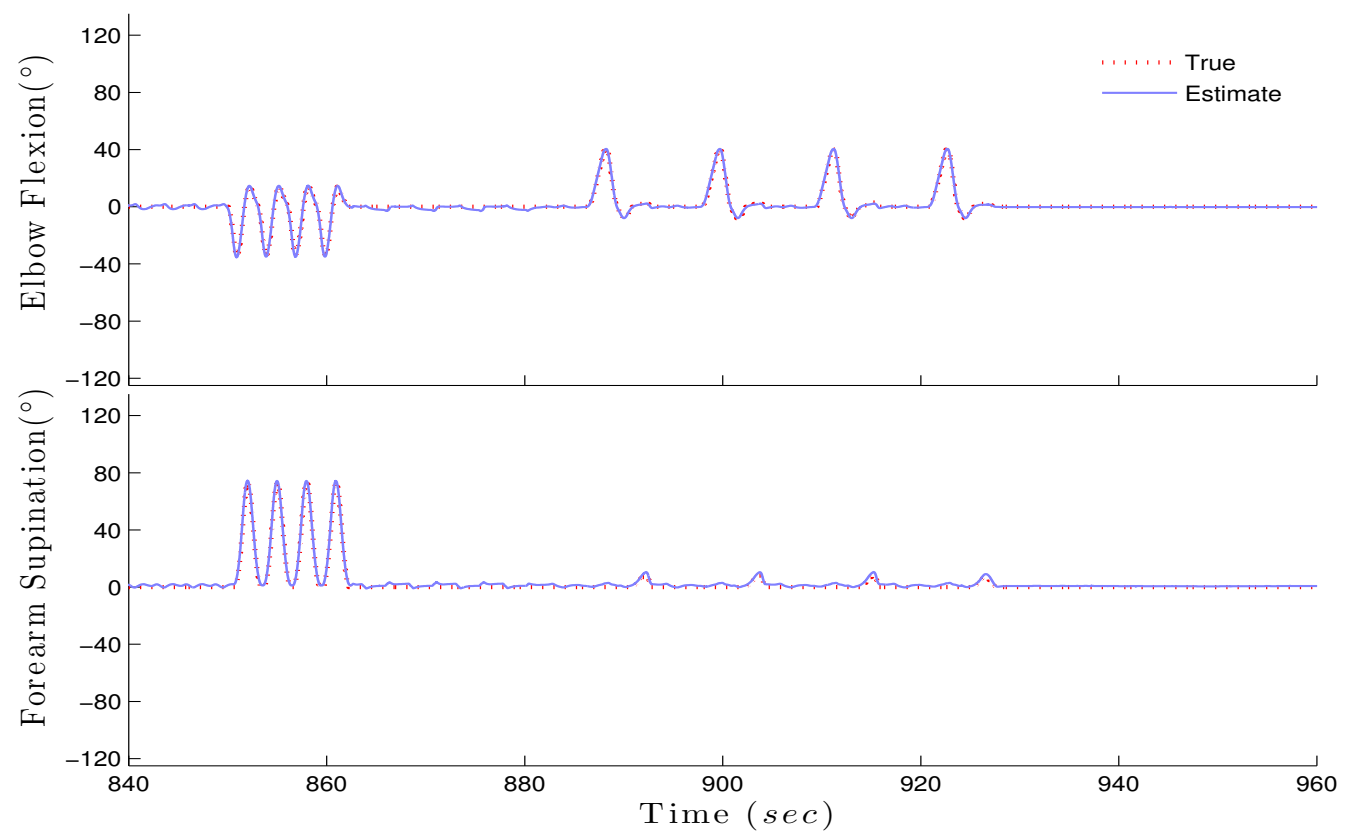

Figure 6.6: Elbow angles during the last 2 minutes at an average rotation rate of $130 \% \mathrm{~s}$. 


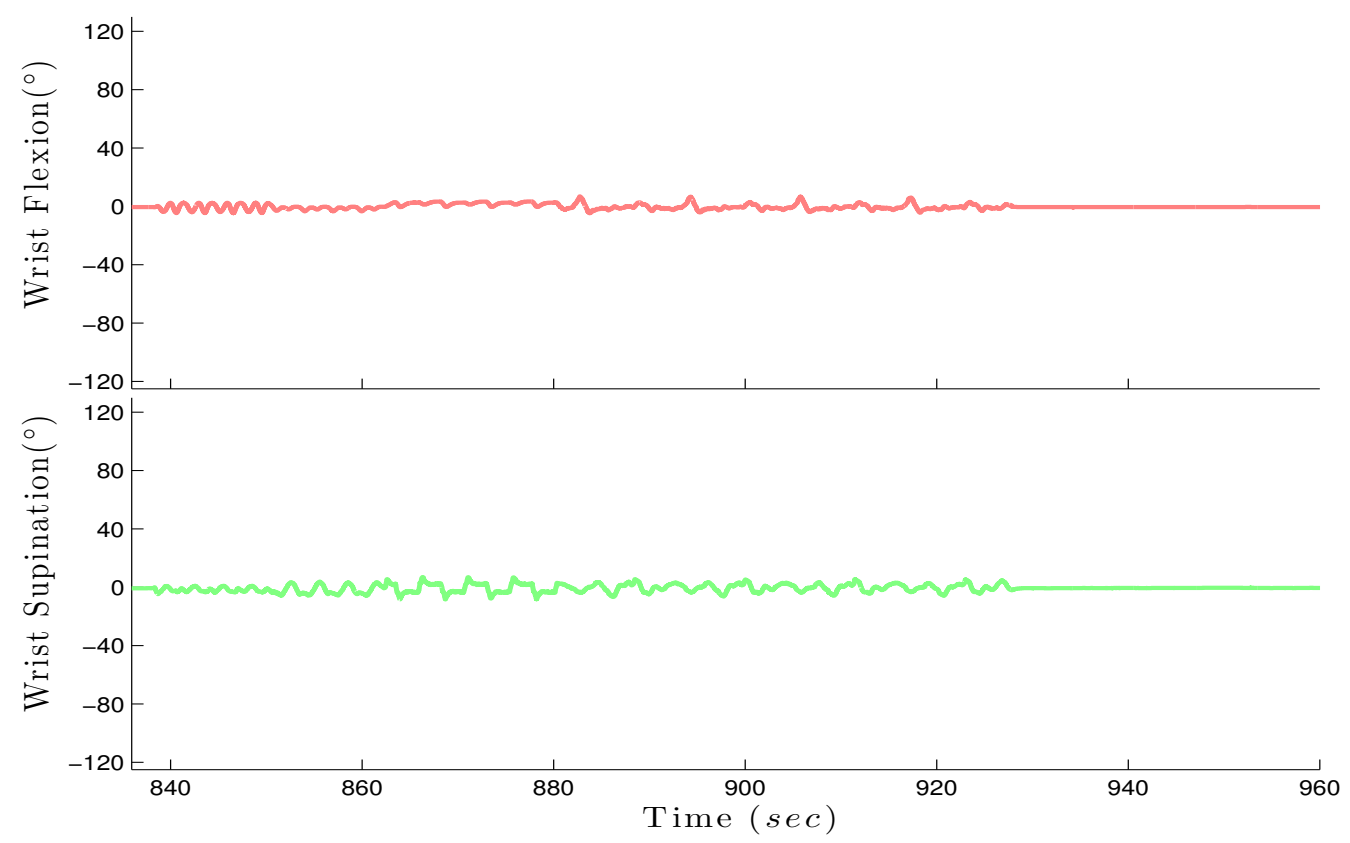

Figure 6.7: Wrist joint angle error between true and inertial estimates.

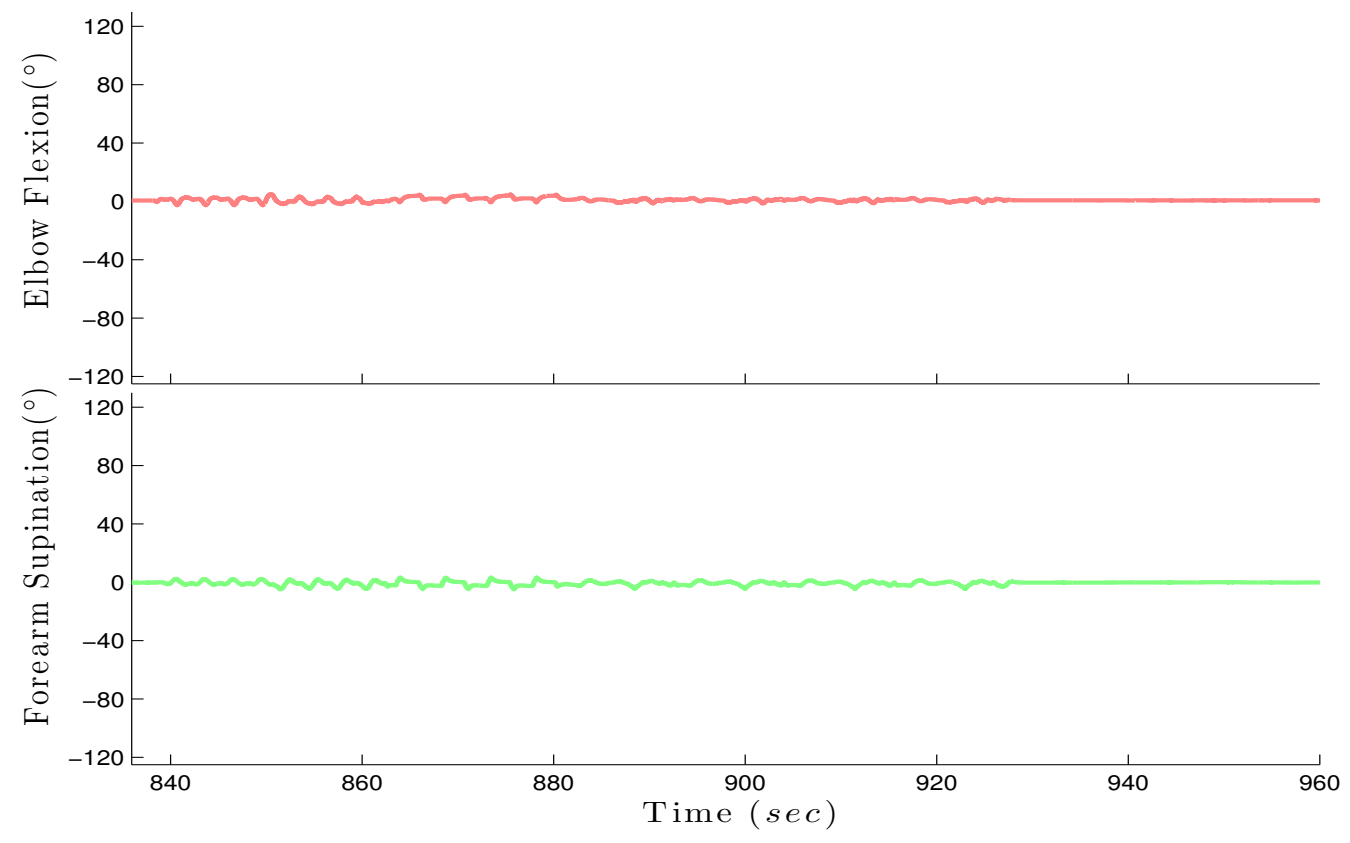

Figure 6.8: Elbow joint angle error between true and inertial estimates. 


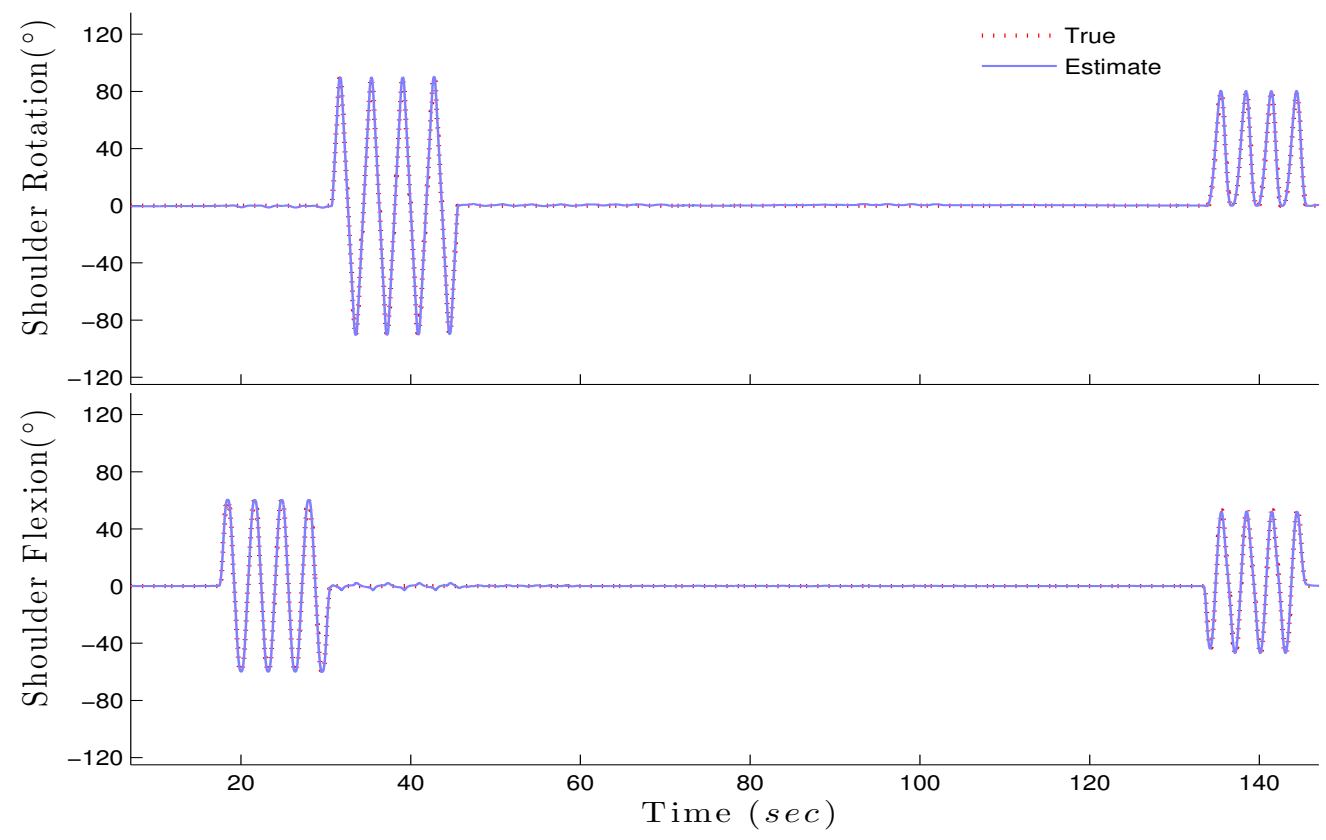

Figure 6.9: Shoulder angles during the first 2 minutes at an average rotation rate of $112 \% \mathrm{~s}$.

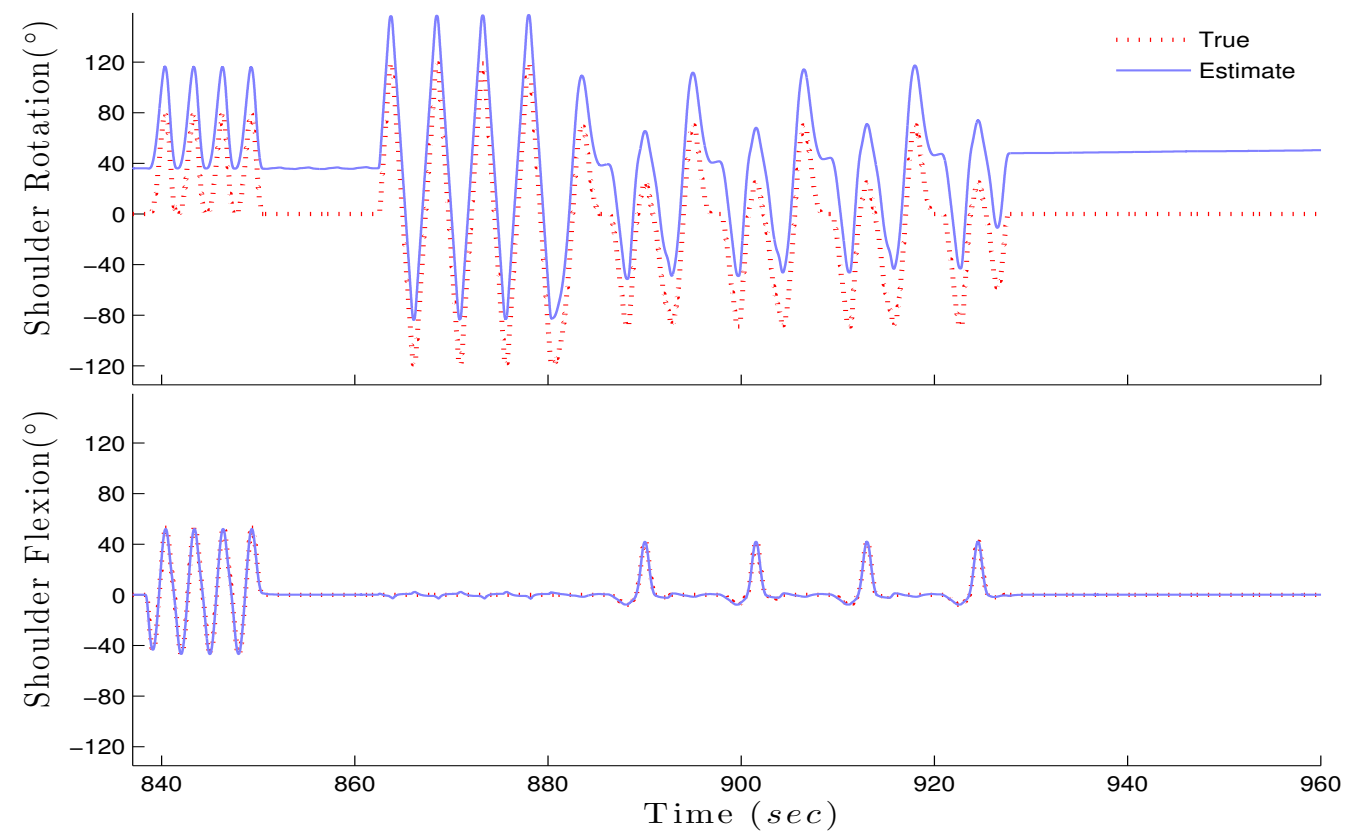

Figure 6.10: Shoulder angles during the last 2 minutes at an average rotation rate of $112 \% \mathrm{~s}$. 
Fig. 6.9 and 6.10 show the first and last two minutes of shoulder joint angles, respectively. Table 6.3 shows a comparison between the true robot joint angles and their estimates using the inertial tracker. A perfect correlation and very small error between inertial estimates and the true robot wrist and elbow joint angles were obtained during planar and more complex movement at the slow rotation rate. Similarly, shoulder flexion/extension angle estimates perfectly matched the true robot angles. On the other hand, Fig. 6.10 shows the effect of sensor random drift around the vertical axis, with an increased heading error after 15 minutes, compared to the negligible heading error during the first 2 minutes in Fig. 6.9.

Second and third data sets were collected of the arm movement at medium and fast rotational speed, which were defined as one half and full range of the maximum rotational rate shown in Table 6.1. That is, a rotational rate of $225-360^{\circ} / \mathrm{s}$ during medium speed, and a rate range of $450-720^{\circ} / \mathrm{s}$ during fast movement. We obtained a correlation coefficient $r \geq 0.98$ for all six joint angles. Consistent with the results obtained for movement during slow rotation, estimation errors between inertial estimates and true robot joint angles were less than $4^{\circ}$. Error in shoulder rotation estimates was still higher than in other join angles, although it dropped from $25^{\circ}$ to less than $10^{\circ}$. Table 6.4 shows tracking results during medium and fast rotation.

Table 6.4: RMSE between inertial and true robot angles during medium and fast rotation.

\begin{tabular}{lcr}
\hline \hline Task & Medium $\left(^{\circ}\right)$ & Fast $\left(^{\circ}\right)$ \\
\hline Shoulder Internal/External Rotation & 8.1 & 9.6 \\
Shoulder Flexion/Extension & 2.4 & 2.5 \\
Elbow Flexion/Extension & 2.6 & 3.3 \\
Forearm Supination/Pronation & 2.1 & 2.4 \\
Wrist Flexion/Extension & 2.2 & 2.9 \\
Wrist Twist & 3.9 & 3.8 \\
\hline \hline
\end{tabular}

Fig. 6.11 and 6.12 show the last two minutes of elbow and wrist movements during medium speed. Fig. 6.13 and 6.14 show first and last two minutes of shoulder 
angles.
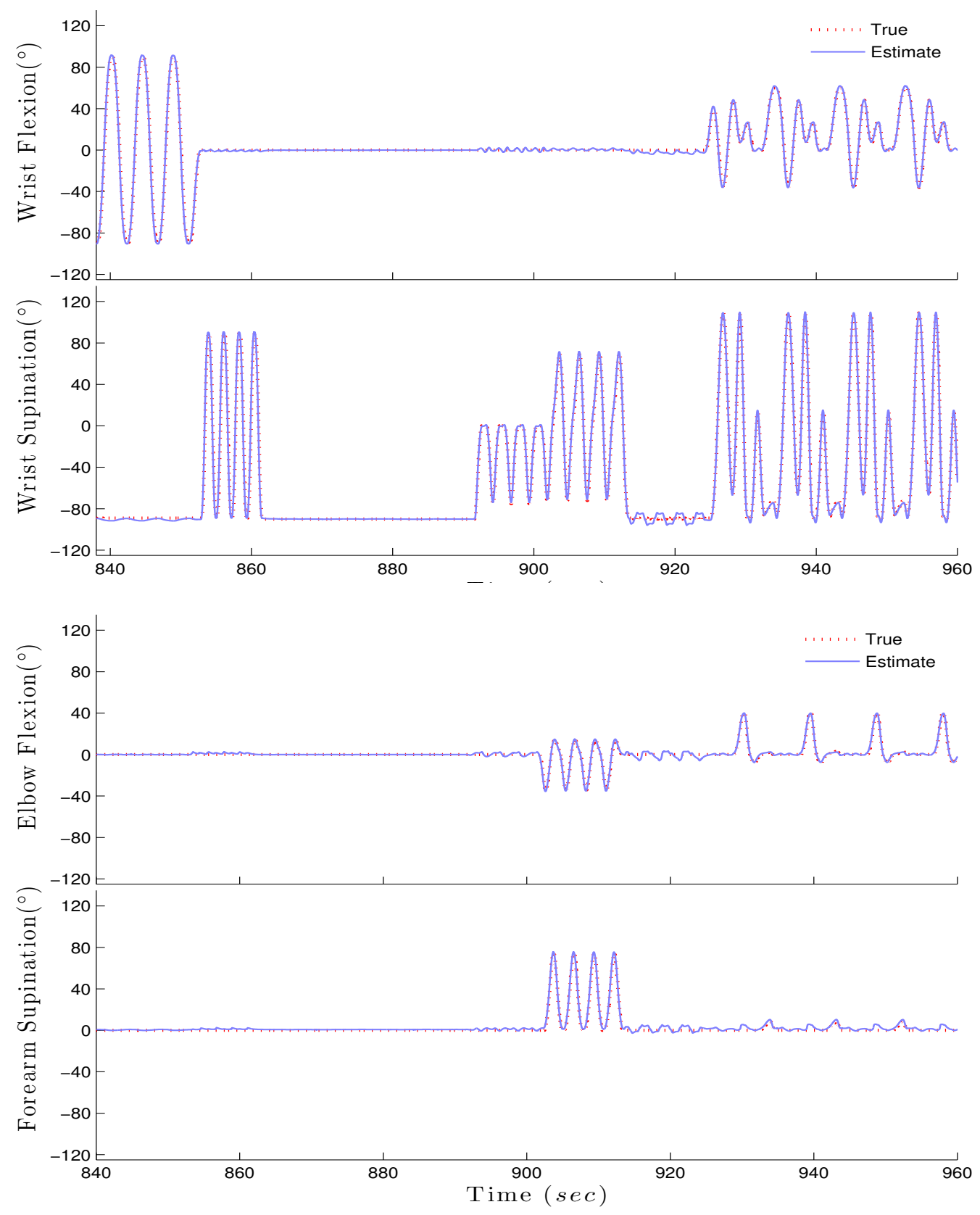

Figure 6.12: Elbow angles during the last 2 minutes at an average rotation rate of $260^{\circ} / \mathrm{s}$. 


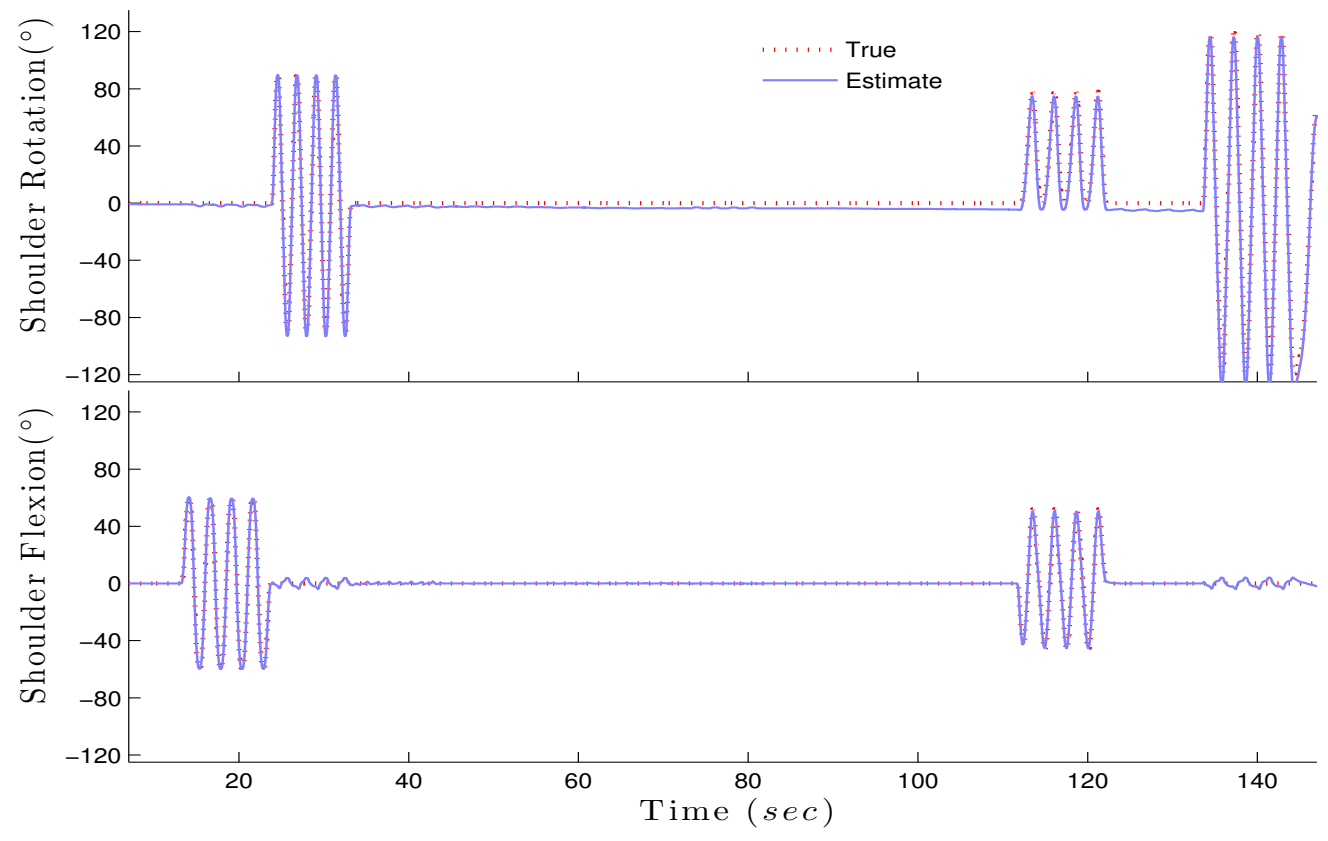

Figure 6.13: Shoulder angles during the first 2 minutes at a rotation rate of $225^{\circ} / \mathrm{s}$.

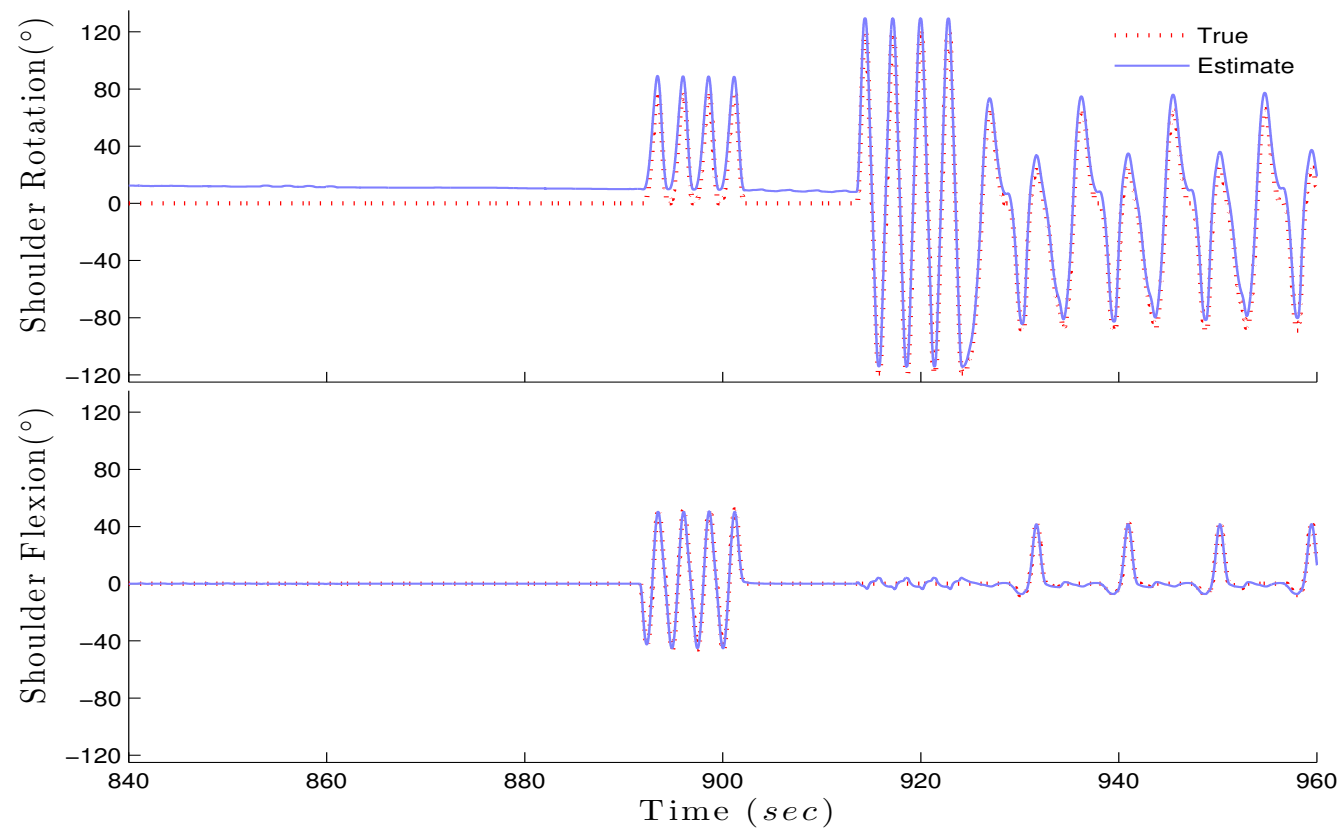

Figure 6.14: Shoulder angles during the last 2 minutes at a rotation rate of $225 \% \mathrm{~s}$. 


\subsection{Modeling Sensor Random Drift}

In this section, we modify the process model to include accelerometer and gyroscope sensors random bias. To reduce the effect of random drift on shoulder rotation angle estimates, we model the bias of the sensors of the IMU placed on the shoulder. Bias is modeled as a random walk, adding 6 more dimensions to the process model:

$$
\begin{aligned}
\theta_{i}(n+1) & =\theta_{i}(n)+T_{\mathrm{s}} \dot{\theta}_{i}(n)+\frac{1}{2} T_{\mathrm{s}}^{2} \ddot{\theta}_{i}(n) \\
\dot{\theta}_{i}(n+1) & =\dot{\theta}_{i}(n)+T_{\mathrm{s}} \ddot{\theta}_{i}(n) \\
\ddot{\theta}_{i}(n+1) & =\alpha \ddot{\theta}_{i}(n)+u_{\ddot{\theta}_{i}}(n) \\
\vdots & \\
\mathbf{b}_{\omega}(n+1) & =\mathbf{b}_{\omega}(n)+\mathbf{u}_{\mathbf{b} \omega}(n) \\
\mathbf{b}_{\mathbf{a}}(n+1) & =\mathbf{b}_{\mathbf{a}}(n)+\mathbf{u}_{\mathbf{b a}}(n)
\end{aligned}
$$

The 3D gyroscope bias $\mathbf{b}_{\omega}$ and $3 \mathrm{D}$ accelerometer bias $\mathbf{b}_{\mathbf{a}}$ are random walk with zero-mean white noise $\mathbf{u}_{\mathbf{b} \omega}$ and $\mathbf{u}_{\mathrm{ba}}$. The observation equation for the inertial measurement unit placed on the upper arm is given below:

$$
\begin{aligned}
& \dot{\omega}_{x}=\dot{\theta}_{1} \cos \left(\theta_{2}\right)+b_{\omega x} \\
& \dot{\omega}_{y}=-\dot{\theta}_{1} \sin \left(\theta_{2}\right)+b_{\omega y} \\
& \dot{\omega}_{z}=\dot{\theta}_{2}+b_{\omega z} \\
& \dot{v}_{x}=a_{1} \sin \left(\theta_{2}\right) \dot{\theta}_{1}^{2}+g \cos \left(\theta_{2}\right)-\dot{\theta}_{1}{ }^{2} a_{2} \sin \left(\theta_{2}\right)^{2}-\dot{\theta}_{2}{ }^{2} a_{2}+b_{a x} \\
& \dot{v}_{y}=a_{1} \cos \left(\theta_{2}\right) \dot{\theta}_{1}{ }^{2}-g \sin \left(\theta_{2}\right)-\dot{\theta}_{1}{ }^{2} a_{2} \cos \left(\theta_{2}\right) \sin \left(\theta_{2}\right)+\ddot{\theta}_{2} a_{2}+b_{a y} \\
& \dot{v}_{z}=a_{1} a_{2} \cos \left(\theta_{2}\right) \dot{\theta}_{1} \dot{\theta}_{2}+\ddot{\theta}_{1} a_{2} \sin \left(\theta_{2}\right)+\dot{\theta}_{1} \dot{\theta}_{2} a_{2} \cos \left(\theta_{2}\right)-a_{1} \ddot{\theta}_{1}+b_{a z}
\end{aligned}
$$

where $\left(\omega_{x}, \omega_{y}, \omega_{z}\right)$ are the triaxial gyroscope data, and $\left(\dot{v}_{x}, \dot{v}_{y}, \dot{v}_{z}\right)$ are the triaxial accelerometer data at time $n, \theta_{i}(n)$ is the $i^{t h}$ angle at time $n, \dot{\theta}_{i}$ is the angular velocity, and $\ddot{\theta}_{i}$ is the angular acceleration. The distance between elbow flexion joint and the device is $a_{2}$. The time index $n$ was dropped from the measurement equations for ease 
of readability. Observation equations for the forearm and wrist sensors are too long to be shown here.

\subsubsection{Results}

Table 6.5 shows a comparison between the true robot joint angles and their estimates using the inertial tracker with the modified kinematic equations. The table also includes baseline RMSE during slow arm movement for comparison purposes. Results show a very small errors between inertial estimates and true robot wrist and elbow joint angles. Similarly, shoulder flexion and extension angle estimates perfectly matched the true robot angles. The RMSE of the shoulder internal/external rotation angle dropped from $25.0^{\circ}$ to $5.2^{\circ}$.

Table 6.5: RMSE of inertial angle estimates using the modified dynamic equations with bias estimates, compared to baseline results of angles during slow rotation.

\begin{tabular}{lcr}
\hline \hline Task & $\operatorname{RMSE}\left({ }^{\circ}\right)$ & Baseline RMSE $\left(^{\circ}\right)$ \\
\hline Shoulder Internal/External Rotation & 5.2 & 25.0 \\
Shoulder Flexion/Extension & 0.9 & 1.1 \\
Elbow Flexion/Extension & 1.1 & 1.1 \\
Forearm Supination/Pronation & 1.4 & 1.4 \\
Wrist Flexion/Extension & 1.3 & 1.2 \\
Wrist Twist & 1.8 & 1.8 \\
\hline \hline
\end{tabular}

Fig. 6.15 shows the last two minutes of a 15 minute recording of simple planar and more complex robot shoulder joint angles. Shoulder angle estimation error of the last two minutes is shown in Fig. 6.16. Both figures and results in Table 6.5 show that the new model dramatically decreased shoulder internal/external rotation angle errors from $25.0^{\circ}$ to $5.2^{\circ}$, with an increased correlation from 0.92 to 0.99 . 


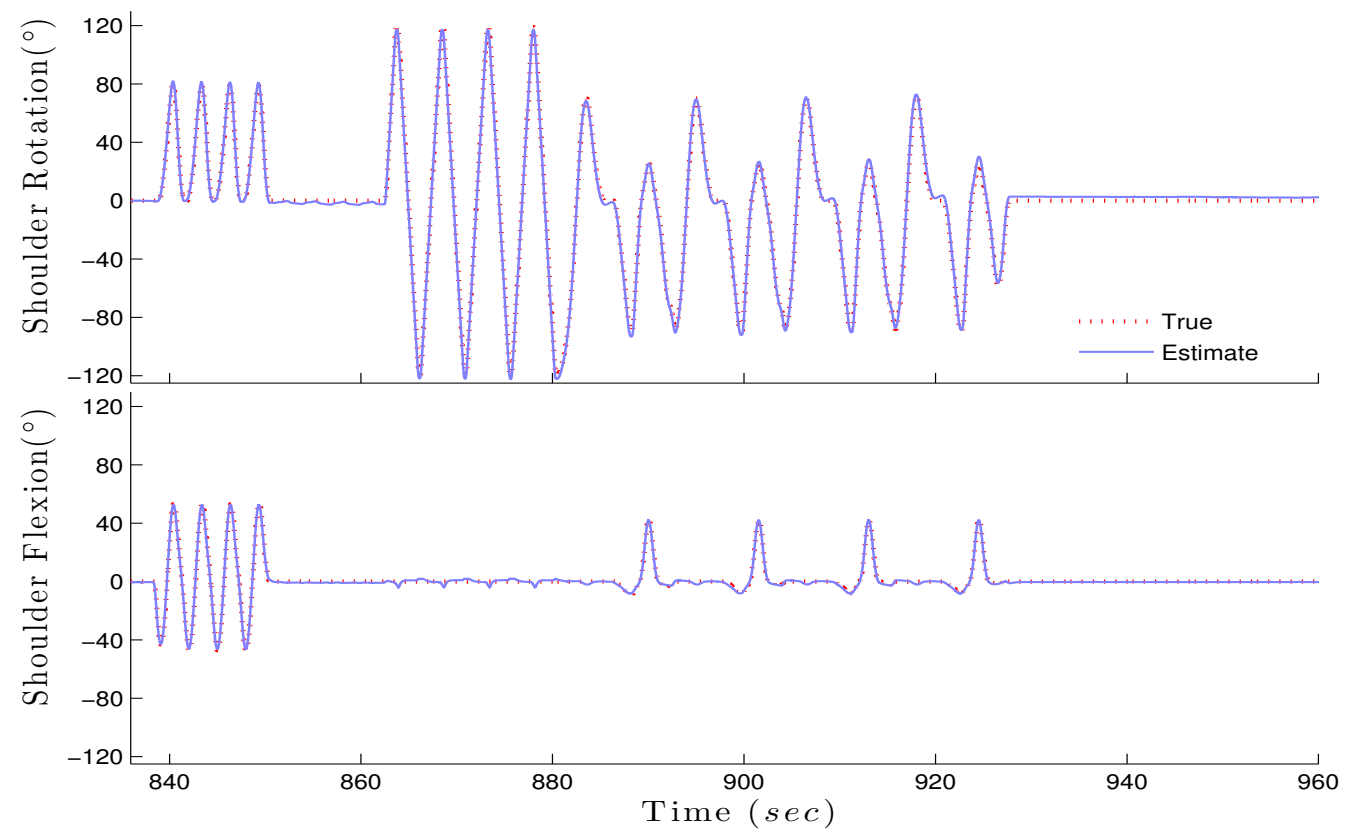

Figure 6.15: Shoulder angles during the last two minutes at a rotation rate of $112 \% \mathrm{~s}$.

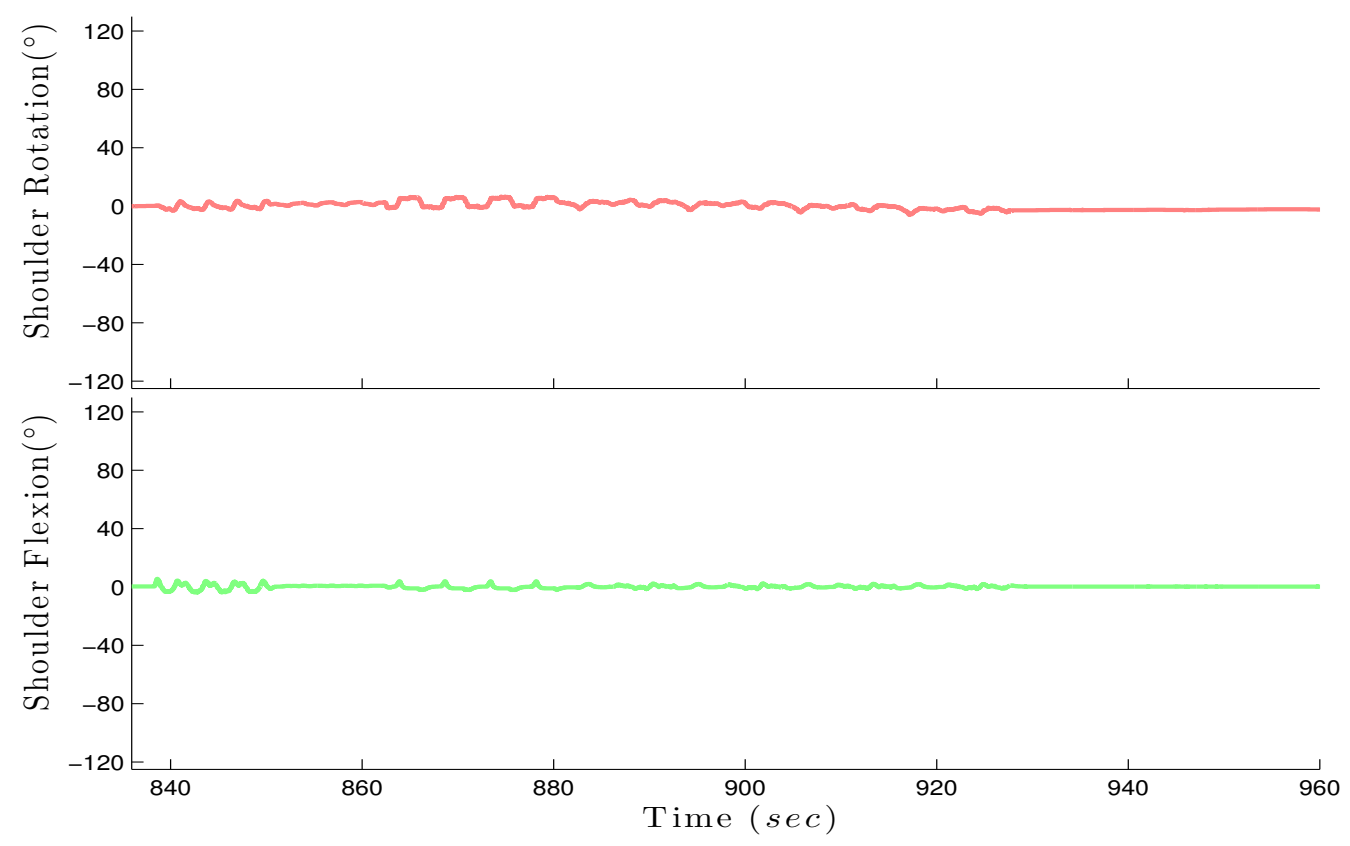

Figure 6.16: Shoulder angle error between true and estimates during the last 2 minutes. 


\subsection{Anatomical Constraints in Shoulder and Elbow}

In an attempt to circumvent the sensor drift problem and its effect on the accuracy of joint angle tracking, we use our knowledge of physical constraints and human natural range of motion to restrict angle estimates within acceptable range for different joints in the human body. Range of motion (ROM) refers to the amount of normal voluntary movement that a particular joint or body part can move, measured in degrees. Natural ROM is greatly determined by many factors such as age, gender, and bone structure. Researchers in medicine reported differences in joint range of motion in men and women. Studies have traditionally found females to have greater mobility and increased flexibility in all joints and angles except wrist and knee flexion [111]. Studies in sports reported increased ROM among athletes due to adaptive changes that lead to alterations in joint motion range [17]. On average, natural range of elbow flexion is between zero and $145^{\circ}$. The range of forearm supination is between zero and $85^{\circ}$, and between zero and $80^{\circ}$ for the forearm pronation. Wrist flexion/extension natural range is $\pm 75^{\circ}$. Human shoulder rotation around the humerus bone cannot exceed $90^{\circ}$. Similarly, shoulder cannot attain more than $180^{\circ}$ of abduction or flexion [42].

The state space framework provides a convenient means of incorporating prior knowledge of the dynamic system. There are many ways to incorporate state constraints into the nonlinear state estimators [109]. In this chapter, constraints are incorporated in the unscented Kalman filter algorithm during the time update step. The UKF uses a deterministic sampling technique known as the unscented transform to pick a set of sample points around the mean of the states. These points, known as sigma points, are then propagated through the nonlinear transition function to calculate the predicted states and their covariance. At this step, we restrict the predicted angle states within the natural range of motion region. The constrained predicted estimates are then used to update sigma points before they are propagated through the 
nonlinear measurement function to update the measurement estimates. During the measurement update, the constraints may be violated due to the linearization. However, these violations are rare and small in magnitude. The updated sigma points are also used to calculate Kalman gain, which in turn is used to calculate the state estimates. The result is a filter which more accurately captures the true states, and measurement mean and covariance.

\subsubsection{Results}

Table 6.6 shows a comparison between the true robot joint angles and their estimates using the inertial tracker with the modified kinematic equations. Results show that incorporating robot range of motion constraints greatly improved shoulder internal/external rotation angle estimates. The RMSE was dropped from $25.0^{\circ}$, obtained for baseline shoulder rotation, to $7.8^{\circ}$ for joint angle estimate with constraints. A perfect correlation and smaller errors were obtained between inertial estimates and true robot wrist, elbow, and forearm angles compared to the baseline results of the robot arm angles during slow rotation. Similar improvement was obtained for the other two data sets.

Table 6.6: RMSE of inertial angle estimates using algorithm with physical constraints compared to baseline results during slow rotation.

\begin{tabular}{lcr}
\hline \hline Task & $\operatorname{RMSE}\left(^{\circ}\right)$ & Baseline RMSE $\left(^{\circ}\right)$ \\
\hline Shoulder Internal/External Rotation & 7.8 & 25.0 \\
Shoulder Flexion/Extension & 0.8 & 1.1 \\
Elbow Flexion/Extension & 0.9 & 1.1 \\
Forearm Supination/Pronation & 1.3 & 1.4 \\
Wrist Flexion/Extension & 1.1 & 1.2 \\
Wrist Twist & 1.7 & 1.8 \\
\hline \hline
\end{tabular}




\subsection{Zero-Velocity Updates}

Another approach to reducing the effect of gyroscope drift on the estimated angles especially around the vertical axis during long movement periods is the use of zerovelocity updates (ZVU). This technique has been successfully used in a few ambulatory gait analysis and pedestrian navigation studies. During walking cycles, human feet alternate between a moving stride and a stationary stance phase when the foot is on the ground. In their tracking algorithm, Feliz et al. detected each stationary phase and used it to reset the angular rate to zero [40]. Resetting the inertial data to zero is refereed to as hard velocity update. Foxlin detected the stationary phase when gyroscope and accelerometer data stayed below a prescribed threshold for at least 0.15 seconds. He applied zero-velocity updates as pseudo-measurements in an EKF navigation error corrector [46]. This is classified as soft zero-velocity updates [110]. Zero-velocity updates is well suited and has been used for bounding the error growth of foot-mounted inertial sensors [108]. To the best of our knowledge, no study has used this approach in tracking joint angles or orientation of upper extremities.

In this chapter, we apply soft zero-velocity updates to update estimates of the gyroscope around the vertical axis. Since our algorithm uses gravity to estimate the attitude, and we only lack an absolute reference for heading about the vertical axis, we employ ZVU only around the vertical axis. When the rotational rate around the vertical axis stays below a threshold of $3 \%$ s for at least 0.25 seconds, movement is considered stationary and the measurement equation is augmented with a pseudo-measurement of gyroscope vertical axis random noise. Instead of applying hard update by resetting the velocity to zero, using pseudo-measurements into the UKF provides additional benefits. Besides the more accurate angle estimates around the vertical axis, the filter provides an estimate of the gyroscope bias and corrects rotational rate estimates. Thus, the filter corrects estimates of heading angle, and consequently other distal arm angles. 


\subsubsection{Results}

Table 6.7 shows a comparison between the true robot joint angles and their estimates using the inertial tracker with the modified kinematic equations. Results show that incorporating using zero-velocity update and the periodic correction improved shoulder internal/external rotation angle estimates. The RMSE was dropped from $25.0^{\circ} \mathrm{ob}-$ tained for baseline shoulder rotation to $12.2^{\circ}$ for the joint estimate employing ZVU. Negligible errors were obtained between inertial estimates and true robot wrist, elbow, and forearm angles compared to the baseline results of the robot arm angles during slow rotation. Similar improvement was obtained for the other two data sets.

Table 6.7: RMSE of inertial angle estimates using zero-velocity updates compared to baseline results during slow rotation.

\begin{tabular}{lrr}
\hline \hline Task & $\operatorname{RMSE}\left(^{\circ}\right)$ & Baseline RMSE $\left(^{\circ}\right)$ \\
\hline Shoulder Internal/External Rotation & 12.2 & 25.0 \\
Shoulder Flexion/Extension & 0.9 & 1.1 \\
Elbow Flexion/Extension & 1.0 & 1.1 \\
Forearm Supination/Pronation & 1.4 & 1.4 \\
Wrist Flexion/Extension & 1.4 & 1.2 \\
Wrist Twist & 1.8 & 1.8 \\
\hline \hline
\end{tabular}

\subsection{Modified Model with Bias, Constraints, and Zero-Velocity Updates}

We combine the three techniques discussed above into one modified arm model to account for sensor random drift, and to employ physical constraints and zero-velocity updates. The assessment of the modified inertial tracker performance in this section is based on the 15-minute recording of the arm rotating at medium rotational speed. Results will be compared to medium-speed baseline results discussed in Section 6.1.1. 


\subsubsection{Results}

Table 6.8 shows a comparison between the true robot joint angles and their estimates using the inertial tracker with the modified kinematic equations. Results show improved tracking and a reduced RMSE for all angles. The RMSE dropped from $8.1^{\circ}$ obtained for baseline shoulder internal/external rotation to $5.9^{\circ}$. A perfect correlation and smaller errors were obtained between inertial estimates and true robot elbow and shoulder flexion/extension angles. No apparent improvement to wrist angle tracking results. This is not surprising, since the three different approaches used to mitigate the effect of sensor random drift were applied to the measurement model of only the IMU placed on the upper arm. This unit measures only movement of the robot shoulder relative to the stationary robot base.

Table 6.8: RMSE of inertial angle estimates with modified arm model compared to baseline results during medium-speed arm rotation.

\begin{tabular}{lcc}
\hline \hline Task & $\mathrm{RMSE}\left({ }^{\circ}\right)$ & Baseline RMSE $\left(^{\circ}\right)$ \\
\hline Shoulder Internal/External Rotation & 5.9 & 8.1 \\
Shoulder Flexion/Extension & 2.1 & 2.4 \\
Elbow Flexion/Extension & 2.3 & 2.6 \\
Forearm Supination/Pronation & 2.1 & 2.1 \\
Wrist Flexion/Extension & 2.2 & 2.2 \\
Wrist Twist & 3.9 & 3.9 \\
\hline \hline
\end{tabular}

Fig. 6.17 and 6.18 show the last two minutes of the robot elbow and wrist movements at medium rotational rate. Shoulder angles during the first and last two minutes are shown in Fig. 6.19 and 6.20. Careful inspection of the results in all figures, especially shoulder results, shows an excellent agreement between true and inertial angle estimates. 


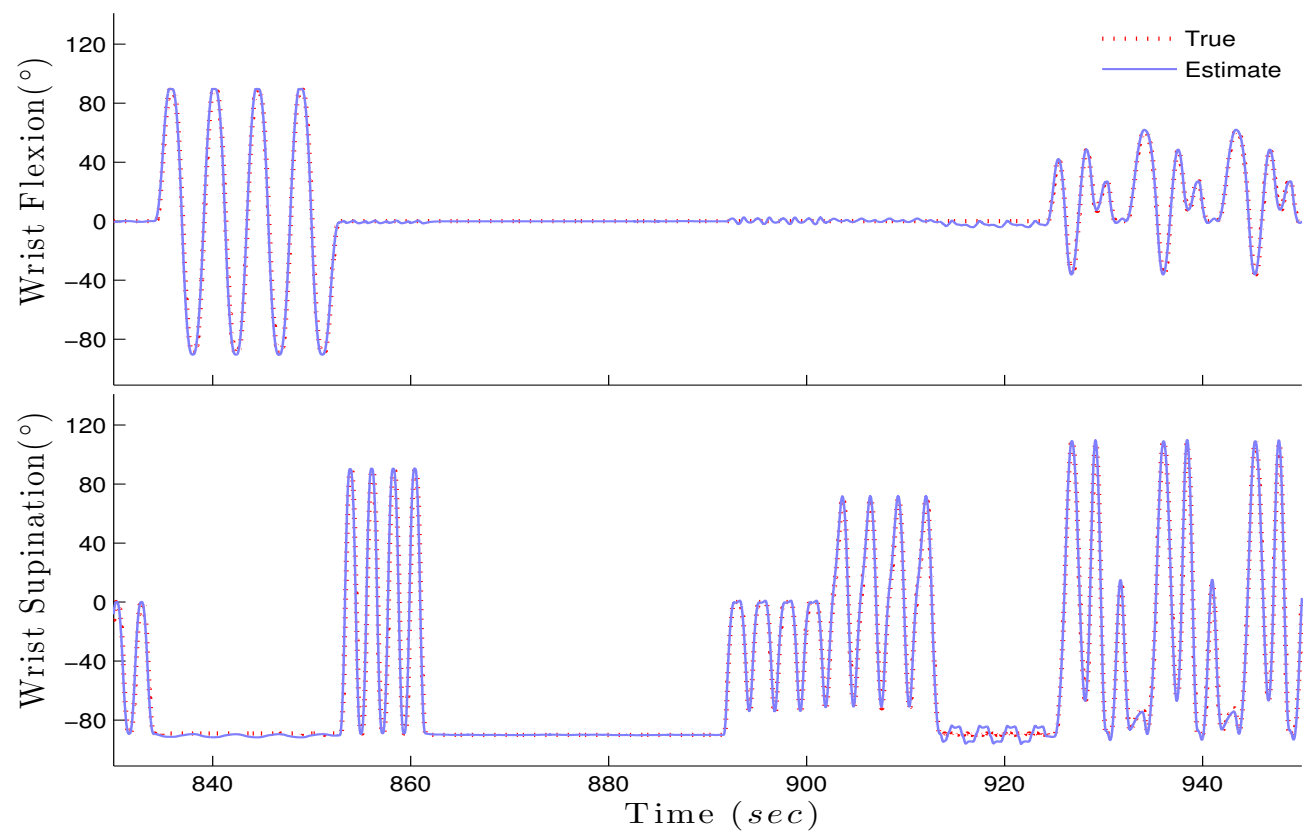

Figure 6.17: Wrist angles during the last 2 minutes at an average rotation rate of $318 \% \mathrm{~s}$.

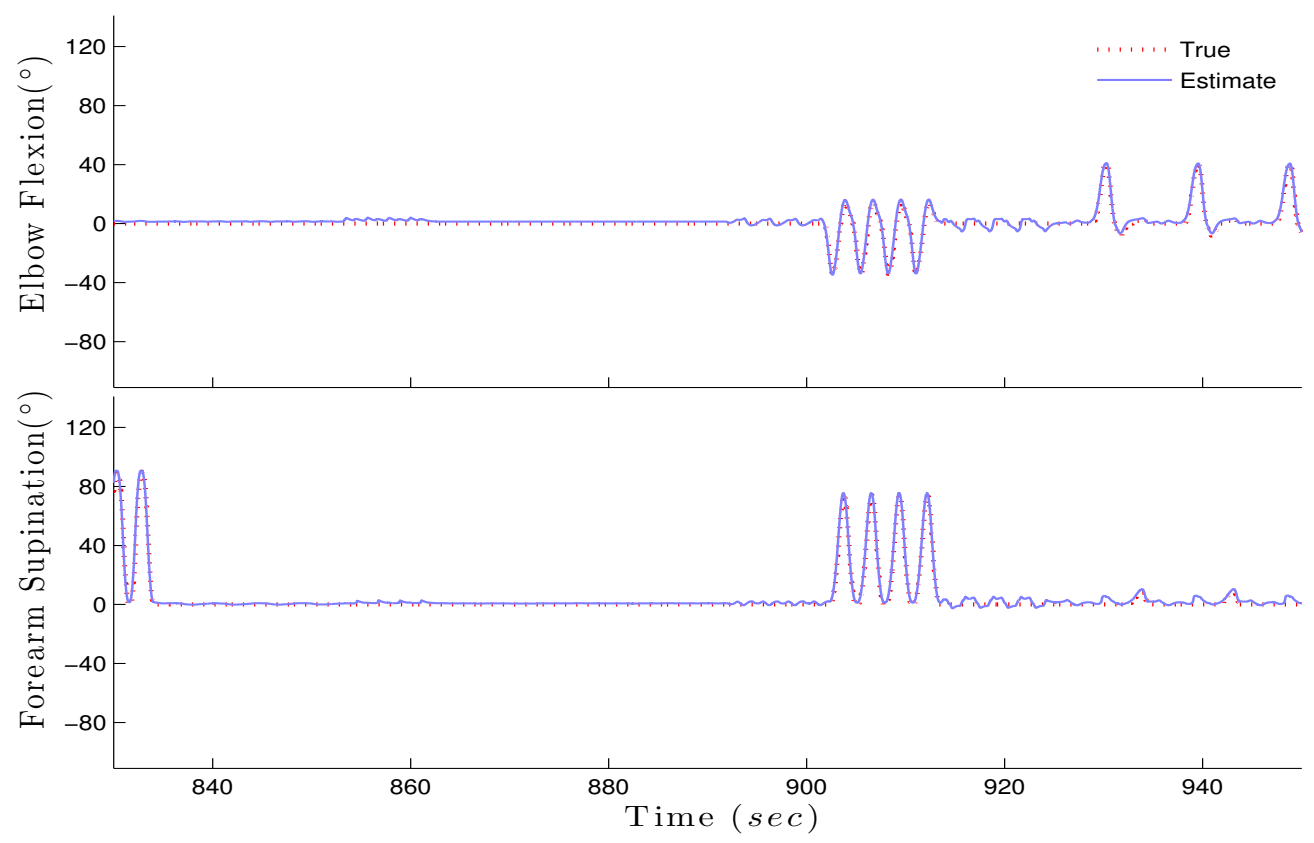

Figure 6.18: Elbow angles during the last 2 minutes at an average rotation rate of $266^{\circ} / \mathrm{s}$. 


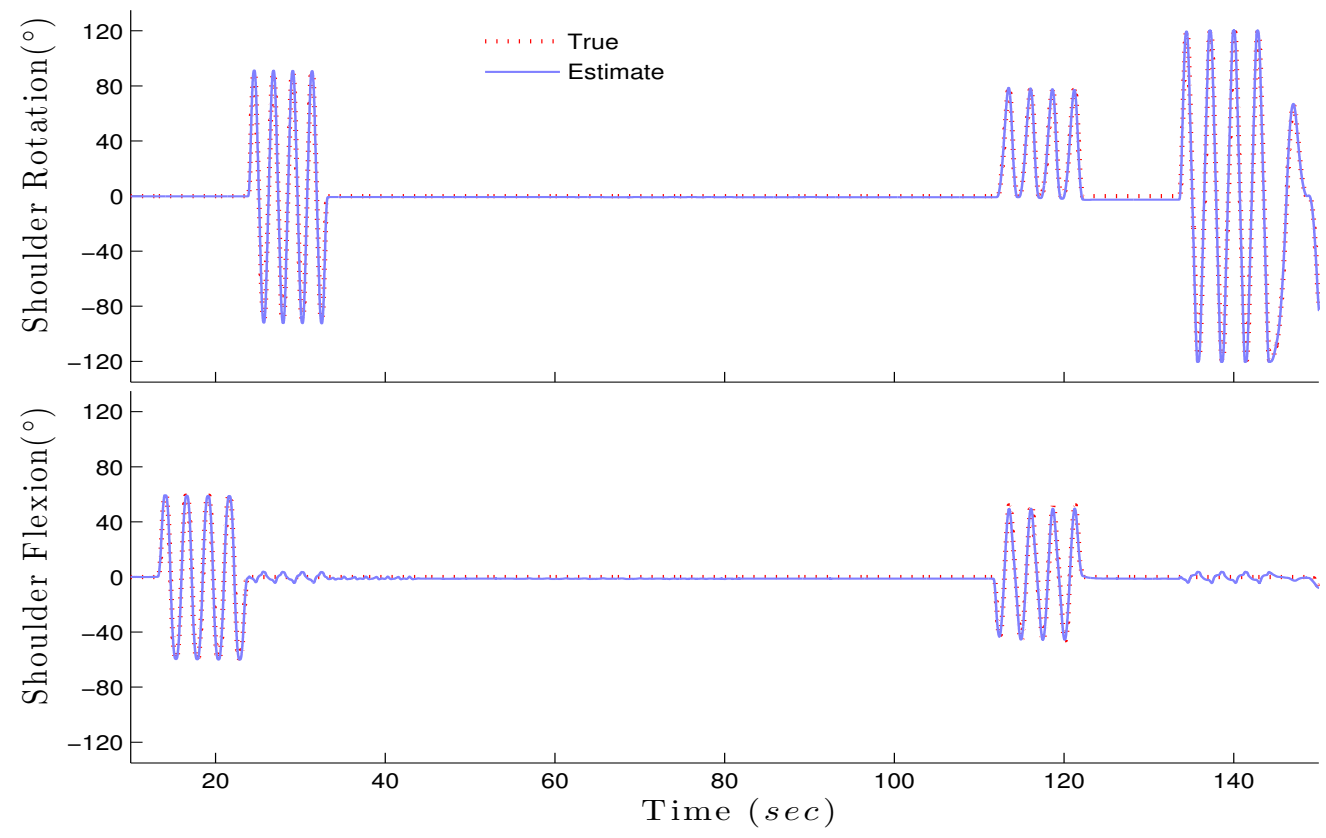

Figure 6.19: Shoulder angles of the first 2 minutes at an average rotation rate of $225^{\circ} / \mathrm{s}$.

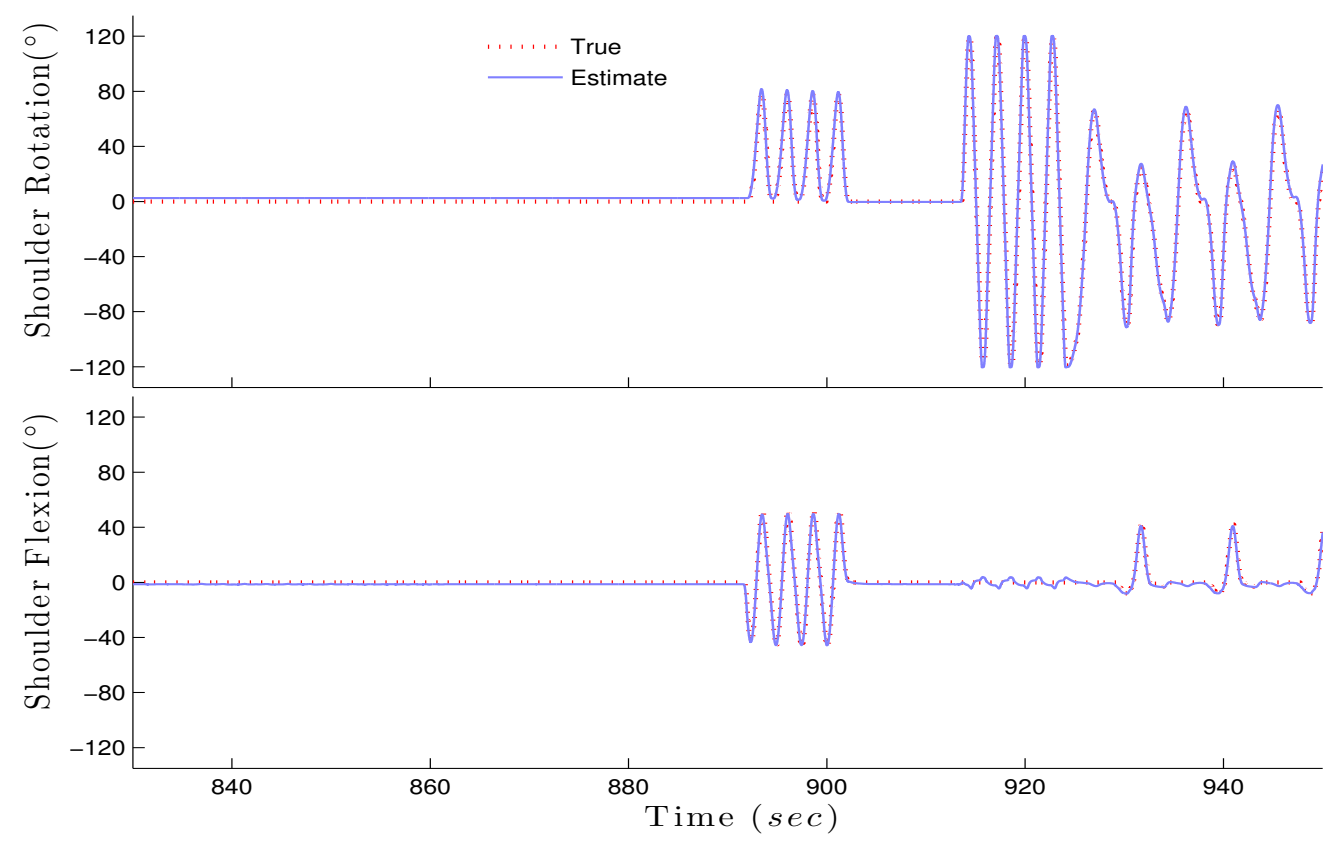

Figure 6.20: Shoulder angles of the last 2 minutes at an average rotation rate of $225^{\circ} / \mathrm{s}$. 


\subsection{Discussion}

Accelerometers and gyroscopes, as with most electronic sensors, are corrupted by random noise. Many research groups have used magnetometers to compensate the orientation error that occurs when integrating the angular rate. Fusion of magnetometers with inertial sensors offers enhanced performance only in the absence of magnetic field disturbances. In this chapter, we incorporated sensor random drift into the kinematic model, and used state space methods to employ zero-velocity updates and physical constraints to obtain better estimates of joint angles. The state space framework provides an elegant and efficient means of incorporating prior knowledge of the dynamic system. Careful inclusion of the prior knowledge leads to improved accuracy in tracking the system states.

We used the unscented Kalman filter with the modified system equations to estimate shoulder, elbow and wrist joint angles from an industrial robot arm with 6 degrees of freedom. Despite the different characteristics of human movement from that of a robot, we argue that using a robot arm for assessment has many advantages over the traditional optical systems. The different characteristics are mainly due to the type and DOFs of a joint. The shoulder joint is normally modeled as a ball-and-socket joint with 3 DOFs. However, the robot shoulder has only 2 DOFs, which limits the comparison to only 6 DOFs, including those of the elbow and wrist joints. Despite this limitation, using the robot arm for assessment provides many advantages. Unlike motion capture systems which require estimation of joint angles by processing marker positions and interpolation of missing markers data, the robot system provides direct angle measurements with high precision. The arm movement rate can be controlled to a desired rate ranging from slow to very fast, up to $720 \% \mathrm{~s}$. Furthermore, the robot provides a wide range of motion that can easily mimic human movement in performing various tasks.

Compared to true angles of the robot reference system, we achieved an average 
RMSE of less than $3^{\circ}$ during slow arm movement at a rotational rate $\leq 180^{\circ} / \mathrm{s}$. The modified process model with accelerometer and gyroscope sensors random bias resulted in a reduced maximum error of $5.2^{\circ}$ for heading angles around the vertical axis. This error is due to gyroscope drift accumulation after 15 minutes. This is a reasonable error range compared to what was reported by Roetenberg who showed that integration of noisy gyroscope data resulted in a drift between $10-25^{\circ}$ after one minute [96]. In contrast to many studies discussed in Chapter 2, we validated the performance of our tracking algorithm during different movement speeds over long durations of 15 minutes. Angle estimates during arm movement at medium rotation rate $\leq 360 \%$ s are very similar to those obtained during slow movement. On average, the RMSE was $3.3^{\circ}$, with a maximum error of $5.9^{\circ}$ between true and estimated internal/external shoulder rotation. The modified model resulted in a notable improvement to estimates around the vertical axis, compared to the baseline results shown in Fig. 6.10.

The error slightly increased during fast movement with an average RMSE of $3^{\circ}$, and a maximum error of $9.6^{\circ}$ between true and estimated shoulder internal/external rotation. Besides the effect of gyroscope drift on the accuracy of the estimated angles, there was another source of noise that contributed to the larger error. Due to the very fast arm movement, the table on which the arm is mounted was vibrating strongly, adding more noise to the sensor measurements. Despite the slightly higher tracking error during fast movement, we maintained a reasonable error range compared to what was achieved by other studies which reported an error range of $12^{\circ}-16^{\circ}$ [7].

The combined effect of imposing physical constraints on state estimates, modeling sensor bias, and zero-velocity updates can be observed especially during the last few minutes of the recording in Fig. 6.20. The effect of gyroscope drift on angle estimates greatly decreased to an acceptable error range. The RMSE was dropped from $25.0^{\circ}$ obtained with the baseline shoulder rotation to $7.8^{\circ}$ for estimates of the 
joint angles during slow rotation with an increased correlation from 0.92 to 0.99 . Similarly, the RMSE was dropped from $8.1^{\circ}$ with the baseline shoulder rotation to $5.9^{\circ}$ for estimates of the joint angles during medium-speed rotation. 


\section{Nonlinear State Estimation}

All relevant information about the state $x_{0: n}$ up to and including time $n$ can be obtained from the posterior density function $p\left(x_{0: n} \mid y_{0: n}\right)$. In Bayesian filtering, the state $x$ is assumed to be a hidden Markov process with an initial distribution $p\left(x_{0}\right)$. The goal is to recursively estimate the distribution $p\left(x_{n} \mid z_{0: n}\right)$, given all measurements $y_{1: n}$ up to time $n$. It is assumed that the prior $p\left(x_{0}\right)$ is known at time $n=0$ with no measurement $y_{0}$.

When the posterior density is Gaussian, the distribution is completely described by the state mean and covariance. When the state and observation models are linear and have Gaussian distribution, the recursive Kalman filter provides an optimal solution to the estimation problem. The solution is statistically optimal with respect to any quadratic function of estimation error. If the Gaussian assumption is relaxed, the Kalman filter is still a minimum variance estimator [94].

In practical situations, many systems of interest are nonlinear, and use of the linear Kalman filter may result in large estimation errors. A common approach to solving the nonlinear problem is to approximate the nonlinear functions, then apply the Kalman filter recursions to the linearized system. Approximation techniques for estimation of nonlinear systems include stochastic model approximation, function approximation and moment approximation [100]. In stochastic approximation, the nonlinear system is approximated by a simpler linear model. Function approximation has widely been implemented using Taylor series expansion. This is the basic idea of the extended Kalman filter (EKF), where the nonlinear functions are approximated 
by a first-order Taylor-series expansion. An example of the moment approximation technique is the unscented transformation, which is a central scheme in the unscented Kalman filter (UKF). The idea is to find a set of deterministic points in the state space that can adequately approximate the first and second moments (mean and covariance) of a distribution.

For non-Gaussian processes, the state mean and covariance are not sufficient to describe the distribution. Monte Carlo methods can be used to approximate the posterior density by a set of random samples, known as particles, with associated weights. Sequential Monte Carlo methods, also known as particle filters (PFs), carry out this approximation recursively to reduce the computational cost of the algorithm.

This chapter introduces a brief description of three nonlinear estimators: EKF, $\mathrm{UKF}$ and $\mathrm{PF}$, and evaluates their performance in tracking joint angles of the robot arm.

\subsection{Extended Kalman Filter}

The basic idea of the extended Kalman filter is to approximate the nonlinear process and measurement functions by a first-order Taylor-series expansion, then to apply the Kalman filter recursions during the update step . In other words, the Kalman filter and EKF have the same update equations.

The state and observations are governed by the following equations:

$$
\begin{aligned}
x(n+1) & =f_{n}[x(n), u(n)] \\
y(n) & =h_{n}[x(n), v(n)]
\end{aligned}
$$

where $x(n)$ represents the unobserved state of the system, and $y(n)$ represents the observed or measured data. The state and measurement noise are given by $u(n)$ and $v(n)$, respectively. They are assumed to be white noise with zero mean, and known 
covariance matrices $Q$ and $R$. The functions $f_{n}[\cdot]$ and $h_{n}[\cdot]$ are the nonlinear state and observation dynamics and are assumed to be known.

\subsubsection{Extended Kalman Filter Recursions}

Given a vector of noisy measurements $y_{n}$, estimation of the state $x_{n}$ is carried out in two steps similar to the Kalman filter algorithm. The first step is the prediction of $\hat{x}_{n \mid n-1}$ at time $n$ conditioned on all measurements through time $n-1$ :

$$
\begin{aligned}
& \hat{x}_{n \mid n}=f\left(\hat{x}_{n-1 \mid n-1}\right) \\
& P_{n \mid n}=F_{n} P_{n-1 \mid n-1} F_{n}^{\mathrm{T}}+Q_{n}
\end{aligned}
$$

where predicted states are propagated through the nonlinear function, whilst the predicted state error covariance $P_{n}$ propagates through the Jacobian $F_{n}$ :

$$
\begin{aligned}
& F_{n}=\left.\frac{\partial f_{n}(x)}{\partial x}\right|_{x=\hat{x}_{n \mid n}} \\
& H_{n}=\left.\frac{\partial h_{n}(x)}{\partial x}\right|_{x=\hat{x}_{n \mid n-1}}
\end{aligned}
$$

Given the predicted state estimate and its associated covariance obtained from past information, the filter calculates the Kalman gain $K_{n}$. This gain is used to obtain an updated and potentially better state estimate, when a new measurement of time $n$ becomes available.

$$
\begin{aligned}
K_{n} & =P_{n \mid n-1} H_{n}^{\mathrm{T}}\left(H_{n} P_{n \mid n-1} H_{n}^{\mathrm{T}}+R_{n}\right)^{-1} \\
\hat{y}_{n \mid n-1} & =h\left(\hat{x}_{n \mid n-1}\right) \\
\hat{x}_{n \mid n} & =\hat{x}_{n \mid n-1}+K_{n}\left(y_{n}-\hat{y}_{n \mid n-1}\right) \\
\hat{x}_{n+1 \mid n} & =f\left(\hat{x}_{n \mid n}\right) \\
P_{n+1 \mid n} & =F_{n} P_{n \mid n} F_{n}^{\mathrm{T}}+Q
\end{aligned}
$$

The state estimate covariance is also updated to reflect the new information, resulting in a reduced uncertainty. 


\subsection{Unscented Kalman Filter}

In the UKF, the state probability density is approximated by a deterministic sampling of points which represent the distribution as Gaussian. The underlying idea is to estimate the first and second order moments of the distribution from a set of $2 \ell+1$ points, called sigma points, with $\ell$ being the dimension of the state variable. Sigma points are generated deterministically assuming accurate knowledge of the mean and covariance matrix of the initial state.

\subsubsection{Unscented Kalman Filter Recursions}

Assuming we have an estimate of the mean $\hat{x}_{n}$ and the state error covariance $P_{n}$ at time $n$, we can find the sigma points as follows:

$$
\begin{array}{ll}
\chi_{n}^{i}=\hat{x} & \text { for } i=0 \\
\chi_{n}^{i}=\hat{x}+\sqrt{\ell_{x} P} & \text { for } i=1, \ldots, \ell_{x} \\
\chi_{n}^{i}=\hat{x}-\sqrt{\ell_{x} P} & \text { for } i=\ell_{x}+1, \ldots, 2 \ell_{x}
\end{array}
$$

The nonlinear functions are then applied to each of these sigma points as follows:

$$
\begin{aligned}
x_{n-1}^{i} & =\hat{x}_{n-1 \mid n-1}+\chi_{n-1}^{i} \\
\hat{x}_{n}^{i} & =f\left(\hat{x}_{n-1}^{i}\right) \\
\hat{x}_{n \mid n-1} & =\frac{1}{2 \ell_{x}} \sum_{i=1}^{2 \ell_{x}} \hat{x}_{n}^{i} \\
P_{\chi, n \mid n-1} & =\frac{1}{2 \ell_{x}} \sum_{i=1}^{2 \ell_{x}}\left(\hat{x}_{n}^{i}-\hat{x}_{n \mid n-1}\right)\left(\hat{x}_{n}^{i}-\hat{x}_{n \mid n-1}\right)^{\mathrm{T}}
\end{aligned}
$$


The measurement update is carried out as follows:

$$
\begin{aligned}
y_{n}^{i} & =h\left(x_{n}^{i}\right) \\
\hat{y}_{n \mid n-1} & =\frac{1}{2 \ell_{x}} \sum_{i=1}^{2 \ell_{x}} y_{n}^{i} \\
P_{y} & =\frac{1}{2 \ell_{x}} \sum_{i=1}^{2 \ell_{x}}\left(y_{n}^{i}-\hat{y}_{n \mid n-1}\right)\left(y_{n}^{i}-\hat{y}_{n \mid n-1}\right)^{\mathrm{T}}+R \\
P_{x y} & =\frac{1}{2 \ell_{x}} \sum_{i=1}^{2 \ell_{x}}\left(\hat{x}_{n}^{i}-\hat{x}_{n \mid n-1}\right)\left(y_{n}^{i}-\hat{y}_{n \mid n-1}\right)^{\mathrm{T}} \\
K_{n} & =P_{x y} P_{y}^{-1}
\end{aligned}
$$

And finally, the UKF uses the Kalman gain $K_{n}$ to calculate the state estimate and covariance:

$$
\begin{aligned}
\hat{x}_{n \mid n} & =\hat{x}_{n \mid n-1}^{i}+K_{n}\left(y_{n}-\hat{y}_{n \mid n-1}\right) \\
P_{\chi, n \mid n} & =P_{\chi, n \mid n-1}-K_{n} P_{y} K_{n}^{\mathrm{T}}
\end{aligned}
$$

It is argued that estimates of mean are accurate up to the third order when the distribution is Gaussian. However, this is based on the assumption that the mean and covariance of the initial state are known exactly. This is not true in practice, and that claim might not hold. Nonetheless, implementation of the UKF is substantially easier and requires no analytic derivation or Jacobian as in the EKF. However, the major drawback of the UKF is the need to compute a matrix square root that requires $\mathcal{O}\left(\ell_{x}^{3}\right)$ operations. 


\subsection{Particle Filters}

The key idea in particle filtering is to represent the posterior density function of the state by a set of random particles with associated weights, and to use these particles to compute the state estimates. At time $n$, the true posterior density can be approximated as a sum of weighted impulses:

$$
p\left(x_{0: n} \mid y_{0: n}\right) \approx \sum_{i=1}^{N_{\mathrm{p}}} w_{n}^{i} \delta\left(x_{0: n}-x_{0: n}^{i}\right)
$$

where $x_{o: n}^{i}$ represents the particles with their associated weights $w_{n}^{i}$, and $N_{\mathrm{p}}$ is the number of particles. Particles are randomly sampled from an importance density $q\left(x_{0: n} \mid y_{0: n}\right)$ using the principle of importance sampling [31]. The weights are updated as follows:

$$
w_{n}^{i}=\frac{p\left(y_{n} \mid x_{n}^{i}\right) p\left(x_{n}^{i} \mid x_{n-1}^{i}\right)}{q\left(x_{n}^{i} \mid x_{0: n-1}^{i}, y_{0: n}\right)} w_{n-1}^{i}
$$

where $p\left(y_{n} \mid x_{n}^{i}\right)$ is obtained from the measurement model, $p\left(x_{n}^{i} \mid x_{n-1}^{i}\right)$ from the process model, and $w\left(x_{n-1}^{i}\right)$ is obtained from the previous time step $n-1$. The weights are then normalized such that $\sum_{i=1}^{N_{\mathrm{p}}} w_{n}^{i}=1$.

Given that $q\left(x_{n}^{i} \mid x_{0: n-1}^{i}, y_{0: n}\right)=q\left(x_{n}^{i} \mid x_{n-1}^{i}, y_{n}\right)$, the importance density only depends on $x_{n-1}$ and $y_{n}$. Selection of the importance density is one of the filter design decisions, and it is one of the factors that control the tracking performance. The most common choice is the prior distribution,

$$
q\left(x_{n} \mid x_{0: n-1}, y_{0: n}\right)=p\left(x_{n} \mid x_{n-1}\right)
$$

However, this choice is sensitive to outliers and might lead to poor tracking. An optimal importance density is a function that results in particles contributing equally to the state estimates, with associated wights which have a minimum variance [3]. This optimal density is given by:

$$
q\left(x_{n} \mid x_{0: n-1}, y_{0: n}\right)=p\left(x_{n} \mid x_{n-1}^{i}, y_{n}\right)
$$


However, this optimal density requires the ability to draw from $p\left(x_{n} \mid x_{n-1}^{i}, y_{n}\right)$, which can not be done. A suboptimal approach is to approximation the optimal density with a Gaussian distribution. The importance weight are calculated recursively, and then normalized as follows:

$$
\begin{aligned}
\tilde{w}_{n}^{i} & =p\left(y_{n} \mid x_{n}^{i}\right) w_{n-1}^{i} \\
w_{n}^{i} & =\frac{1}{\sum_{i=1}^{N_{\mathrm{p}}} \tilde{w}_{n}^{i}} w_{n-1}^{i}
\end{aligned}
$$

Computational cost of particle filters can be adjusted by increasing or decreasing the number of particles. This, in turn, controls the tradeoff between accuracy of state estimates and computation. The computation required at each time step is $\mathcal{O}\left(N_{\mathrm{p}}\right)$.

One of the drawbacks of the particle filters is known as the degeneracy problem, when after a few iterations, all but a few of the particles have negligible weight. This implies that a large computational effort is spent updating particles that have no contribution to the state estimates. To avoid degeneracy, filters utilize a resampling routine to replicate particles with large importance weights and eliminate the ones with negligible weights. Although resampling increases the computation complexity of the filter, it leads to better state estimates. Resampling is performed when the variance of the weights reaches a pre-specified threshold. In other words, when the effective sample size falls below the threshold. The effective sample size is defined as:

$$
N_{\mathrm{pe}}=\left(\sum_{i=1}^{N_{\mathrm{p}}} w_{n}^{i}\right)^{-1}
$$

The particle filter results presented in the following section were generated with 500 particles, and resampling was performed when the effective number fell below 125 particles. 


\subsection{Results}

Performance and comparison of the EKF, UKF and PF are based on a $30 \mathrm{~s}$ segment of the robot arm data introduced in Chapter 6. Three inertial measurement units, each containing triaxial accelerometers and gyroscopes, were placed on the upper arm, forearm and wrist of the robot as shown in Fig. 6.1. A list of the user-specified filter parameters used to generate the results introduced in this section is shown in Table 7.1.

Table 7.1: User-specified parameters and sample rate used with the three filter. $I$ represents an identity matrix.

\begin{tabular}{lcr}
\hline \hline Name & Symbol & Value \\
\hline Variance of gyroscope measurement noise & $\sigma_{v_{g}}{ }^{2}$ & .0001 \\
Variance of accelerometer measurement noise & $\sigma_{v_{a}}{ }^{2}$ & .01 \\
Variance of process noise & $\sigma_{u}{ }^{2}$ & 10 \\
Initial state covariance matrix & $P$ & $I$ \\
Angular acceleration process parameter & $\alpha$ & .95 \\
Sample rate & $f_{s}$ & $128 \mathrm{~Hz}$ \\
\hline \hline
\end{tabular}

True robot shoulder internal/external rotation and flexion/extension angles (dashed red lines) and their estimated values (solid blue lines) using the UKF are shown in Fig. 7.1. Similarly, true angles of the elbow, forearm and wrist, and their estimates are shown in Fig. 7.3 and 7.5.

Fig. 7.2 shows the error between true shoulder rotation and estimates from the three filters. Estimation errors are less than $3^{\circ}$ for both of the shoulder internal/external rotation and flexion/extension angles. Unlike the EKF and PF, the UKF error is consistently negligible and stays almost constant throughout the shoulder flexion/extension segment.

The error between true elbow and forearm angles, and their estimates are shown in Fig. 7.4. Estimates by the UKF are almost identical to the true elbow and forearm angles, and the estimation error is consistently low. Comparable performance of 


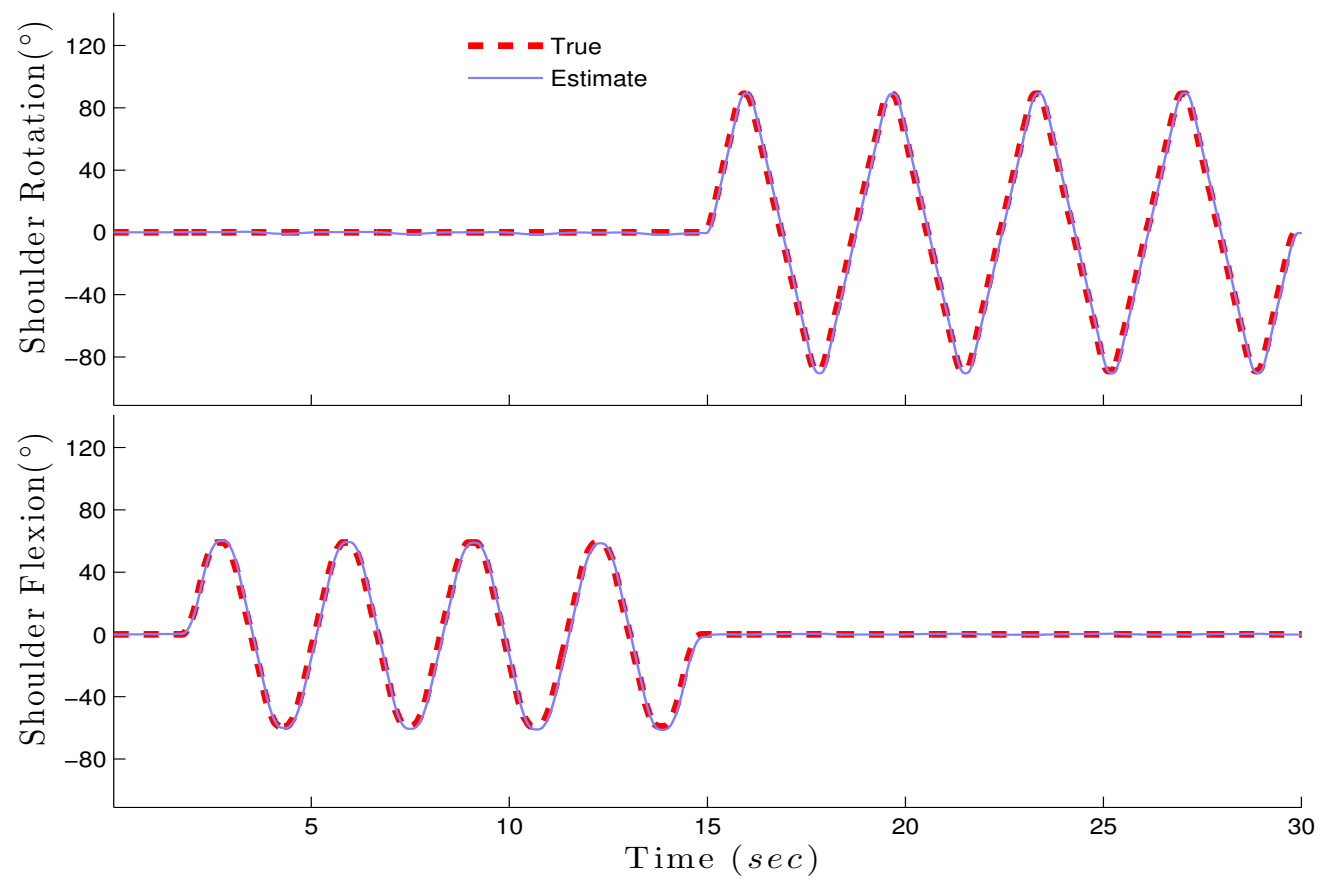

Figure 7.1: True robot shoulder rotation and flexion, and their estimates using UKF.

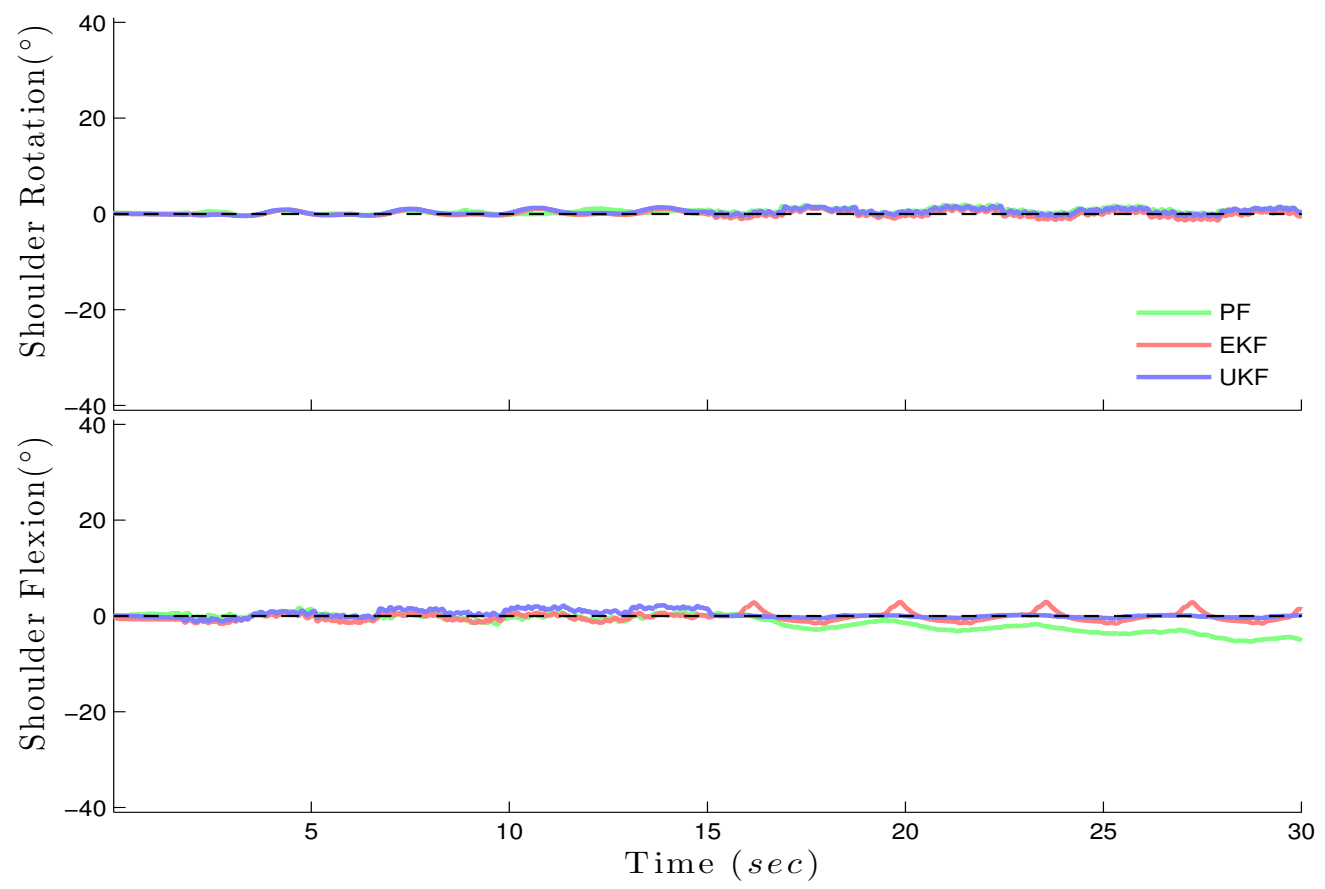

Figure 7.2: Error between true robot shoulder angles and estimates using EKF, UKF and PF. 


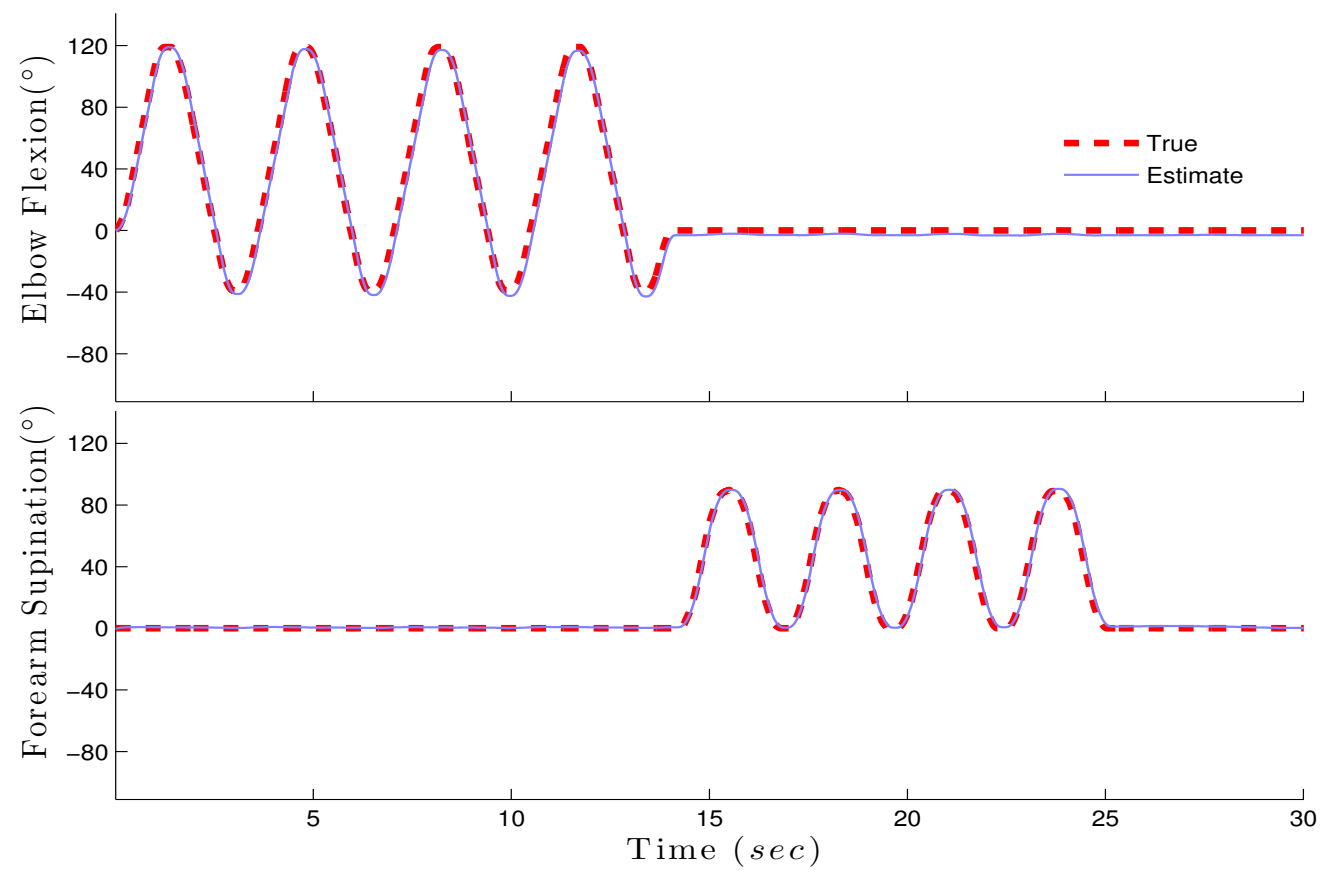

Figure 7.3: True robot elbow flexion and supination, and their estimates using UKF.

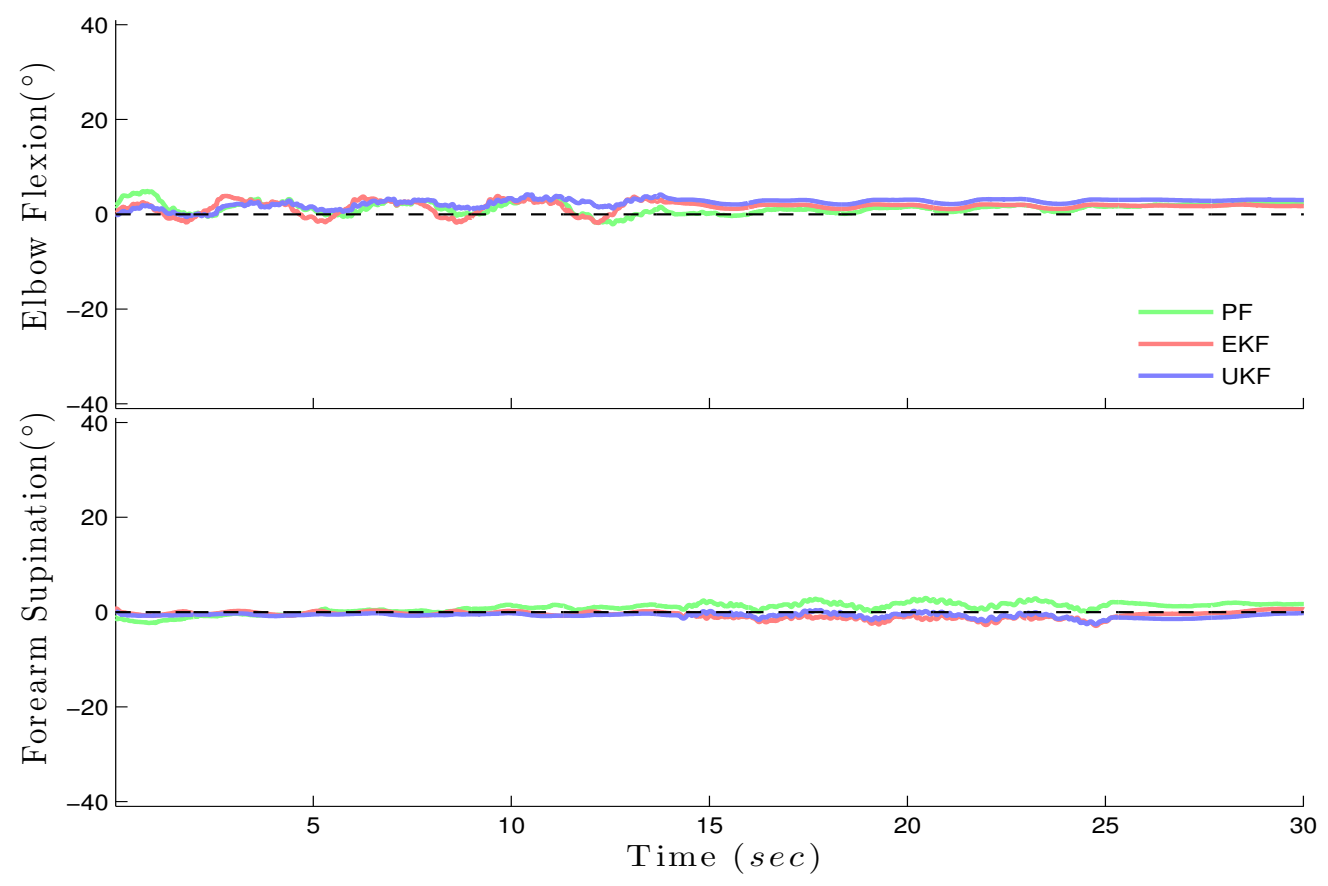

Figure 7.4: Error between true robot elbow angles and estimates using EKF, UKF and PF. 


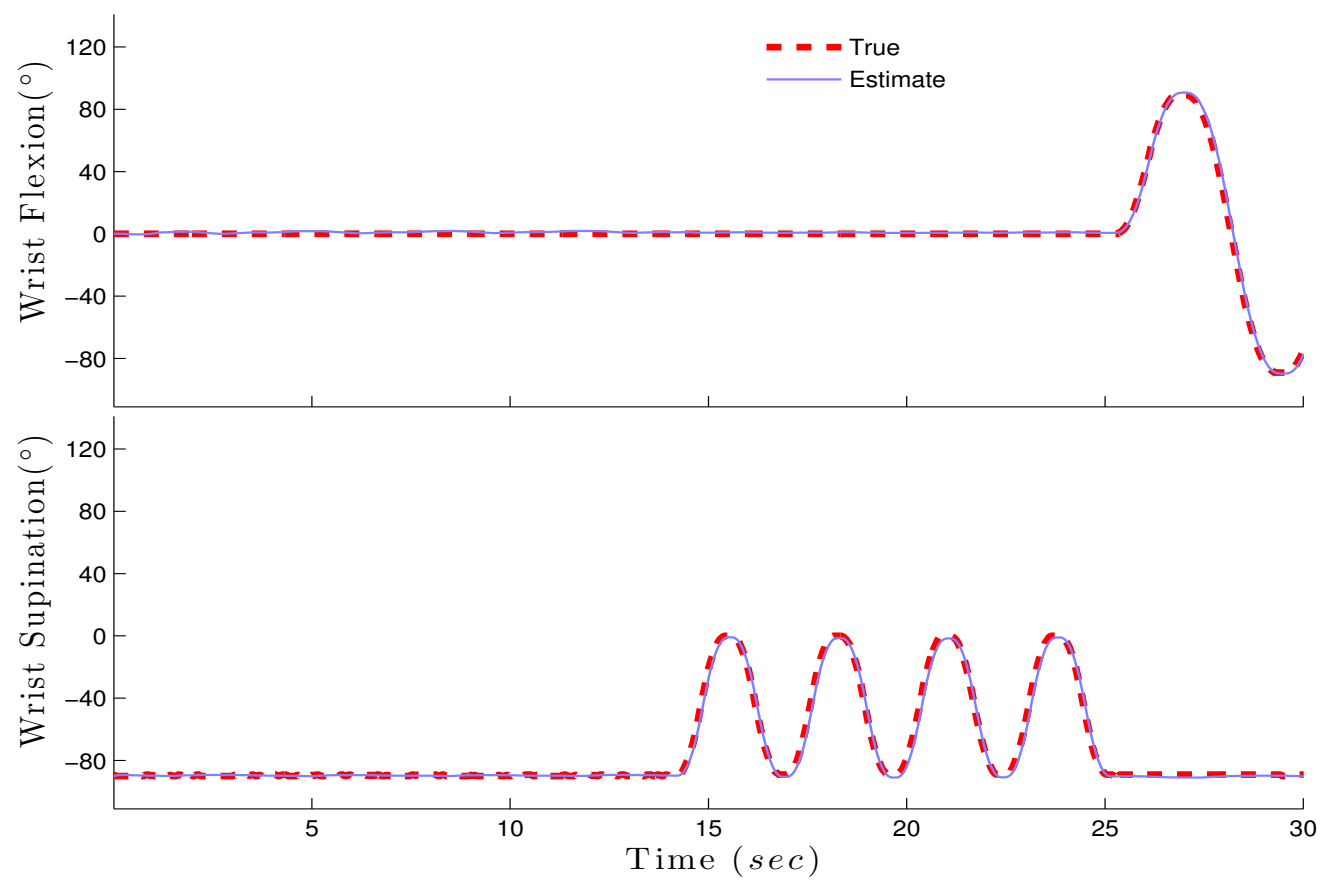

Figure 7.5: True robot wrist flexion and supination, and their estimates using UKF.

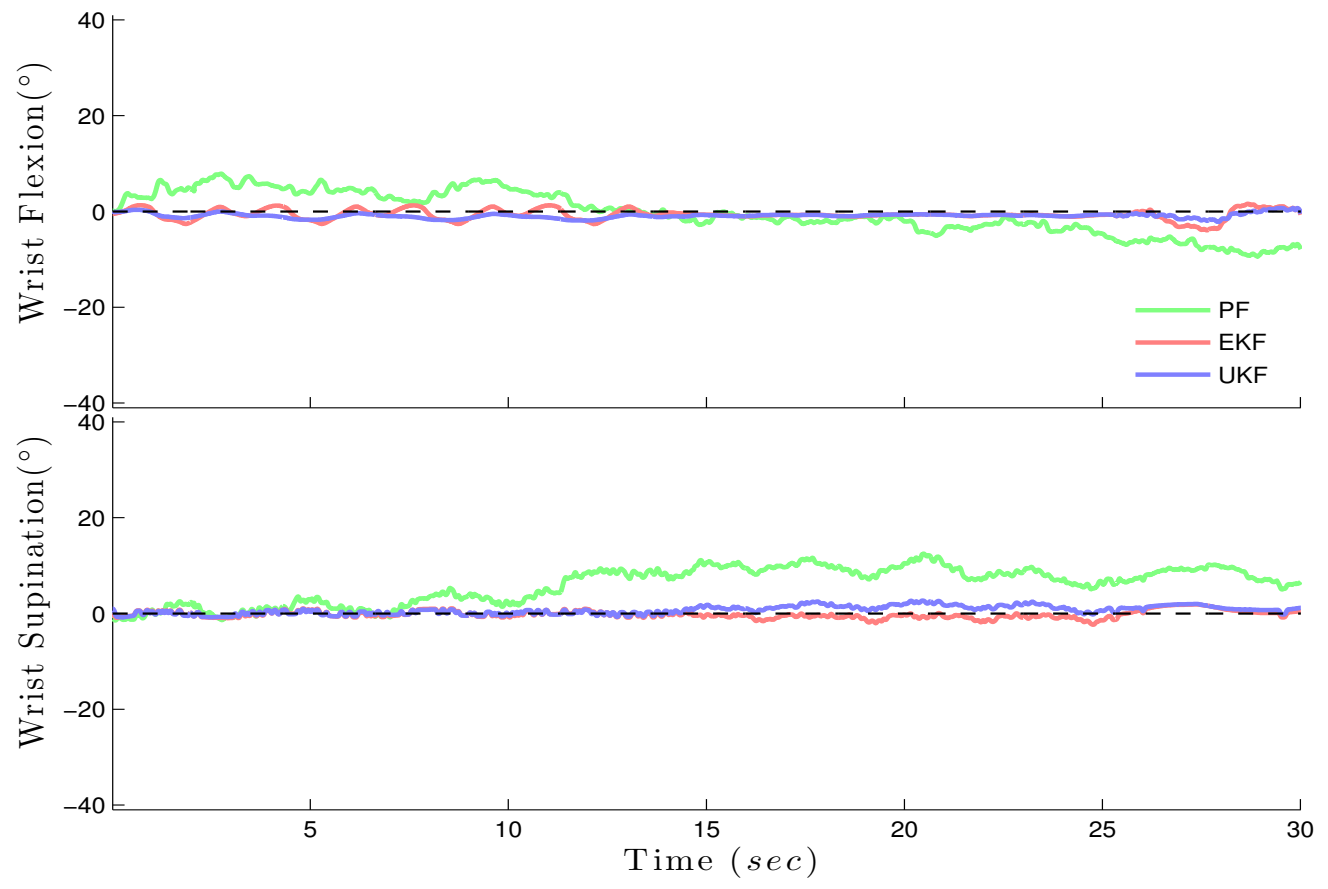

Figure 7.6: Error between true robot wrist angles and estimates using EKF, UKF and PF. 
the PF and EKF in tracking elbow and forearm angles can also be seen in Fig. 7.4. On the other hand, the PF estimation error of the wrist angles increased with time. Fig. 7.6 shows that the PF initially wanders for a few seconds until it locks on the wrist flexion state. The filter looses track of the angle states a few seconds later.

Increasing the number of particles from 500 to 2500 resulted in improving the PF tracking. Table 7.2 shows the RMSE between the true robot joint angles and their estimates using the EKF, UKF, and PF with $500\left(\mathrm{PF}_{500}\right)$ and $2500\left(\mathrm{PF}_{2500}\right)$ particles.

The number of floating-point operations (flops) employed in an algorithm is normally used to analyze the computational complexity of the algorithm. A flop is defined as on of addition, subtraction, multiplication, or division of two floating-point numbers. However, to compare the computational complexity by giving the number of flops employed in each of the three filters, we run into a few problems. There are certain steps in the filters algorithm that cannot be measured using flops, including the cost of generating a random number and the cost of evaluating a certain nonlinear function. Nonetheless, it is still possible to analyze the complexity by measuring the absolute time required to execute the different filters. Along with the average error among all angles, Table 7.2 compares the filters computational requirement time in seconds.

Table 7.2: Root mean squared error (RMSE) in degrees between true robot arm angles and estimates using the EKF, UKF and PF with 500 and 2500 particles.

\begin{tabular}{lcccr}
\hline \hline Task & $\mathrm{EKF}$ & $\mathrm{UKF}$ & $\mathrm{PF}_{500}$ & $\mathrm{PF}_{2500}$ \\
\hline Shoulder Internal/External Rotation & 2.9 & 2.9 & 2.4 & 0.7 \\
Shoulder Flexion/Extension & 2.3 & 2.1 & 2.9 & 1.5 \\
Elbow Flexion/Extension & 3.7 & 2.9 & 3.2 & 2.6 \\
Forearm Supination/Pronation & 2.2 & 1.7 & 2.3 & 1.3 \\
Wrist Flexion/Extension & 1.8 & 1.4 & 3.4 & 1.3 \\
Wrist Twist & 2.2 & 1.7 & 7.8 & 2.3 \\
\hline Average error $\left(^{\circ}\right)$ & 2.5 & 2.1 & 3.7 & 1.6 \\
Execution time (s) & 38 & 66 & 670 & 3430 \\
\hline \hline
\end{tabular}




\subsection{Discussion}

Rather than approximating the nonlinear functions like the EKF, the UKF approximates the probability distribution. Compared to the tracking performance by the EKF, the UKF resulted in better estimates and a reduced error in five of the six robot angles. The average error reduction was about $16 \%$ of the EKF average error. The UKF performance improvement was achieved at the expense of an increased computational requirement. The UKF recursions were executed in almost twice the time of the EKF. Performance of the EKF and UKF is dependent on the choice of initial state estimate and other parameters listened in Table 7.1. The same parameters were used with the three filters. Unlike the EKF, the UKF does not require the evaluation of the Jacobians of the functions $f_{n}[\cdot]$ and $h_{n}[\cdot]$. Hence, the UKF represents a better alternative to the EKF whenever a linearized model is not accurate enough or the Jacobian computation becomes too cumbersome.

Compared to the particle filter with 500 particles, the UKF resulted in better estimates and a reduced error in five of the six arm angles. On average, the RMSE of the PF was $3.7^{\circ}$ compared to $2.1^{\circ}$ and $2.5^{\circ}$ of the UKF and EKF, respectively. The UKF average error was approximately $43 \%$ of the PF estimation error, and its recursions were executed in less than one tenth of the execution time of the PF.

While the UKF estimates the mean and covariance of the state, the PF estimates the PDF as the number of particles approaches infinity [24]. Given enough particles, a particle filter performs better than a UKF, but this might be at the expense of unacceptable computational requirements. Increasing the number of particles and the frequency of resampling improved tracking. The average RMSE dropped from $3.7^{\circ}$ to $1.6^{\circ}$, an error reduction of $57 \%$. However, large number of particles and repeated resampling increases the computational requirements of the filter. Moreover, with repeated resampling, particles loose diversity, and tracking fails with only a few particles contributing to the state estimate. This problem is known as sample impover- 
ishment.

Various algorithms have recently been developed to improve the performance of particle filters and to address sample degeneracy and the impoverishment problem. Efforts have been devoted to selecting an appropriate importance density and to modification of the resampling process [50, 90, 86]. Cappè et al. provide an overview of existing methods and recent advances in particle filters [19]. Utilizing one of the modified particle filters results in improved performance in tracking joint angles. However, particle filters are still limited by the computational requirements proportional to the number of particles needed for reasonable tracking results. The number of particles scales with the state dimension $\ell$. This proves to be problematic, especially in human movement tracking applications. The state dimensions grow proportionally with the number of angles included in the kinematic model of the body, and the filter performance often deteriorates with the increased state dimensions. 


\section{Summary And Conclusion}

The objective of the research presented in this dissertation is to overcome the technological and methodological difficulties and limitations associated with existing human joint tracking techniques. We developed an algorithm that utilizes wireless wearable inertial sensors to directly and continuously estimate human joint angles. Tracking is performed in real-time or off-line with high accuracy for slow, normal and fast, as well as complex movement with a minimal number of inertial sensors. The algorithm combines kinematic models designed for control of robots with state space methods to accurately estimate human joint angles.

\subsection{Summary}

The need to characterize normal and pathological human movement has consistently driven researchers to develop new and improved tracking systems. Interest in tracking human movement has been motivated by its wide array of applications including: diagnosis of neurological movement disorders and assessment of treatment, rehabilitation from motor system injuries, as well as analysis of athletes' performance in professional sports, to name a few.

Human movement has traditionally been captured by either optical, mechanical, magnetic, acoustic, or inertial systems. Although each of these systems has its own advantages, they all suffer from various limitations and shortcomings. Optical systems are costly, require fixed cameras in a controlled environment, and suffer from 
problems of occlusion, similar to those in acoustic and structured light systems. Magnetic and radio frequency systems suffer from electromagnetic disturbances, noise and multipath problems. Mechanical systems have physical constraints that limit the natural body movement. These systems and their limitations were discused in Chapter 1.

Inertial measurement units (IMUs), on the other hand, offer a practical and relatively low-cost alternative to other motion capture systems. Wireless IMUs can be attached to various body segments, and can be used in and outside of a laboratory. Movement can continuously be recorded while subjects perform normal daily activities over an extended period, and data can be processed off-line or during real-time. In Chapter 2, we reviewed some of the traditional and recently developed inertial tracking algorithms and their limitations. In these algorithms, orientation estimates are accurate for brief and static periods when accelerometers could be used as inclinometers. Studies using magnetometers reported large orientation errors when sensors were in the vicinity of magnetic field disturbances. Another factor limiting the success of some of these tracking algorithms is the number of measurement units required to track complex human movement. Large numbers of sensors increase the cost and the computational complexity of the system.

Contrary to previous studies, the research and performance evaluation presented in this dissertation demonstrates the feasibility of tracking human joint angles with a minimal configuration of IMUs including only triaxial gyroscopes and accelerometers. As discussed in Chapter 4, joint angles are estimated using a new tracking algorithm that does not require magnetic sensors. In addition to eliminating the error due to magnetic disturbances, this reduces the size of process and measurement models, and consequently reduces the computational requirement of the algorithm. The inertial tracker utilizes both gravity and translational acceleration, together with rotational rate, making it suitable for tracking slow and fast movement with excellent 
accuracy. An integral part of the inertial tracker is a novel measurement model. The model is based on Newton-Euler dynamics utilized in the control of robotic arms introduced in Chapter 3.

To evaluate the system and tracking performance, we compared joint angles estimated by the inertial tracker to those estimated by an optical tracking reference system. Shoulder and elbow movement data from 8 subjects were used to evaluate the performance. Tracking results were evaluated in Chapter 5. On average, the RMSE was less than 8 degrees for all shoulder and elbow angles. The average correlation coefficient for all movement tasks among subjects was $r \geq 0.95$. This agreement between our inertial tracker and the optical reference system was obtained for both regular and high-speed arm movement.

A major source of error in tracking human kinematics using inertial sensors is inherent sensor bias and random drift. When gyroscope data is integrated, this drift introduces an angle error that grows proportionally with time. In Chapter 6, we introduced a modified process and measurement model to reduce the effect of sensor drift and to increase the tracking accuracy. The modified tracker incorporates gyroscope and accelerometer random drift models, utilizes physical constraints and natural range of human motion, and applies zero-velocity updates to provide periodic correction to the error in angle estimates. Joint angles calculated by the modified inertial tracker were compared to those obtained from a high precision industrial robot arm with six degrees of freedom, during 15-minute recordings for slow, regular and high-speed movement. The combined effect of imposing physical constraints, modeling sensor bias, and employing zero-velocity updates resulted in a considerable decrease in tracking error. The RMSE dropped from $25.0^{\circ}$ obtained with the baseline heading angle to $7.8^{\circ}$ for estimates during slow rotation, an error reduction of $69 \%$. Similarly, the RMSE dropped from $8.1^{\circ}$ with the baseline heading angle to $5.9^{\circ}$, an estimation error reduction of $27 \%$ for joint angles during medium-speed rotation. 
Using the robot arm for assessment provides many advantages. Unlike motion capture systems, the robot system provides direct angle measurements with high precision. The arm movement rate can be controlled to a desired speed ranging from slow to very fast, up to $720 \%$ s. Furthermore, the robot provides a wide range of motion that can easily mimic human movement in performing various tasks.

The tracker utilizes the unscented Kalman filter (UKF), which is an improvement to the extended Kalman filter (EKF). The UKF removes the need for linearization while providing estimates that capture the statistics of the target distribution more accurately than the EKF [70]. The UKF approximates the distribution rather than the nonlinearity, therefore it is accurate to at least the second order. Moreover, the UKF eliminates the need for Jacobian matrices.

Particle filters (PFs), provide an alternative to the EKF and UKF in solving nonlinear and non-Gaussian estimation problems. In Chapter 7, we introduced a brief description of the EKF, UK and PF, and evaluated their performance in tracking the robot arm angles. Compared to the EKF, the UKF resulted in better estimates and a slightly reduced error in five of the six robot arm angles. The average error reduction was approximately $16 \%$ of the EKF error. UKF performance improvement was achieved at the expense of a little increase in execution time of the UKF recursions. Similarly, the UKF resulted in a better joint angle tracking than the PF. The UKF average error reduction was approximately $43 \%$ of the PF error. Furthermore, the efficient UKF recursions required less than one tenth of the PF execution time. Given enough particles, a particle filter performs better than a UKF, but this might be at the expense of unacceptable computational requirements.

\subsection{Contributions}

The key contribution of the research presented in this dissertation is the development of an inertial joint angle tracker which overcomes the limitations of existing 
human joint tracking systems. The inertial tracker integrates a biomechanical model of human limbs with state space methods to solve the kinematic problem. That is, determining human joint angles given angular velocity and acceleration measurements. The algorithm can be applied to any combination of inertial sensors, and can efficiently be generalized to track movement of any combination of human limbs. The implementation can use tracking algorithms that are either causal, real-time or non-causal, offline smoothing with higher accuracy. Here are the contributions made in this dissertation:

- Expression of a biomechanical model and integrating it with state space methods to track human movement. The inertial tracker combines a novel process and measurement model with state space methods to track human joint angles using wearable inertial sensors. The biomechanical model greatly reduces the complexity of the tracking problem, resulting in an efficient tracking algorithm that is capable of tracking complex human movement with great accuracy.

- Generalization of the state space model to account for sensor noise and drift. The state space framework provides an elegant and efficient means of incorporating gyroscope and accelerometer random drift models into the process and measurement equations to reduce the effect of sensor drift, and to increase the tracking accuracy.

- Integration of biomechanical limits and prior knowledge of arm movement constraints into the kinematic model and the tracking algorithm. To circumvent the sensor drift problem and its effect on the accuracy of joint angle tracking, we use prior knowledge of physical constraints and human natural range of motion to restrict angle estimates within acceptable range for different joints in the human body. 
- Using Zero-Velocity Updates to provide periodic correction to the error in angle estimates. This is another approach to reducing the effect of gyroscope drift on estimated angles, especially around the vertical axis during long movement periods. This technique has been successfully used in a few ambulatory gait analysis and pedestrian navigation studies. To the best of our knowledge, no study has used this approach in tracking joint angles or the orientation of upper extremities. Rather than applying hard updates by resetting the velocity to zero, we augment the measurement equations with a pseudo-measurements vector. Besides the improved accuracy of angle estimates around the vertical axis, the augmented vector provides an estimate of the gyroscope bias and corrects rotational rate estimates. Therefore, the filter corrects estimates of heading angle, and consequently other distal arm angles.

- Validation of the inertial tracking algorithm using two reliable reference systems. Joint angles obtained from the inertial tracker were compared to those obtained from an optical tracking system and a high-precision industrial robot arm. Optical systems are the most common and the most accurate in tracking human movement. The robot system provides direct angle measurements with high precision. The arm movement rate can be controlled to any desired speed, up to $720^{\circ} / \mathrm{s}$ to assess the inertial algorithm in tracking slow, normal and fast, as well as complex movement.

- Developing the inertial tracker with three different nonlinear estimators to determine the most efficient tracking algorithm, and quantifying the tradeoff between the performance and computational requirements of the UKF, EKF and PF. 


\subsection{Conclusion}

The results presented in this dissertation demonstrate that wearable inertial sensors have the potential to achieve a level of accuracy that facilitates the study of normal and pathological human movement. We combined kinematic models designed for control of robotic arms with state space methods to directly and continuously estimate human joint angles using wearable inertial sensors. These algorithms can be applied to any combination of inertial sensors, and can be generalized to track any limb movement. The state space framework enables one to efficiently model sensor imperfections, to impose physical constraints on state estimates, and to track movement in real-time or with improved accuracy offline. The agreement with an optical motion system and a high-precision robot arm reference system was excellent. Unlike other motion systems, which require fixed cameras in a controlled environment and suffer from problems of occlusion, wearable inertial sensors can be used anywhere, cannot be occluded, and are low cost. Our proposed method uses a minimal sensor configuration with one IMU on each segment. In addition, our method is very accurate during long periods of movements at various rotational rates. 


\section{References}

[1] Hussein A. Abdullah, Cole Tarry, Rahul Datta, Gauri Mittal, and Mohamed Abderrahim. Dynamic biomechanical model for assessing and monitoring robot-assisted upper-limb therapy. Journal of Rehabilitation Research and Development, 44(1):43-62, November 2007. [cited at p. 4]

[2] Kamiar Aminian, B. Najafi, C. Büla, P-F. Leyvraz, and P. Robert. Spatiotemporal parameters of gait measured by an ambulatory system using miniature gyroscopes. Journal of Biomechanics, 35(5):689-699, May 2002. [cited at p. 15]

[3] Sanjeev Arulampalam, Simon Maskell, Neil Gordon, and Tim Clapp. A Tutorial on Particle Filters for Online Nonlinear/Non-Gaussian Bayesian Tracking. IEEE Transactions on Signal Processing, 50(2):174-189, February 2002. [cited at p. 92]

[4] Ronald Azuma and Gary Bishop. Improving Static and Dynamic Registration in an Optical See-through HMD. In 21st Annual Conference on Computer Graphics, pages 197-204, 1994. [cited at p. 5]

[5] Eric R. Bachmann and Robert B. McGhee. Inertial and magnetic posture tracking for inserting humans into networked virtual environments. In $A C M$ Symposium on Virtual Reality Software and Technology, pages 9-16. ACM: New York, NY, 2001. [cited at p. 16] 
REFERENCES

[6] Eric R. Bachmann, Xiaoping. Yun, and Ann Brumfield. Limitation of Attitude Estimation Algorithms for Inertial/Magnetic Sensors Modules. Robotics \& Automation Magazine, September:76-87, September 2007. [cited at p. 16]

[7] Eric R. Bachmann, Xiaoping Yun, and Christopher Peterson. An investigation of the effects of magnetic variations on Inertial/Magnetic orientation sensors. In Proceedings of the 2004 IEEE International Conference on Robotics and Automation, pages 1115-1122, 2004. [cited at p. 16, 20, 58, 85]

[8] Eric R.R. Bachmann. Inertial and Magnetic Tracking of Limb Segment Orientation for Inserting Humans in Synthetic Environments. PhD thesis, Naval Postgraduate School, Monterey, 2000. [cited at p. 16]

[9] Nicole Jacqueline Badke. The effects of changing head position and posture on head tremor in individuals with essential tremor involving the head. $\mathrm{PhD}$ thesis, Queens University, Kingston, Ontario, Canada, 2011. [cited at p. 3]

[10] Fabio Bagala, Valeria Lucia Fuschillo, Lorenzo Chiari, and Angelo Capello. Calibrated 2D Angular Kinematics by Single-Axis Accelerometers: From Inverted Pendulum to N-Link Chain. IEEE Sensors Journal, 12(3):479-486, March 2012. [cited at p. 19]

[11] Neil Barbour and George Schmidt. Inertial Sensor Technology Trends. Sensors Journal, IEEE, 1(4):332-339, December 2001. [cited at p. 8]

[12] Julian Benito-Leon and Elan D. Louis. Essential tremor: emerging views of a common disorder. Nature Clinical Practice Neurology, 2(12):666-678, December 2006. [cited at p. 3]

[13] Eric Berkson, Ryan Aylward, James Zachzewski, Joseph Paradiso, and Thomas Gill. IMU Arrays: The Biomechanics of Baseball Pitching. The 
Orthopaedic Journal at Harvard Medical School, 8:90-94, November 2006. [cited at p. 5]

[14] John E. Bortz. A new mathematical formulation for strapdown inertial navigation. IEEE Transactions of Aerospace and Electronic Systems, AES-7(1):6166, 1971. [cited at p. 10]

[15] Jeroen HM. Bregmann, Ruth E. Mayagoitia, and Ian CH. Smith. A portable system for collecting anatomical joint angles during stair ascent: a comparison with an optical tracking device. Dynamic Medicine, 8(3):1-7, April 2009. [cited at p. 19]

[16] Matthew Brodie, Alan Walmsley, and Wyatt Page. Fusion motion capture: a prototype system using inertial measurement units and GPS for the biomechanical analysis of ski racing. Sports Technology, 1(1):17-28, 2008. [cited at p. 5]

[17] Larry P. Brown, Sandra L. Niehues, Andrew Harran, Patricia Yavorsky, and Paul Hirshman. Upper extremity range of motion and isokinetic strength of the internal and external shoulder rotators in major league baseball players. The American Journal of Sports Medicine, 16(6):577-585, December 1988. [cited at p. 77]

[18] C. Butefisch, H. Hummelsheim, P. Denzler, and K.H. Mauritz. Repetitive training of isolated movement improves the outcome of motor rehabilitation of the centrally paretic hand. J Neurol Sci, 130(1):59-68, May 1995. [cited at p. 4]

[19] Olivier Cappé, Simon Godsill, and Eric Moulines. An overview of existing methods and recent advances in sequential monte carlo. In Proceedings of the IEEE, volume 95, pages 899-924, May 2007. [cited at p. 36, 100] 
REFERENCES

[20] Michael J. Caruso. Applications of magnetic sensors for low cost compass systems. In IEEE Position Location and Navigation Symposium, pages 177184, San Diego, CA, 2000. [cited at p. 20]

[21] Kathy Chung, Mahmoud El-Gohary, Brenna Lobb, and James McNames. Measurement of Levedopa-Induced Dyskinesia with Inertial Sensors. In American Academy of Neurology $61^{\text {st }}$ Annual Meeting. American Academy of Neurology, April 2009. [cited at p. 3]

[22] G. Cooper, L. Sheret, L. McMillan, K. Siliverdis, N. Shan, D. Hodgin, L. Kenny, and D. Howard. Inertial sensor-based knee flexion/extension angle estimation. Journal of Biomechanics, 42(16):2678-2685, December 2009. [cited at p. 19]

[23] John J. Craig. Introduction to Robotics, Mechanics and Control. Electrical and Computer Engineering: Control Engineering. Addison-Wesley, 1989. [cited at p. 21, 26]

[24] Dan Crisan and Arnaud Doucet. A Survey of Convergence Results on Particle Filtering Methods for Practitioners. IEEE Transactions on Signal Processing, 50(3):736-747, March 2002. [cited at p. 99]

[25] Andrea Giovanni Cutti, Cappello Troncossi, Angelo Davalli, and Rinaldo Sacchetti. Soft tissue artefact assessment in humeral axial rotation. Gait Posture, 21:341-349, April 2005. [cited at p. 59]

[26] Hooman Dejnabadi, Brigitte M. Jolles, and Kamiar Aminian. A New Approach to Accurate Measurement of Uniaxial Joint Angles Based on a Combination of Accelerometers and Gyroscopes. IEEE Transactions on Biomedical Engineering, 52(8):1478-1484, August 2005. [cited at p. 19] 
REFERENCES

[27] Hooman Dejnabadi, Brigitte M. Jolles, Emilio Casanova, Pascal Fua, and Kamiar Aminian. Estimation and Visualization of Sagittal Kinematics of Lower Limbs Orientation Using Body-Fixed Sensors. IEEE Transactions on Biomedical Engineering, 53(7):1385-1393, July 2006. [cited at p. 19]

[28] A. Delval, P. Krystkowiak, JL. Blatt, E. Labyt, K. Dujardin, A Destee, P. Derambure, and L. Defebvre. Role of hypokinesia and bradykinesia in gait disturbances in huntington's disease: a biomechanical study. Journal of Neurology, 253(1):73-80, January 2006. [cited at p. 3]

[29] A. Delval, P. Krystkowiak, JL. Blatt, E. Labyt, K. Dujardin, A Destee, P. Derambure, and L. Defebvre. A biomechanical study of gait initiation in huntington's disease. Gait \& Posture, 25(2):279-88, February 2007. [cited at p. 3]

[30] G. Deuschl, R. Wenzelburger, K. Loffler, J. Raethjen, and H. Stilze. Essential tremor and cerebellar dysfunction Clinical and Kinematic analysis of intention tremor. Brain, 123:1568-1580, August 2000. [cited at p. 3]

[31] Arnaud Doucet, Simon Godsill, and Christophe Andrieu. On sequential Monte Carlo sampling methods for Bayesian filtering. Statistics and Computing, 10(3):197-208, Jul 2000. [cited at p. 92]

[32] R. J. Dunnewold, J. I. Hoff, H. C. van Pelt, P. Q. Fredrikze, E. A. Wagemans, and J. J. van Hilten. Ambulatory quantitative assessment of body position, bradykinesia, and hypokinesia in Parkinson's disease. Journal of Clinical Neurophysiology, 15(3):235-242, May 1998. [cited at p. 3]

[33] Mahmoud El-Gohary and James McNames. Shoulder And Elbow Joint Angle Tracking with Inertial Sensors. IEEE Transactions on Biomedical Engineering, 59(9):577-585, July 2012. [cited at p. 46] 
REFERENCES

[34] Mahmoud El-Gohary, James McNames, Kathy Chung, Mateo Aboy, Arash Salarian, and Fay Horak. Continuous At-Home Monitoring of Tremor in Patients with Parkinson's Disease. In Proceedings of Biosignal 2010: Analysis of Biomedical Signals and Images, volume 20, pages 420-424, Brno, Czech Republic, June 2010. Brno University of Technology. [cited at p. 3]

[35] R. J. Elble, M. Brilliant, K. Leffler, and C. Higgins. Quantification of essential tremor in writing and drawing. Movement Disorders, 11(1):70-78, January 2004. [cited at p. 3]

[36] Satoru Emura and Susumu Tachi. Compensation of Time Lag between Actual and Virtual Spaces by Multi-Sensor Integration. In Proceedings of the 1994 IEEE International Conference on Multisensor Fusion and Integration for Intelligent Systems, pages 463-469, 1994. [cited at p. 5]

[37] Stanley Fahn, Richard L. Elton, and UPDRS Development Committee. Unified Parkinsons disease rating scale. In Stanley Fahn, C. David Marsden, Menek Goldstein, and Donald B. Calne, editors, Recent developments in Parkinsons disease, volume 2, pages 153-163, 293-304. Macmillan Healthcare Information, Florham Park, N. J., 1987. [cited at p. 3]

[38] J. Favre, B. M. Jolles, R. Aissaoui, and K. Aminian. Ambulatory Measurement of 3D Knee Joint Angle. Journal of Biomechanics, 41(5):1029-1035, January 2008. [cited at p. 19]

[39] J. Favre, B. M. Jolles, O. Siegrist, and K. Aminian. Quaternion-based fusion of gyroscopes and accelerometers to improve 3D angle measurement. Electronics Letters, 42(11):612-614, May 2006. [cited at p. 19] 
[40] Raul Feliz, Eduardo Zalama, and Jaime Gomez Garcia-Bermejo. Pedestrian tracking using inertial sensors. Journal of Physical Agents, 3(1):35-42, January 2009. [cited at p. 79]

[41] Hilde Feys, Willy De Weerdt, Geert Verbeke, Gail Cox Steck, Chris Capiau, Carlotte Kiekens, Eddy Dejaeger, Gustaaf Van Hoydonck, Guido Vermeersch, and Patrick Cras. Early and Repetitive Stimulation of the Arm Can Substantially Improve the Long-Term Outcome After Stroke: A 5-Year Follow-up Study of a Randomized Trial. Stroke, 35:924-929, April 2004. [cited at p. 4]

[42] A. Forner-Cordero, Jose L. Pons, E. A. Turowska, and A. Schiele. Kinematics and dynamic of wearable robots. In Josè L. Pons, editor, Wearable Robots: Biomechatronic Exoskeletons, chapter 3, pages 47-85. John Wiley \& Sons Ltd, West Sussex, England, 2 edition, 2008. [cited at p. 26, 77]

[43] Donald Fowles and Saadia Greenberg. A Profile of Older Americans: 2011. Technical report, Administration on Agin, U.S. Department of Health and Human Services, July 2011. [cited at p. 4]

[44] E. Foxlin, M. Harrington, and Yury Altshuler. Miniature 6-DOF inertial system for tracking HMDs. In Helmet and Head-Mounted Displays III, volume 3362, pages 21422-21428, 1998. [cited at p. 15]

[45] Eric Foxlin. Inertial head-tracker fusion by a complementary separate-bias Kalman Filter. In Proceedings of Virtual Reality Annual International Symposium, pages 185-194, 1996. [cited at p. 15]

[46] Eric Foxlin. Pedestrian Tracking with Shoe-Mounted Inertial Sensors. IEEE Computer Graphics and Applications In Computer Graphics and Applications, 25(6):38-46, November 2005. [cited at p. 15, 79] 
[47] Daniele Giansanti, Giovanni Maccioni, and Velio Macellari. The Development and Test of a Device for the Reconstruction of 3-D Position and Orientation by Means of a Kinematic Sensor Assembly With Rate Gyroscopes and Accelerometers. IEEE Transactions on Biomedical Engineering, 52(7):12711277, July 2005. [cited at p. 16]

[48] Daniele Giansanti, Velio Macellari, Giovanni Maccioni, and Aurelio Cappozzo. Is it Feasible to Reconstruct Body Segment 3-D Position and Orientation Using Accelerometric Data? IEEE Transactions on Biomedical Engineering, 50(4):476-483, April 2003. [cited at p. 16]

[49] Joseph P. Giuffrida, Alan Lerner, Richard Steiner, and Janis Daly. UpperExtremity Stroke Therapy Task Discrimination Using Motion Sensors and Electromyography. IEEE Transactions on Neural Systems and Rehabilitation Engineering, 16(1):82-90, February 2008. [cited at p. 4]

[50] N.J. Gordon, D.J. Salmond, and A.F.M. Smith. Novel approach to nonlinear/non-Gaussian Bayesian state estimation. In Radar and Signal Processing, IEE Proceedings-F, pages 107-113, April 1993. [cited at p. 36, 100]

[51] Mohinder S. Grewal and Angus P. Andrews. Kalman Filtering: Theory and Practice Using MATLAB. John Wiley \& Sons, Baltimore, MD, 2 edition, 2001. [cited at p. 35]

[52] E. S. Grood and W. J. Suntay. A joint Coordinate System for the Clinical Description of Three-Dimensional Motions: Application to the Knee. ASME, 105:136-142, May 1983. [cited at p. 47]

[53] Kristin Gunderson. The Etiology of Multiple Sclerosis and Correlation of the Distribution of the Disease with Migration and Settlement History of Northern 
Europeans. Master's thesis, Georgia State University, Atlanta, Georgia, July 2007. [cited at p. 4]

[54] Joseph Hamill and Kathleen M. Knutzen. Biomechanical Basis of Human Movement. Lippincott Willimas \& Wilkins, New York, NY, 2 edition, 2003. [cited at p. 23]

[55] Teatsuya Harada, Hiroto Uchino, Taketoshi Mori, and Tomomasa Sato. Portable Absolute Orientation Estimation Device with Wireless Network under Accelerated Situation. In Proceedings of the 2004 IEEE International Conference on Robotics \& Automation, pages 1412-1417, 2004. [cited at p. 20]

[56] W. C. Hayes, J. D. Gran, M. L. Nagurka, J. M. Feldman, and Oatis. C. Leg motion analysis during gait by multiaxial accelerometry: theoretical foundations and preliminary validations. Journal of Biomechanical Engineering, 105(3):283-289, August 1983. [cited at p. 4]

[57] Ernst A. Heinz, Kai Kunze, Matthias Gruber, David Bannach, and Paul Lukowicz. Using Wearable Sensors for Real-time Recognition Tasks in Games of Martial Arts - An Initial Experiment. In ISWC '05: Proceedings of IEEE CIG'06, 2006. [cited at p. 5]

[58] T. Hermann, O. Honer, and H. and Ritter. Acoumotion - an interactive sonification system for acoustic motion control. In Gesture in Human-Computer Interaction and Simulation: 6th International Gesture Workshop, pages 312323, 2006. [cited at p. 7]

[59] Todd Hester, Richard Hughes, Delsey Sherril, and Bethany Knorr. Using Wearable Sensors to Measure Abilities following Stroke. In Proceedings of 
the International Workshop on Wearable and Implantable Body Sensor Networks, 2006. [cited at p. 4]

[60] Jorrit I. Hoff, Bob J. van Hilten, and Raymund A. C. Roos. A review of the assessment of dyskinesias. Movement Disorders, 14(5):737-743, Sep 1999. [cited at p. 2]

[61] X. Huang, JM. Mahoney, MM. Lewis, Du. Guangwei, SJ. Piazza, and JP. Cusumano. Both coordination and symmetry of arm swing are reduced in Parkinson's disease. Gait Posture, 35(3):373-377, March 2012. [cited at p. 3]

[62] Sara Imarisio, Jenny Carmichael, Viktor Korolchuck, Chien-Wen Chen, Shinji Saiki, Claudia Rose, Gauri Krishna, Janet Davies, Evangelia Ttofi, Benjamin Underwood, and David Rubinsztein. Huntington's disease: from pathology and genetics to potential therapies. Biochemical Journal, 1(412):191-209, June 2008. [cited at p. 3]

[63] S. J. Julier and J. K. Uhlmann. Unscented filtering and nonlinear estimation. In Processings of The IEEE, volume 92, pages 401-422, March 2004. [cited at p. 22, 35, 38]

[64] T. Kailath, A. H. Sayed, and B. Hassibi. Linear Estimation. Prentice Hall, 2000. [cited at p. 20]

[65] R. E. Kalman. A new approach to linear filtering and prediction problems. Transactions of the ASME - Journal of Basic Engineering, 82:35-45, 1960. [cited at p. 35]

[66] Steven M. Kay. Fundamentals of Statistical Signal Processing, Estimation Theory. Prentice Hall, 1993. [cited at p. 35] 
REFERENCES

[67] Noël L. Keijsers, Martin W. Horstink, and Stan C. Gielen. Online monitoring of dyskinesia in patients with Parkinson's disease. IEEE Engineering in Medicine and Biology Magazine, 22(3):96-103, 2003. [cited at p. 46]

[68] Kevin King, S.W. Yoon, N.C. Perkins, and K. Najafi. Wireless MEMS inertial sensor system for golf swing dynamics. Sensors and Actuators A: Physical, 141(2):619-630, February 2008. [cited at p. 5]

[69] Thomas Klockgether. Parkinson's disease: clinical aspects. Cell and Tissue Research, 318(1):115-120, October 2004. [cited at p. 2]

[70] Edgar Kraft. A Quaternion-based Unscented Kalman Filter for Orientation Tracking. In Proceedings of the IEEE 6th International Conference information fusion, pages 47-54, 2003. [cited at p. 21, 104]

[71] Leslie A. Kurtz. Digital Actors and Copyright - From 'The Polar Express' to 'Simone'. Santa Clara Computer and High Technology Law Journal, , 21:783, 2005. [cited at p. xi, 7]

[72] Michael Tomasz Lapinski. A Wearable, Wireless Sensor System for Sports Medicine. PhD thesis, School of Architecture and Planning, Massachusetts Institute of Technology, 2008. [cited at p. 5]

[73] Kun Liu, Tao Liu, Kyoko Shibata, Yoshio Inoue, and Rencheng Zheng. Novel approach to ambulatory assessment of human segmental orientation on a wearable sensor system. Journal of Biomechanics, 42(16):2747-2752, September 2009. [cited at p. 19]

[74] Henk J. Luinge. Inertial Sensing of Human Motion. PhD thesis, Univeristy of Twente, Enschede, The Netherlands, December 2002. [cited at p. 16] 
REFERENCES

[75] Henk J. Luinge and Peter H. Veltink. Inclination Measurement of Human Movement Using a 3-D Accelerometer With Autocalibration. IEEE Transactions on Neural Systems and Rehabilitation Engineering, 12(1):112-121, March 2004. [cited at p. 16]

[76] Henk J. Luinge and Peter H. Veltink. Measuring orientation of human body segments using miniature gyroscopes and accelerometers. Medical \& biological engineering \& computing, 43(2):273-282, Mar 2005. [cited at p. 16]

[77] Henk J. Luinge, Peter H. Veltink, and Chris T. M. Baten. Estimating orientation with gyroscopes and accelerometers. Technology and Health Care, 7(6):455-459, January 1999. [cited at p. 16]

[78] Henk J. Luinge, Peter H. Veltink, and Chris T. M. Baten. Ambulatory Measurement of Arm Orientation. Journal of Biomechanics, 40:78-85, 2007. [cited at p. 19]

[79] A. J. Manson, P. Brown, J. D. O’Sullivan, P. Asselman, D. Buckwell, and A. J. Lees. An ambulatory dyskinesia monitor. Journal of Neurology, Neurosurgery, and Psychiatry, 68(2):196-201, Feb 2000. [cited at p. 2]

[80] Ruth E. Mayagoitia, Anand V. Nene, and Peter H. Veltink. Accelerometer and rate gyroscope measurement of kinematics: an inexpensive alternative to optical motion analysis systems. Journal of Biomechanics, 35(4):537-542, Apr 2002. [cited at p. 15]

[81] Leonard A. McGee and Stanley F. Schmidt. Computational techniques in Kalman filtering. Memorandum 86847, National Aeronautics and Space Administration, November 1985. [cited at p. 35] 
REFERENCES

[82] James McNames, Mahmoud El-Gohary, and Sean Pearson. Joint angle tracking with inertial sensors. U.S. Patent 2009/0204031 A1, August 2009. [cited at p. 58]

[83] Rudolph van der Merwe, Arnaud Doucet, Nando De Freitas, and Eric Wan. The Unscented Particle Filter. In NIPS, pages 584-590, 2000. [cited at p. 38]

[84] Kim Mitchell, Scott Banks, David Morgan, and Hiroyuki Sugaya. Shoulder motions during the golf swing in male amateur golfers. Journal of Orthopaedic \& Sports Physical Therapy, 33(4):196-203, April 2003. [cited at p. 5]

[85] P. Munter, E. Garrett, M. J. Klag, and J. Coresh. Trends in stroke prevalence between 1973 and 1991 in the US population 25 to 74 years of age. Stroke, 33(5):1209-1213, 2002. [cited at p. 4]

[86] C. Musso, N. Oudjane, and F. Legland. Improving regularized particle filters. In Sequential Monte Carlo Methods in Practice, pages 247-271, 2001. [cited at p. 100]

[87] Bijan Najafi, Kamiar Aminian, Anisoara Paraschiv-Ionescu, François Loew, and Christopher Bula. Ambulatory System for Human Motion Analysis Using a Kinematic Sensor: Monitoring of Daily Physical Activity in the Elderly. IEEE Transaction on Biomedical Engineering, 50(6):711-723, June 2003. [cited at p. 4]

[88] John G. Nutt and G. Frederick Wooten. Clinical practice. diagnosis and initial management of Parkinson's disease. New England Journal of Medicine, 353(10):1021-1027, Sep 2005. [cited at p. 2]

[89] James F. O’Brien, Robert E. Bodenheimer, Gabriel J. Brostow, and Jessica K. Hodgins. Automatic Joint Parameter Estimation from Magnetic Motion Cap- 
REFERENCES

ture Data. In Proceedings of Graphics Interface, pages 53-60, May 2000. [cited at p. 6]

[90] M. Pitt and N. Shephard. Filtering via simulation: Auxiliary particle filters. Journal of the American Statistical Association, 94(446):590-599, 1999. [cited at p. 100]

[91] Frederick H. Raab, Ernest B. Blood, Terry O. Steiner, and Herbert R. Jones. Magnetic Position and Orientation Tracking System. In IEEE Transactions on Aerospace and Electronics Systems, volume AES-15, pages 709-718, 1979. [cited at p. 6]

[92] Shobha S. Rao, Laura A. Hofman, and Amer Shakil. Parkinson's disease: diagnosis and treatment. The American Academy of Family Physicians, 74(12):2046-2054, December 2006. [cited at p. 2]

[93] Olivier Rascol, David Brooks, Amos Korczyn, Peter De Deyn, Carl Clarke, and Anthony Lang. A five-Year Study of The Incidence of Dyskinesia in Patients with Early Parkinson's Disease Who were Treated with Ropinirole or Levodopa. The New England Journal of Medicine, pages 1484-1491, May 2000. [cited at p. 2]

[94] Ian B. Rhodes. A tutorial introduction to estimation and filtering. IEEE Transactions on Automatic Control, AC-16(6):688-706, Decemeber 1971. [cited at p. 87]

[95] Branko Ristic, Sanjeev Arulampalam, and Neil Gordon. Beyond the Kalman Filter. Artech House, 2004. [cited at p. 34]

[96] Daniel Roetenberg. Inertial and Magnetic Sensing of Human Motion. PhD thesis, University of Twente, Enschede, The Netherlands, 2006. [cited at p. 15, 85] 
REFERENCES

[97] Daniel Roetenberg, Henk J. Luinge, Chris T. M. Baten, and Peter H. Veltink. Compensation of Magnetic Disturbances Improves Inertial and Magnetic Sensing of Human Body Segment Orientation. IEEE Transactions on Neural Systems and Rehabilitation Engineering, 13(3):395-405, September 2005. [cited at p. 17]

[98] Daniel Roetenberg, Per J. Slycke, and Peter H. Veltink. Ambulatory Position and Orientation Tracking Fusing Magnetic and Inertial Sensing. IEEE Transactions on Biomedical Engineering, 54(5):883-890, May 2007. [cited at p. 17]

[99] Douglas P. Romilly, Carolyn Anglin, Raymond G. Gosine, and Silvia U. Raschke. A Functional Task Analysis and Motion Simulation for the Development of a Powered Upper-Limb Orthosis. IEEE Transactions on Rehabilitation Engineering, 2(3):119-129, September 1994. [cited at p. 4]

[100] Li X. Rong and Vesselin P. Jilkov. A Survey of Maneuvering Target Tracking: Approximation Techniques for Nonlinear Filtering. In SPIE Conference on Signal and Data Processing of Small Targets, pages 537-550, April 2004. [cited at p. 87]

[101] E. Roux, S. Bouilland, A. Godillon-Maquinghen, and D. Bouttens. Evaluation of the global optimization method within the upper limb kinematics analysis. Journal of Biomechanics, 35(9):1279-1283, September 2002. [cited at p. 6]

[102] Angelo M. Sabatini. Assessment of walking features from foot inertial sensing. IEEE Transactions on Biomedical Engineering, 52(3):486-494, March 2005. [cited at p. 17] 
REFERENCES

[103] Angelo M. Sabatini. Quaternion-Based Extended Kalman Filter for Determining Orientation by Inertial and Magnetic Sensing. IEEE Transactions on Biomedical Engineering, 53(7):1346-1356, July 2006. [cited at p. 18]

[104] Carsten Saft, Jurgen Andrich, Nina-Marie Meisel, Horst Przuntek, and Thomas Muller. Assessment of complex movements reflects dysfunction in Huntington's disease. J Neurol, 250:1469-1474, December 2003. [cited at p. 3]

[105] A. Salarian, H. Russmann, F. J. G. Vingerhoets, C. Dehollain, Y. Blanc, P. R. Burkhard, and K. Aminian. Gait assessment in parkinson's disease: Toward an ambulatory system for long-term monitoring. IEEE Transactions on Biomedical Engineering, 51(8):1434-1443, August 2004. [cited at p. 3]

[106] A. Salarian, H. Russmann, F.J.G. Vingerhoets, P.R. Burkhard, Y. Blanc, C. Dehollain, and K. Aminian. An ambulatory system to quantify bradykinesia and tremor in Parkinson's disease. In IEEE EMBS Special Topic Conference on Information Technology Applications in Biomedicine, pages 35-38, 24-26 April 2003. [cited at p. 3]

[107] Ali Samii, John G. Nutt, and Bruce R. Ransom. Parkinson's disease. Lancet, 363(9423):1783-1793, May 2004. [cited at p. 2]

[108] Martin Schepers, H.F. Koopman, and Peter H. Veltink. Ambulatory Assessment of Ankle and Foot Dynamics. IEEE Transactions on Biomedical Engineering, 54(5):895-901, July 2007. [cited at p. 79]

[109] Dan Simon. Kalman filtering with state constraints: a survey of linear and nonlinear algorithms. Control Theory \& Application, 4(8):1303-1318, August 2010. [cited at p. 77] 
REFERENCES

[110] Isaac Skog, Peter Händel, John-Olof Nilsson, and Jouni Rantakokko. ZeroVelocity Detection - An Algorithm Evaluation. IEEE Transaction on Biomedical Engineering, 57(11):2657-2666, November 2010. [cited at p. 79]

[111] Richard G. Snyder, Don B. Chaffin, and Rodney K. Schutz. Link System Of The Human Torso. Technical report, Michigan University Ann Arbor Highway Safety Research Institute, July 1972. [cited at p. 77]

[112] R. Spain, R. St. George, A. Salarian, M. Mancini, J. Wagner, F. Horak, and D. Bourdette. Body-worn motion sensors detect balance and gait deficits in people with multiple sclerosis who have normal walking speed. Gait \&Posture, 35(4):573-578, April 2012. [cited at p. 4]

[113] Ross Stirling, Jussi Collin, Ken Fyfe, and Gerard Lachapelle. An innovative shoe-mounted pedestrian navigation system. In European Navigation Conference (GNSS), April 2003. [cited at p. 61]

[114] K. Tong and MH. Granat. A practical gait analysis system using gyroscopes. Journal of Biomechancis, 21(2):87-94, 1999. [cited at p. 15]

[115] Peter H. Veltink, Erwin G.O. Engberink, Bob J. Van Hilten, Rob Dunnewold, and Cathrien Jacobi. Towards a new method for kinematic quantification of bradykinesia in patients with Parkinson's disease using triaxial accelerometry. In IEEE 17th Annual Conference Engineering in Medicine and Biology Society, volume 2, pages 1303-1304, September 1995. [cited at p. 3]

[116] Eric A. Wan and Rudolph van der Merwe. The unscented Kalman filter. In Simon Haykin, editor, Kalman Filtering and Neural Networks. John Wiley \& Sons, 2001. [cited at p. 20, 36] 
REFERENCES

[117] Greg Welch and Eric Foxlin. Motion Tracking: No Silver Bullet, but a Respectable Arsenal. IEEE Computer Graphics and Applications, 22(6):24-38, Nov-Dec 2002. [cited at p. 5, 6]

[118] Sherry L. Werner, Tricia A. Murray, Richard J. Hawkins, and Thomas J. Gill. Relationship between throwing mechanics and elbow valgus in professional baseball pitchers. Journal of Elbow Surgery, pages 151-155, March/April 2002. [cited at p. 5]

[119] Antoon. Th. M. Willemsen, Carlo Frigo, and Herman B. K. Boom. Lower extremity angle measurement with accelerometers - error and sensitivity analysis. IEEE Transactions on Biomedical Engineering, 38(12):1186-1193, 1991. [cited at p. 15]

[120] Antoon Th. M. Willemsen, J. A. Van Alste, and Herman B. K. Boom. Realtime gait assessment utilizing a new way of accelerometery. Journal of Biomechanics, 23(8):859-863, 1990. [cited at p. 15]

[121] R. Williamson and B. J. Andrews. Detecting absolute human knee angle and angular velocity using accelerometers and rate gyroscopes. Medical and Biological Engineering and Computing, 39:1-9, May 2001. [cited at p. 15]

[122] Oliver Woodman. An introduction to inertial navigation. Technical report, Computer Laboratory, University of Cambridge, UK, August 2007. [cited at p. 65]

[123] Rick W. Wright, Karen Steger-May, Brett L. Wasserlauf, Mark E. O’Neal, Barry W. Weinberg, and George A. Paletta. Elbow Range of Motion in Professional Baseball Pitchers. American Journal of Sports Medicine, 34(2):190193, February 2006. [cited at p. 5] 
[124] S. Yabukami, H. Kikuchi, M. Yamaguchi, K. I. Arai, K. Takahashi, A. Itagaki, and $\mathrm{N}$ Wako. Motion capture system of magnetic markers using three-axial magnetic field sensor. IEEE Transactions on Magnetics, 36(5):3646-3648, Septemeber 2000. [cited at p. 7]

[125] Soo-Yeong Yi and Byoung-Wook Choi. Autonomous Navigation of Indoor Mobile Robots Using a Global Ultrasonic System. Robotica, 22:369-374, August 2004. [cited at p. 7]

[126] Xiaoping Yun, Conrado Aparicio, Eric R. Bachmann, and Robert B. McGhee. Implementation and Experimental Results of a Quaternion-Based Kalman Filter for Human Body Motion Tracking. In Proceedings of the 2005 IEEE International Conference on Robotics and Automation, pages 317-322, 2005. [cited at p. 18]

[127] Xiaoping Yun and Eric R. Bachmann. Design, Implementation, and Experimental Results of a Quaternion-Based Kalman Filter for Human Body Motion Tracking. IEEE Transaction on Robotics, 22:1217-1227, Decemeber 2006. [cited at p. 18]

[128] Xiaoping Yun, Eric R. Bachmann, and Robert B. McGhee. A Simplified Quaternion-Based Algorithm for Orientation Estimation From Earth Gravity and Magnetic Field Measurements. IEEE Transactions on Instrumentation and Measurement, 57(3):638-650, March 2008. [cited at p. 18]

[129] Huiyu Zhou and Huosheng Hu. Inertial motion tracking of human arm movements in stroke rehabilitation. In Proceedings of the IEEE International Conference on Mechatronics and Automation, pages 1306-1311, 2005. [cited at p. 18] 
[130] Huiyu Zhou and Huosheng Hu. Upper limb motion estimation from inertial measurements. International Journal of Information Technology, 13(1):1-14, 2007. [cited at p. 18]

[131] Huiyu Zhou and Huosheng Hu. Human motion tracking for rehabilitationa survey. Biomedical Signal Processing and Control, 3:1-18, January 2008. [cited at p. 2]

[132] Huiyu Zhou and Huosheng Hu. Reducing drifts in the inertial measurements of wrist and elbow positions. IEEE Transactions on Instrumentation and Measurement, 59(3):575-585, 2010. [cited at p. 18]

[133] Huiyu Zhou, Huosheng Hu, and Yaqin Tao. Inertial measurements of upper limb motion. Medical \& biological engineering \& computing, 44:479-487, 2006. [cited at p. 18]

[134] D.G.M. Zwartjes, T. Heida, J.P.P. van Vugt, J.A.G. Geelen, and P.H. Veltink. Ambulatory Monitoring of Activities and Motor Symptoms in Parkinson's Disease. IEEE Transactions on Biomedical Engineering, 57(11):2778-2786, November 2010. [cited at p. 3] 